University of Navarra

\title{
COMPETITION IN THE SUPPLY OPTION MARKET
}

\author{
Víctor Martínez de Albéniz ${ }^{1}$ \\ David Simchi-Levi
}

${ }^{1}$ Professor, Operations Management and Technology, IESE

${ }^{2}$ Professor, Operations Research Center, MIT 


\title{
Competition in the Supply Option Market ${ }^{1}$
}

\author{
Submitted: July 17, 2006. Revised: July 19, 2007.
}

Victor Martínez-de-Albéniz ${ }^{2}$ and David Simchi-Levi ${ }^{3}$

\begin{abstract}
This paper develops a multi-attribute competition model for procurement of short life cycle products. In such an environment, the buyer installs dedicated production capacity at the suppliers before the demand is realized. Final production orders are decided after demand materializes. Of course, the buyer is reluctant to bear all the capacity and inventory risk, and thus signs flexible contracts with several suppliers. We model the suppliers' offers as option contracts, where each supplier charges a reservation price per unit of capacity, and an execution price per unit of delivered supply. These two parameters illustrate the trade-off between total price and flexibility of the contract, and are both important to the buyer. We model the interaction between the suppliers and the buyer as a game in which the suppliers are the leaders and the buyer is the follower. Specifically, suppliers compete to provide supply capacity to the buyer and the buyer optimizes its expected profit by selecting one or more suppliers. We characterize the suppliers' equilibria in pure strategies for a class of customer demand distributions. In particular, we show that this type of interaction gives rise to cluster competition. That is, in equilibrium, suppliers tend to be clustered in small groups of two or three suppliers each, such that within the same group all suppliers use similar technologies and offer the same type of contract. Finally, we show that in equilibrium, the supply chain inefficiencies, i.e., the loss of profit due to competition, are in general at most $25 \%$ of the profit of a centralized supply chain, for a wide class of demand distributions.
\end{abstract}

\section{Introduction}

The introduction of new products is usually associated with uncertain sales forecasts. When the product life-cycle is short, firms usually have limited opportunities to delay decisions until the time accurate forecasts are available. For instance, it would be ideal to delay decisions about production quantities until the beginning of the selling season, when initial sales have been observed and thus sales expectations are more robust. However, if production lead times are long, this is obviously not possible, and thus firms must take capacity and inventory risks at product launch. In industries like electronics or fashion retailing, managing these risks appropriately is critical for the long-term survival of firms.

One way to reduce the financial impact of these risks is by adjusting supply costs with sales realizations. This is done through flexible contracts which allow scaling up production volume and costs with sales volume and revenues. The typical example of a flexible contract is the option contract. This contract is characterized by two parameters, a capacity reservation fee and an execution fee. For each unit of capacity installed by the supplier, the buyer pays in advance the reservation fee. When demand is realized, the buyer decides how many units to order, and for each

\footnotetext{
${ }^{1}$ Research supported in part by the Center of eBusiness at MIT, ONR Contracts N00014-95-1-0232 and N00014-01-1-0146, NSF Contracts DMI-9732795, DMI-0085683 and DMI-0245352.

${ }^{2}$ IESE Business School, Av. Pearson 21, 08034 Barcelona, Spain.

${ }^{3}$ Operations Research Center, MIT, 77 Mass. Ave., Cambridge MA 02139, USA.
} 
unit pays the execution fee. Thus, if demand is smaller than expected, the buyer pays execution fee only on realized demand, and not on the entire capacity installed.

Contracts similar to option contracts are common in industries such as textiles, plastics or semiconductors manufacturing. In some cases, such contracts are disguised under the name of buy-back contracts, which are equivalent to an option, as in newspaper or book distribution. Specifically, in a buy-back contract, the cost of the component and the amount of the refund for returned items are specified; this corresponds to an option with reservation price equal to the total cost minus the refund, and an execution price equal to the refund. It is well known, see Pasternack [15], that buy-back contracts can be beneficial to both the buyer and the supplier, since they can coordinate the supply chain.

As shown by Martínez-de-Albéniz and Simchi-Levi [13], one way for a buyer to better manage its risks is to simultaneously sign several option contracts with a number of suppliers. This allows the buyer to take advantage of the relative cost and flexibility of the different contracts. For instance, it can sign a low-cost contract with little flexibility for the most certain portion of the demand, and sign a more expensive but flexible contract for the more volatile part of sales. An illustration of this strategy can be found in apparel retailing, where a retailer may place a large order in China, at a low price, and at the same time, reserve some capacity locally and have the option to scale up production if demand is high.

Evidently, this purchasing strategy can force changes in the way suppliers compete in the marketplace. Clearly, flexibility and price are the two attributes that the buyer cares about, and suppliers should take note of it. The objective of this paper is precisely to analyze the suppliers' pricing strategy when they are competing through price and flexibility. Specifically, our objective is to characterize option contracts offered by competing suppliers in equilibrium.

For this purpose, we focus on products with short life cycles and consider a single period model with many suppliers and a single buyer purchasing a single component. The sequence of events is as follows. First, each supplier offers an option contract to the buyer, with a given reservation and execution fee. After receiving all the competing bids, the buyer reserves capacity with some or all the suppliers. Finally, after demand is realized, the buyer requests deliveries from each supplier, up to the installed capacity.

Of course, a supplier needs to take into account the competitors' bids when offering its preferred contract. A given supplier can thus undertake two main actions to become more competitive: either lower the reservation price or lower the execution price. The trade-off is clear. A supplier that charges mainly a reservation fee (and a small execution fee) competes on price but not flexibility. On the other hand, a supplier that charges mainly an execution fee (and a small reservation fee) typically emphasizes flexibility and not price.

Evidently, the suppliers bids depend on their cost structure. We assume that there are two types of costs for each supplier. 
- A reservation cost is associated with setting up the line and making preparations for production. We assume that this reservation cost has a linear or per-unit cost structure, corresponding to the acquisition of special machinery or specialized labor, that scales up with the level of capacity requested by the buyer.

- An execution cost is then paid when the supplier finalizes production and ships the components after it receives the firm and final order from the buyer. This additional work has also a per-unit cost structure, corresponding to labor, material and logistics cost.

Different suppliers may have different costs for reserving capacity and delivering supply, depending on the type of technology (machinery) and their geographical location (labor, transportation). In addition, the cost structure of each firm may also be determined by its production strategy: a company that buys dedicated machines early on incurs most of the cost as a reservation cost; a company that leases these same machines later on has the ability to pass the corresponding cost as execution cost.

The supplier cost model is consistent with situations where the capacity installed under contract is dedicated to the buyer, and not shared with other firms. For instance, we are familiar with a large Taiwanese contract manufacturer that, upon signing a supply contract with a buyer, typically sets up a dedicated line for that buyer, in advance of the production season. The reservation cost for the dedicated line clearly increases with the capacity level. After demand is realized, the buyer, a PC manufacturer in this case, decides a final order quantity, up to the capacity, and this is produced and shipped by the contract manufacturer. The cost associated with production and shipping is the execution cost defined earlier. As a matter of fact, this PC manufacturer uses another supplier for the same component, with presumably a different cost structure.

Our objective in this paper is to understand how these suppliers compete. The model captures the multiple cost dimensions of the suppliers, i.e., reservation and execution costs, who compete and differentiate on their prices, i.e., reservation and execution prices.

The paper describes the market equilibrium outcomes of the suppliers' option pricing game. We characterize the suppliers' equilibria in pure strategies for a class of customer demand distributions. Interestingly, this model is an extension of the Bertrand price competition model to two dimensions. An important result in one dimension is that, in equilibrium, there is a unique supplier, the least costly supplier, that captures all the orders at a market price that is between its cost and the cost of the second most competitive supplier. We show that this is not the case when two attributes are important to the buyer. Indeed, we demonstrate that in equilibrium, a variety of suppliers coexists, and these suppliers offer different prices. We call this cluster competition, since suppliers tend to cluster in small groups of two or three suppliers each, such that within the same group all suppliers use similar technologies and offer the same type of contract.

Intuitively, we have shown that the best strategy of each supplier is to set a price very similar to 
some other supplier, while making sure that the share of capacity "stolen" from that supplier yields profit. Thus, the supplier does not simply undercut this other supplier, but instead skims carefully the type of capacity (e.g., with higher or lower probability of execution) that it wants to capture.

In addition, we show that in equilibrium, the supply chain inefficiencies, i.e., the loss of profit due to competition, are in general at most $25 \%$ of the first-best, i.e., the profit of a centralized supply chain, for a wide class of demand distributions. Finally, supplier competition through option contracts is particularly attractive to the buyer, since it may allocate more profit to the buyer than an Expected Vickrey-Clark-Groves (EVCG) mechanism, see Schummer and Vohra [17].

We start by reviewing in Section 2 the different streams of literature relevant to our research and present the model in Section 3. We then analyze the buyer's behavior in Section 4 and the suppliers' strategies in Section 5. This leads, in Section 6, to the study of the equilibria of the negotiation process. Finally, we conclude with managerial insights in Section 7.

\section{Literature review}

Our starting point for this research is the recent paper by Martínez-de-Albéniz and Simchi-Levi [13]. In their work, they develop a multi-period framework in which buyers optimize their purchasing strategy by carefully balancing price and flexibility. In particular, in their single period version, they provide a closed form expression for the amounts of option capacities that a buyer purchases from a pool of suppliers. We apply this result in the analysis of the behavior of the suppliers in such a setting, where competition is carried through two dimensions: price and flexibility, or equivalently reservation and execution prices.

We relate this research to the literature on supply contracts; for a review see Cachon [4] or Lariviere [11]. In particular, some papers study option contracts, e.g., Barnes-Schuster et al. [1] or Eppen and Iyer [7]. More relevant to our model are papers that analyze the behavior of suppliers in offering options to a buyer, the prelude to introducing competition between suppliers. The existing literature usually models a sequential game à la Stackelberg, where a single buyer is the follower and a single supplier is the leader. Typically, competition in such models is introduced by a spot market. This spot market is the buyer's sourcing alternative and a potential client for the supplier. The focus is on finding conditions for which both players are willing to sign a contract and determining option prices as the outcome of the negotiation process. Our paper moves from the traditional models of competition through dual sourcing, i.e., single supplier offering an option contract versus spot market, to a model of pure competition between suppliers offering different types of options.

The first publication in this stream of literature is by Wu et al. [22]. Motivated by electricity markets, they derive option prices as a function of the costs of the system, the spot price distribution and the buyer's utility. Later, Spinler et al. [18] and Golovachkina and Bradley [10] analyze similar models. Building on this, a multi-sourcing version is presented in $\mathrm{Wu}$ and Kleindorfer [20]. In 
this work, suppliers are characterized by an execution unit cost and a total capacity, and offer option contracts to the buyer. Wu and Kleindorfer derive Bertrand-like results, where competitive suppliers contract with the buyer up to their available capacity. Wu et al. [21] expand this model by proposing a capacity investment game between the suppliers, where, after installing capacity, the short-run price competition presented in $\mathrm{Wu}$ and Kleindorfer [20] takes place.

Interestingly, Wu and Kleindorfer [20] assume the same cost structure as ours, but suppliers make capacity investments before price competition, there is a random spot price and the buyer has a deterministic utility (which implies a deterministic demand function); in our model, on the other hand, capacity investments follow pricing decisions, there is no spot market but a random demand, and suppliers are uncapacitated. These differences lead to significantly different equilibrium characteristics. Specifically, Wu and Kleindorfer show that the buyer follows a greedy contracting rule, i.e., purchases capacity from the suppliers with the lowest overall price, up to capacity. Thus, the suppliers' equilibrium is such that all suppliers active in the contracting market offer an identical overall price. Potentially, if all suppliers have infinite capacity, Wu and Kleindorfer imply that a single supplier will be active in the contract market. Thus, deterministic utility leads to single sourcing, and multi-sourcing comes from having capacitated suppliers. In comparison, uncertainty in demand in our model leads to multi-sourcing, since multi-sourcing helps manage demand uncertainty in a cheaper way than single sourcing.

Another related stream of the literature concentrates on analyzing multi-attribute auctions. This research is quite recent and follows the development of online auctions in B2B markets. Typically, the objective is to design the auction mechanism so as to reach an optimal outcome. An optimal outcome may be defined as social efficiency or profit maximization from the auctioneer's point of view (e.g., Myerson [14] in a one-dimensional auction). Usually, there is uncertainty in the suppliers' cost structure, and hence the design of the auction is done using probabilistic distributions of costs. In our paper, however, the costs are assumed to be deterministic and known to all the players; hence, our modeling approach is very different than the approach in this group of papers.

In this line of research, various authors have studied the winner determination problem, where a single supplier is awarded all the orders. This differs from our formulation where all the suppliers may potentially be selected for part of the procurement. For instance, Beil and Wein [2], following Che [5], present a multi-attribute Request For Quotation (RFQ) process where the buyer declares a scoring rule and chooses a winner among many suppliers, the one that obtains the highest score for the declared rule.

In a different direction, some research has been done on mechanism design where many bidders can be awarded orders at the same time. For instance, Schummer and Vohra [17] analyze a class of two-dimensional option auction mechanisms for a set of suppliers confronted with a single buyer. Their formulation is similar to our model but focuses on designing an efficient procurement mechanism where the suppliers have the incentive to truthfully reveal their costs. Because suppli- 
ers submit their true costs, their paper does not directly address competition between suppliers. In comparison, we analyze a supplier competition model where suppliers are paid what they bid, under complete information.

\section{Assumptions and Notation}

Consider a single buyer purchasing a component that is used in the manufacturing of the final product. This component may be obtained from a variety of suppliers. We make two assumptions regarding selling price and demand observed by the buyer.

Assumption 1 The buyer sells to end customers at a given unit price $p$ fixed in advance.

Assumption 2 The total customer demand $D$ follows a distribution defined over an interval $[\underline{d}, \bar{d}] \subset$ $[0, \infty]$. The c.d.f. of the demand $F(\cdot)$ is strictly increasing in $[\underline{d}, \bar{d}]$. We assume that $F(\cdot)$ is a continuous and differentiable function over $(\underline{d}, \bar{d})$. Define $f(\cdot)=F^{\prime}(\cdot)$ and $\bar{F}(\cdot)=1-F(\cdot)$.

The buyer's objective is to maximize expected profit by optimally selecting the amount of capacity to reserve from each supplier.

We denote by $N$ the number of suppliers in the market. The suppliers' cost structure is assumed to consist of two parts. Each supplier incurs a fixed unit cost for reserving capacity, $f_{i}, i=1, \ldots, N$ that can be seen as the unit cost of installing dedicated capacity in advance of production. In addition, the suppliers pay a unit cost, $c_{i}, i=1, \ldots, N$, for each unit executed by the buyer, which corresponds to the cost of finalizing the component plus transportation. These costs differ from supplier to supplier and may be explained by the use of different technologies or management practices. Without loss of generality, we assume that $c_{1} \leq \ldots \leq c_{N}$.

Each supplier offers an option contract to the buyer. Such a contract is defined by two parameters, $v \geq 0$, the reservation price, and $w \geq 0$, the execution price. These values are determined by the supplier based on its cost structure as well as on whether the supplier emphasizes price or flexibility. Specifically, supplier $i, i=1, \ldots, N$, takes position in the market by offering options at a reservation price $v_{i}$ and an execution price $w_{i}$.

Given the suppliers' offerings, the buyer specifies the amount of capacity to reserve with each supplier ${ }^{4}$. At the time the buyer executes a contract with a supplier, it can purchase any amount $q$, where $q$ is no more than the reserved capacity with that supplier. Thus, the profit of supplier $i$, $i=1, \ldots, N$, is $\left(v_{i}-f_{i}\right) x_{i}+\left(w_{i}-c_{i}\right) q_{i}$ when a buyer reserves $x_{i}$ units of capacity and executes $q_{i}$ units, $0 \leq q_{i} \leq x_{i}$. The objective of the suppliers is to maximize their expected profit by selecting $\left(w_{i}, v_{i}\right)$ optimally.

\footnotetext{
${ }^{4}$ It can be shown that the suppliers have no incentive to build more than the amount specified in the contracts, since any additional capacity, for which no reservation fee is received, would fetch negative profit.
} 
We analyze a two-stage model. In the first stage all the suppliers submit bids that are defined by $\left(w_{i}, v_{i}\right), i=1, \ldots, N$. At the same time, and based on these bids, the buyer decides on the amount of capacity to reserve with each supplier. In the second period, demand is realized and the buyer decides the amount to execute from each contract. If total capacity is not enough, unsatisfied demand is lost.

This is a game in which the suppliers are first-movers and the buyer reacts myopically to the suppliers' bids. Thus, there are multiple players that compete knowing the reaction of the buyer. Suppliers have complete visibility on the buyer's decision making process, as well as on the demand distribution. Therefore, given any $N$ pairs $\left(w_{i}, v_{i}\right), i=1, \ldots, N$, each supplier can figure out the amount of capacity that the buyer would reserve with each individual supplier as well as the distribution of the amount of supply executed (requested) by the buyer.

We assume that the suppliers submit sealed bids simultaneously. Thus, this is a one-shot game. We are interested in determining the equilibria of this game in pure strategies, i.e., the $N$-uples $\left(w_{i}, v_{i}\right), i=1, \ldots, N$, where no supplier has an incentive to unilaterally change its bid.

Information-wise, we assume that the cost parameters of the suppliers are known to each other. Indeed, in practice, most firms have a rather precise idea on the type of technology used by each one of their competitors. This is a strong assumption, which is found as well in the asymmetric Bertrand or Hotelling models, for instance.

\section{The Buyer's Procurement Strategy}

Martínez-de-Albéniz and Simchi-Levi [13] present a general framework for supply contracts in which portfolios of options can be analyzed and optimized. In this section, we review the framework in the context of a single period environment.

Consider a buyer facing $N$ different options with terms $\left(w_{i}, v_{i}\right), i=1, \ldots, N$. Martínezde-Albéniz and Simchi-Levi show that the buyer's expected profit is concave in the quantities $\left(x_{1}, \ldots, x_{N}\right)$ purchased. Without loss of generality, assume that $w_{1} \leq \ldots \leq w_{N} \leq p$. Define $w_{N+1}=p, v_{N+1}=0$ and $q_{N+1}$ the amount of lost sales, which creates an opportunity cost of $w_{N+1} q_{N+1}$. Define also, the execution cost, given a demand realization $d$,

$$
\begin{aligned}
C(x, d)=\sum_{i=1}^{N+1} v_{i} x_{i}+\quad \min \sum_{i=1}^{N+1} w_{i} q_{i} \\
\text { subject to }\left\{\begin{array}{l}
0 \leq q_{i} \leq x_{i} i=1, \ldots, N, \\
0 \leq q_{N+1}, \\
\sum_{i=1}^{N+1} q_{i}=d
\end{array}\right.
\end{aligned}
$$

Observe that the execution policy of the buyer (after demand is realized) is to use first the option 
contracts with lower execution costs. Hence, the buyer's profit is $\Pi(x, D)=p D-C(x, D)$. Thus, the expected profit is $\bar{\Pi}(x)=p E[D]-E[C(x, D)]$.

Let $y_{0}=0$ and

$$
y_{i}=x_{1}+\ldots+x_{i} \text { for } i=1, \ldots, N .
$$

Then, $V(y)=\bar{\Pi}(x)$ satisfies for $i=1, \ldots, N$, see [13],

$$
\frac{d V}{d y_{i}}(y)=\left(v_{i+1}-v_{i}\right)+\left(w_{i+1}-w_{i}\right) \operatorname{Pr}\left[D \geq y_{i}\right] .
$$

Equation (2) thus provides the structure of the buyer's optimal portfolio which is determined by the c.d.f. of customer demand. One can observe that the marginal value of increasing $y_{i}$ while keeping the rest fixed (i.e., increasing $x_{i}$ and decreasing $x_{i+1}$, in fact replacing capacity installed at $i$ by capacity at $i+1)$ is equal to the increase in reservation cost, $v_{i+1}-v_{i}$ per unit, plus the increase in average execution cost, $\left(w_{i+1}-w_{i}\right) \operatorname{Pr}\left[D \geq y_{i}\right]$ per unit. Under Assumption 2, when there are no identical bids from the suppliers, the profit is a strictly concave function of $\left(y_{1}, \ldots, y_{N}\right)$ defined over the set

$$
P=\left\{\left(y_{1}, \ldots, y_{N}\right) \in \mathbb{R}^{N} \mid 0 \leq y_{1} \leq \ldots \leq y_{N}\right\}
$$

Strict concavity implies that the optimal solution is unique. Thus, in the game analyzed in this paper, the leaders know exactly how the follower behaves.

To characterize the optimal portfolio, $\left(x_{1}^{*}, \ldots, x_{N}^{*}\right)$, we need the following definitions.

Definition 1 Supplier $i$ is called active if $x_{i}^{*}>0$. Otherwise, it is called inactive.

Definition 2 Given a set of $t$ different pairs $\left\{\left(a_{1}, b_{1}\right), \ldots,\left(a_{t}, b_{t}\right)\right\}$ with $a_{1} \leq \ldots \leq a_{t}$, the winning set is the minimal subset $S=\left\{i_{1}, \ldots, i_{k}\right\}$ of these points such that:

(a) $a_{i_{1}} \leq \ldots \leq a_{i_{k}}$

(b) for $1 \leq i<i_{1}, b_{i}-b_{i_{1}} \geq-\left(a_{i}-a_{i_{1}}\right)$;

(c) for $j=2, \ldots, k$, for $i_{j-1}<i<i_{j}, b_{i}-b_{i_{j}} \geq-\left(\frac{b_{i_{j-1}}-b_{i_{j}}}{a_{i_{j}}-a_{i_{j-1}}}\right)\left(a_{i}-a_{i_{j}}\right)$;

(d) for $i_{k} \leq i \leq t, b_{i} \geq b_{i_{k}}$.

$i_{1}, \ldots, i_{k}$ are called winning points among the $t$ pairs. Also, the lower envelope is the curve $Z^{(\mathbf{a}, \mathbf{b})}(\cdot)$ defined as follows

$$
Z^{(\mathbf{a}, \mathbf{b})}(u)= \begin{cases}b_{i_{1}}-\left(u-a_{i_{1}}\right) & \text { for } u \leq a_{i_{1}} \\ b_{i_{2}}-\left(\frac{b_{i_{1}}-b_{i_{2}}}{a_{i_{2}}-a_{i_{1}}}\right)\left(u-a_{i_{2}}\right) & \text { for } a_{i_{1}} \leq u \leq a_{i_{2}} \\ \vdots & \\ b_{i_{k}}-\left(\frac{b_{i_{k-1}}-b_{i_{k}}}{a_{i_{k}}-a_{i_{k-1}}}\right)\left(u-a_{i_{k}}\right) & \text { for } a_{i_{k-1}} \leq u \leq a_{i_{k}} \\ b_{i_{k}} & \text { for } a_{i_{k}} \geq u,\end{cases}
$$


These definitions, together with Equation (2), are used to characterize the optimal portfolio explicitly, as is done in the next proposition.

Proposition 1 Supplier $i, i=1, \ldots, N$, is active if and only if $i$ is a winning point of $\left\{\left(w_{1}, v_{1}\right)\right.$, $\left.\ldots,\left(w_{N+1}, v_{N+1}\right)\right\}$.

All the proofs are presented in the appendix.

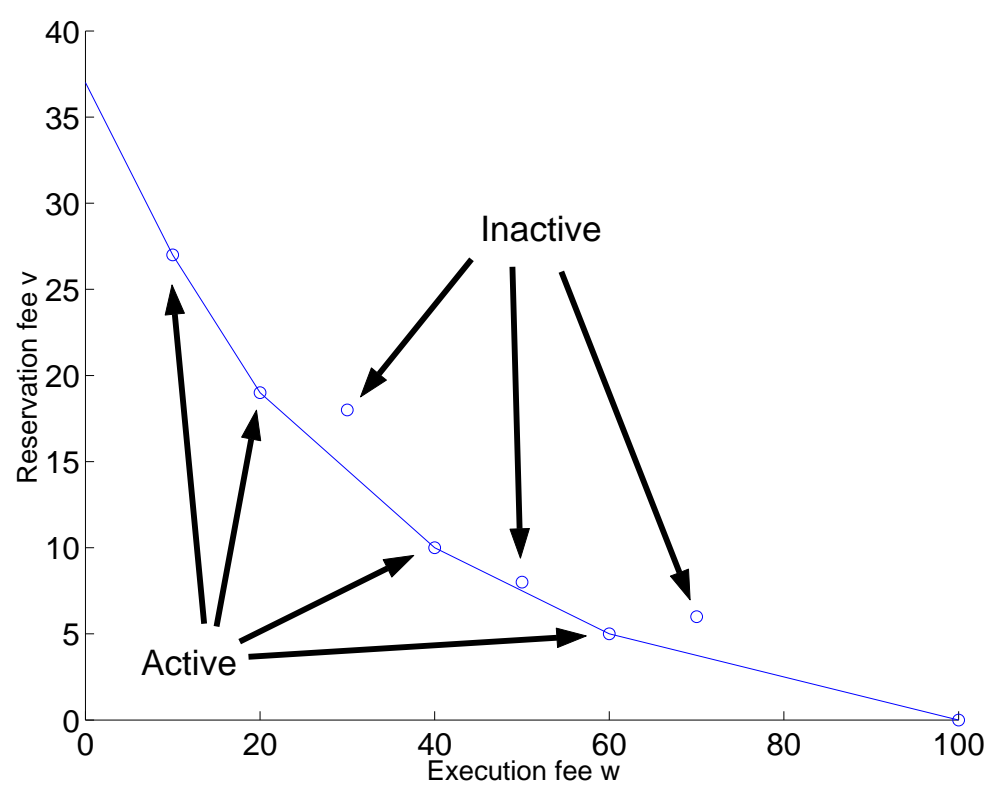

Figure 1: Illustration of active and inactive bids.

The winning points, i.e., all the active suppliers, can be determined graphically, see Figure 1. Plot the pairs $\left(w_{i}, v_{i}\right)$ in a graph with the $w_{i}$ in the $\mathrm{x}$ coordinate and the $v_{i}$ in the y coordinate. Add also the point $(p, 0)$. Determine the convex hull of the points, and in particular find the extreme points on the lower envelope as defined in Definition 2 ; these are the points $i_{1}<\ldots<i_{k}$.

Hence, the lower envelope is piecewise linear and convex. The segments have increasing slopes or equivalently decreasing negative slopes, that is,

$$
1>\frac{v_{i_{1}}-v_{i_{2}}}{w_{i_{2}}-w_{i_{1}}}>\ldots>\frac{v_{i_{k-1}}-v_{i_{k}}}{w_{i_{k}}-w_{i_{k-1}}}>0
$$

The buyer's optimal strategy is to include only suppliers on the lower envelope that form segments with negative slopes between 0 and 1 . This implies that $v_{i_{1}}+w_{i_{1}}>\ldots>v_{i_{k}}+w_{i_{k}}$ and $v_{i_{1}}>\ldots>v_{i_{k}}$. With these definitions, and recalling $y_{0}=0$, the optimal portfolio is defined by

$$
y_{i}^{*}=\left\{\begin{array}{l}
\bar{F}^{-1}\left(\frac{v_{i_{j}}-v_{i_{j+1}}}{w_{i_{j+1}}-w_{i_{j}}}\right) \text { if } i=i_{j}, j=1, \ldots, k-1, \\
y_{i-1}^{*} \text { for all others. }
\end{array}\right.
$$


The vector $\mathbf{x}^{*}$ follows directly from $\mathbf{y}^{*}$. In particular $x_{i}^{*}=0$ for $i$ different than $i_{1}, \ldots, i_{k}$.

Recalling Equation (2), this portfolio structure yields that the buyer reserves capacity with low execution cost $w$ and high reservation cost $v$ to cover the demand with higher realization probability; and uses capacity with high execution cost and low reservation cost to cover the right-tail of the demand.

\section{The Suppliers' Behavior}

Given that the buyer uses a portfolio approach as described in the previous section, each supplier will set its reservation and execution price to maximize its expected profit, taking into account the behavior of other suppliers.

Consider the decision of supplier $i, i=1, \ldots, N$. It is confronted by bids from other suppliers. Let $\left(\mathbf{w}_{-i}, \mathbf{v}_{-i}\right)$ be the vector representing all other bids with the additional point $\left(w_{N+1}=p, v_{N+1}=0\right)$. We assume these bids to be fixed in this section, and characterize the best bidding strategy of the supplier in response to them.

Given the bids in the vector $\left(\mathbf{w}_{-i}, \mathbf{v}_{-i}\right)$, we can identify the buyer's optimal procurement strategy. Assume that, within these bids, there are $k \leq N$ active suppliers, indexed from 1 to $k$, with $w_{i_{1}} \leq \ldots \leq w_{i_{k}}$ (one of the suppliers might be the dummy supplier with parameters $(p, 0)$ ). The buyer's best procurement strategy, excluding the bid of $i$ for the moment, is to set

$$
\left\{\begin{array}{l}
y_{i_{j}}^{-i}=\bar{F}^{-1}\left(\frac{v_{i_{j}}-v_{i_{j+1}}}{w_{i_{j+1}}-w_{i_{j}}}\right) j=1, \ldots, k-1, \\
y_{i_{k}}^{-i}=\bar{F}^{-1}(0) .
\end{array}\right.
$$

If supplier $i$ places a bid $\left(w_{i}, v_{i}\right)$, the buyer's optimal solution may change to take this bid into account. Of course, suppliers that were not active before are not going to be active with the new bid from supplier $i$. However, it is entirely possible that some suppliers may become inactive when supplier $i$ enters with the bid $\left(w_{i}, v_{i}\right)$. Finally, supplier $i$ may capture zero capacity if its bid makes it inactive. Clearly, in this case, if supplier $i$ is inactive, we can withdraw it from the pool of bids and consequently the capacities allocated to the other suppliers remain unchanged. This happens when $\left(w_{i}, v_{i}\right)$ is above the lower envelope which is described by the function $Z^{\left(\mathbf{w}_{-i}, \mathbf{v}_{-i}\right)}(\cdot)$ in Definition 2. Thus, when $v_{i} \geq Z^{\left(\mathbf{w}_{-i}, \mathbf{v}_{-i}\right)}\left(w_{i}\right)$, supplier $i$ is inactive and its profit is $\Pi=0$. We define this bidding region which makes $i$ inactive as

$$
A_{\text {OUT }}=\left\{(w, v) \in \mathbb{R}_{+}^{2} \mid v \geq Z^{\left(\mathbf{w}_{-i}, \mathbf{v}_{-i}\right)}(w)\right\} .
$$

If supplier $i$ 's bid is not in that region, then supplier $i$ becomes active. Adding bid $\left(w_{i}, v_{i}\right)$ to the rest of the bids may change the convex hull of the points in two different ways: 
- Supplier $i$ becomes the first active supplier, i.e., there exist $h \in\{1, \ldots, k\}$ such that suppliers $i, i_{h}, \ldots, i_{k-1}$ are active and suppliers $i_{1}, \ldots, i_{h-1}$ are inactive. We define this region as $A_{0 h}$.

$$
A_{0 h}=\left\{\begin{array}{l|l}
(w, v) \in \mathbb{R}_{+}^{2} & \begin{array}{l}
v-v_{i_{1}} \leq-\left(w-w_{i_{1}}\right) \\
v-v_{i_{h}} \leq-\left(\frac{v_{i_{h-1}}-v_{i_{h}}}{w_{i_{h}}-w_{i_{h-1}}}\right)\left(w-w_{i_{h}}\right) \quad(\text { only if } h>1) \\
v-v_{i_{h}} \geq-\left(\frac{v_{i_{h}}-v_{i_{h+1}}}{w_{i_{h+1}}-w_{i_{h}}}\right)\left(w-w_{i_{h}}\right)
\end{array}
\end{array}\right\}
$$

- Supplier $i$ is not the first active supplier, i.e., there exist $l \in\{1, \ldots, k-1\}$ and $h \in\{1, \ldots, k\}$, $h>l$, such that suppliers $i, i_{1}, \ldots, i_{l}, i_{h}, \ldots, i_{k}$ are active and $i_{l+1}, \ldots, i_{h-1}$ inactive. We define this region as $A_{l h}$.

$$
A_{l h}=\left\{\begin{array}{l|l}
(w, v) \in \mathbb{R}_{+}^{2} & \begin{array}{l}
v-v_{i_{l}} \geq-\left(\frac{v_{i_{l-1}}-v_{i_{l}}}{w_{i_{l}}-w_{i_{l-1}}}\right)\left(w-w_{i_{l}}\right) \\
\text { or } \left.v-v_{i_{1}} \geq-\left(w-w_{i_{1}}\right) \text { if } l=1\right) \\
v-v_{i_{l}} \leq-\left(\frac{v_{i_{l}}-v_{i_{l+1}}}{w_{i_{l+1}}-w_{i_{l}}}\right)\left(w-w_{i_{l}}\right) \\
v-v_{i_{h}} \leq-\left(\frac{v_{i_{h-1}}-v_{i_{h}}}{w_{i_{h}}-w_{i_{h-1}}}\right)\left(w-w_{i_{h}}\right) \\
v-v_{i_{h}} \geq-\left(\frac{v_{i_{h}}-v_{i_{h+1}}}{w_{i_{h+1}}-w_{i_{h}}}\right)\left(w-w_{i_{h}}\right)
\end{array}
\end{array}\right\}
$$

These regions are illustrated in Figure 2. Intuitively, a bid in region $A_{l h}$ implies that supplier $i$ forces suppliers $i_{l+1}, \ldots, i_{h-1}$ out of the market, i.e., these suppliers receive zero capacity allocation.

\subsection{The supplier's profit}

The capacity allocated by the buyer to supplier $i, x_{i}$, if $i$ bids in $A_{l h}, l>0$, is $x_{i}=y_{i+}-y_{i-}$ where $y_{i+}$ and $y_{i-}$ are given by the following set of equations ${ }^{5}$. We drop the sub-index $i$ to simplify notation, and use $l, h$ instead of $i_{l}, i_{h}$.

$$
\bar{F}\left(y_{-}\right)=\frac{v_{l}-v}{w-w_{l}} \text { and } \bar{F}\left(y_{+}\right)=\frac{v-v_{h}}{w_{h}-w} .
$$

The case of $l=0$ is special, since $y_{-}=0$ by construction. Hence, in that case,

$$
y_{-}=0 \text { and } \bar{F}\left(y_{+}\right)=\frac{v-v_{h}}{w_{h}-w} .
$$

The expected profit of supplier $i$ in this case is thus

$$
\Pi=(v-f)\left(y_{+}-y_{-}\right)+(w-c) \mathbb{E}\left[\min \left\{\max \left(D-y_{-}, 0\right), y_{+}-y_{-}\right\}\right],
$$

where the first part is the profit made on reserving capacity, and the second part the expected execution profit (execution occurs for any demand $D$ higher than $y_{-}$, up to the reserved capacity).

\footnotetext{
${ }^{5}$ Since $(w, v) \in A_{l h}$, one must keep in mind that $\left(y_{-}, y_{+}\right)$is constrained. Specifically, $y_{i_{l-1}}^{-i} \leq y_{-} \leq y_{i_{l}}^{-i}$ (for $\left.l>0\right), y_{i_{h-1}}^{-i} \leq y_{+} \leq$ $y_{i_{h}}^{-i}$ and therefore $y_{-} \leq y_{+}$(moreover, if $y_{-}=y_{+}$, supplier $i$ is in $A_{O U T}$ and is thus inactive).
} 


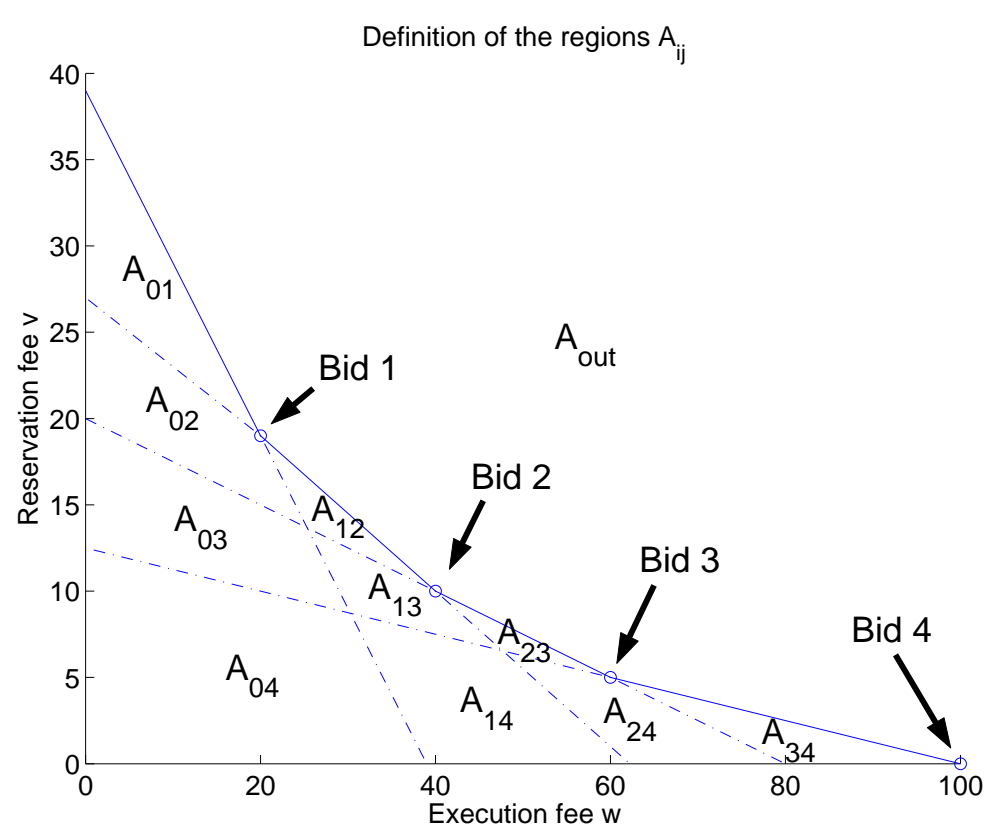

Figure 2: Division of the bidding strategies in different regions. Intuitively, a bid in region $A_{l h}$ implies that supplier $i$ forces suppliers $i_{l+1}, \ldots, i_{h-1}$ out of the market, i.e., these suppliers receive zero capacity allocation.

Since $E\left[\min \left\{\max \left(D-y_{-}, 0\right), y_{+}-y_{-}\right\}\right]=\int_{y_{-}}^{y_{+}}\left(u-y_{-}\right) f(u) d u+\left(y_{+}-y_{-}\right) \bar{F}\left(y_{+}\right)$, integration in parts yields

$$
\Pi=(v-f)\left(y_{+}-y_{-}\right)+(w-c) \int_{y_{-}}^{y_{+}} \bar{F}(u) d u .
$$

Using Equation (8), one can express $(w, v)$ as a function of $y_{-}$and $y_{+}$when $y_{-}<y_{+}$, since $f(\cdot)>0$. Specifically,

$$
\begin{aligned}
& v=v_{h}+\bar{F}\left(y_{+}\right) \frac{-\left(v_{l}-v_{h}\right)+\bar{F}\left(y_{-}\right)\left(w_{h}-w_{l}\right)}{\bar{F}\left(y_{-}\right)-\bar{F}\left(y_{+}\right)}=v_{l}-\bar{F}\left(y_{-}\right) \frac{\left(v_{l}-v_{h}\right)-\bar{F}\left(y_{+}\right)\left(w_{h}-w_{l}\right)}{\bar{F}\left(y_{-}\right)-\bar{F}\left(y_{+}\right)} \\
& w=w_{h}-\frac{-\left(v_{l}-v_{h}\right)+\bar{F}\left(y_{-}\right)\left(w_{h}-w_{l}\right)}{\bar{F}\left(y_{-}\right)-\bar{F}\left(y_{+}\right)}=w_{l}+\frac{\left(v_{l}-v_{h}\right)-\bar{F}\left(y_{+}\right)\left(w_{h}-w_{l}\right)}{\bar{F}\left(y_{-}\right)-\bar{F}\left(y_{+}\right)} .
\end{aligned}
$$

This implies that we can express $\Pi$ using $y_{-}$and $y_{+}$instead of $v$ and $w$. Within $A_{l h}, l>0$,

$$
\begin{aligned}
\Pi(w, v)=J_{l h}\left(y_{-}, y_{+}\right)=\left\{\begin{array}{l}
\left(v_{h}-f\right)\left(y_{+}-y_{-}\right)+\left(w_{h}-c\right) \int_{y_{-}}^{y_{+}} \bar{F}(u) d u \\
-\left[\frac{-\left(v_{l}-v_{h}\right)+\bar{F}\left(y_{-}\right)\left(w_{h}-w_{l}\right)}{\bar{F}\left(y_{-}\right)-\bar{F}\left(y_{+}\right)} \int_{y_{-}}^{y_{+}}\left(\bar{F}(u)-\bar{F}\left(y_{+}\right)\right) d u\right.
\end{array}\right. \\
=\left\{\begin{array}{l}
\left(v_{l}-f\right)\left(y_{+}-y_{-}\right)+\left(w_{l}-c\right) \int_{y_{-}} \bar{F}(u) d u \\
-\left[\frac{\left(v_{l}-v_{h}\right)-\bar{F}\left(y_{+}\right)\left(w_{h}-w_{l}\right)}{\bar{F}\left(y_{-}\right)-\bar{F}\left(y_{+}\right)} \int_{y_{-}}^{y_{+}}\left(\bar{F}\left(y_{-}\right)-\bar{F}(u)\right) d u\right.
\end{array}\right.
\end{aligned}
$$


When $l=0$, the transformation described in Equation (9) is not well defined, since different values of $(w, v)$ yield the corresponding $\left(y_{-}=0, y_{+}\right)$. We observe that for a given $y_{+}, y_{-}=0$, the profit with a bid $w=w_{h}-t$ and $v=v_{h}+\bar{F}\left(y_{+}\right) t, t \geq 0$, is,

$$
\begin{aligned}
\Pi & =\left(v_{h}+\bar{F}\left(y_{+}\right) t-f\right) y_{+}+\left(w_{h}-t-c\right) \int_{0}^{y_{+}} \bar{F}(u) d u \\
& =\left(v_{h}-f\right) y_{+}+\left(w_{h}-c\right) \int_{0}^{y_{+}} \bar{F}(u) d u-t \int_{0}^{y_{+}}\left(\bar{F}(u)-\bar{F}\left(y_{+}\right)\right) d u
\end{aligned}
$$

Thus, to maximize $\Pi$ it is best for the supplier to select $t$ as small as possible, within $A_{0 h}$ : we set

$$
\begin{gathered}
v+w=v_{i_{1}}+w_{i_{1}} \\
\frac{v-v_{h}}{w_{h}-w}=\bar{F}\left(y_{+}\right) .
\end{gathered}
$$

This justifies the extension of Equation (8) for $l=0$. Consequently,

$$
\Pi(w, v)=J_{0 h}\left(y_{+}\right)=\left\{\begin{array}{l}
\left(v_{h}-f\right) y_{+}+\left(w_{h}-c\right) \int_{0}^{y_{+}} \bar{F}(u) d u \\
-\left[\frac{\left(v_{h}+w_{h}\right)-\left(v_{i_{1}}+w_{i_{1}}\right)}{1-\bar{F}\left(y_{+}\right)}\right] \int_{0}^{y_{+}}\left(\bar{F}(u)-\bar{F}\left(y_{+}\right)\right) d u
\end{array}\right.
$$

Finally, the problem faced by supplier $i$ is:

$$
\sup _{(w, v)} \Pi(w, v)=\max \left(0, \max _{l=0, \ldots, k-1, h=l+1, \ldots, k} \sup _{\left(y_{-}, y_{+}\right)} J_{l h}\left(y_{-}, y_{+}\right)\right)
$$

The optimization problem is defined as a supremum of profit, in terms of either $(w, v)$ or $\left(y_{-}, y_{+}\right)$. As we shall see later, when optimizing on $(w, v)$, there does not always exist an optimal solution, and the supremum may be obtained by bidding arbitrarily close to another supplier. However, when using $\left(y_{-}, y_{+}\right)$as decision variables, an optimal solution is always obtained.

Since $\bar{F}(\cdot)$ is differentiable over $(\underline{d}, \bar{d})$, the expected profit is differentiable in $\left(y_{-}, y_{+}\right)$:

$$
\begin{aligned}
& \frac{d J_{l h}}{d y_{-}}=\left(f-v_{l}\right)+\left(c-w_{l}\right) \bar{F}\left(y_{-}\right)+f\left(y_{-}\right)\left[\frac{\left(v_{l}-v_{h}\right)-\bar{F}\left(y_{+}\right)\left(w_{h}-w_{l}\right)}{\left(\bar{F}\left(y_{-}\right)-\bar{F}\left(y_{+}\right)\right)^{2}}\right] \int_{y_{-}}^{y_{+}}\left(\bar{F}(u)-\bar{F}\left(y_{+}\right)\right) d u \\
& \frac{d J_{l h}}{d y_{+}}=\left(v_{h}-f\right)+\left(w_{h}-c\right) \bar{F}\left(y_{+}\right)-f\left(y_{+}\right)\left[\frac{-\left(v_{l}-v_{h}\right)+\bar{F}\left(y_{-}\right)\left(w_{h}-w_{l}\right)}{\left(\bar{F}\left(y_{-}\right)-\bar{F}\left(y_{+}\right)\right)^{2}}\right] \int_{y_{-}}^{y_{+}}\left(\bar{F}\left(y_{-}\right)-\bar{F}(u)\right) d u
\end{aligned}
$$

Notice that $\left(v_{l}-v_{h}\right)-\bar{F}\left(y_{+}\right)\left(w_{h}-w_{l}\right) \geq 0$ and $-\left(v_{l}-v_{h}\right)+\bar{F}\left(y_{-}\right)\left(w_{h}-w_{l}\right) \geq 0$ hold; we shall use this observation later.

\subsection{Border distributions}

Maximizing $J_{l h}$ on $\left(y_{-}, y_{+}\right)$, such that $(w, v) \in A_{l h}$, may yield, in general, interior solutions or extreme solutions. It turns out that they are always extreme solutions for a class of customer demand distributions. A customer demand distribution is called border demand distribution when it satisfies the following property. 
Property 1 (border distribution) For any supplier, for any region $A_{l h}$, defined by Equations (6) or (7), there is an optimal bid $(w, v)$ that belongs to the border of the region.

The property implies that for any supplier bidding in region $A_{l h}$, there is an optimal bid on the boundary of region $A_{l h}$, for any cost parameters. For instance, for a supplier bidding in region $A_{12}$ of Figure 2, there is an optimal bid on the boundary of $A_{12}$ with either $A_{02}$ or $A_{13}$ or $A_{\text {OUT }}$.

Interestingly, a wide class of demand distributions, the class of log-concave distributions, satisfies the border distribution property. For any distribution in this class, the logarithm of the demand's p.d.f. is concave. It is easy to verify that the class of log-concave includes distributions such as uniform, exponential, normal, etc.

Theorem 1 A log-concave demand distribution, i.e., with $\log (f)$ concave, is a border distribution.

The proof of this result is presented in the appendix and can also be found in Martínez-de-Albéniz [12].

Under Property 1, the suppliers will place their bids in the border of some region. The property allows us to determine their optimal bids.

\subsection{Optimal bids}

As we will soon see, it is of particular interest to examine the situation in $A_{l h}$ where there is no active supplier between $l$ and $h$ and both $l$ and $h$ are active. Notice that these are all the regions that share an edge with $A_{O U T}$. When we know that the optimal bid is in this region, we can characterize the optimal bid.

Consider supplier $i$ bidding in such a region, $A_{l h}$, and define $y_{m}$ to be the cumulative quantity captured by suppliers $i_{1}, \ldots, i_{l}$ when $i$ is absent, i.e.,

$$
\bar{F}\left(y_{m}\right)=\frac{v_{l}-v_{h}}{w_{h}-w_{l}} .
$$

The constraint of being in $A_{l h}$ can be written as $y_{0}:=y_{i_{l-1}}^{-i} \leq y_{-} \leq y_{m} \leq y_{+} \leq y_{3}:=y_{i_{h}}^{-i}$ where $y_{i_{l-1}}^{-i}$ and $y_{i_{h}}^{-i}$ are defined in Equation (5).

If the bid of supplier $i$ does not make $l$ or $h$ inactive, we can derive useful properties. In this case, the optimal bid cannot be such that $y_{-}=y_{0}$ (because it makes $i_{l}$ inactive) or $y_{+}=y_{3}\left(i_{h}\right.$ inactive). Therefore, since it is optimal to bid on the border of the region, it must be that $y_{-}=y_{m}$ or $y_{+}=y_{m}$ is optimal. These imply that supplier $i$ bids the same $(w, v)$ as $l$ or $h$.

In the first case, i.e., when $y_{-}=y_{m}$ is optimal, recall that $-\left(v_{l}-v_{h}\right)+\bar{F}\left(y_{-}\right)\left(w_{h}-w_{l}\right)=0$ so from Equation (12)

$$
\frac{d J_{l h}}{d y_{+}}=\left(v_{h}-f\right)+\left(w_{h}-c\right) \bar{F}\left(y_{+}\right)
$$

and therefore we must have that $c \leq w_{h}$ and

$$
\bar{F}\left(y_{+}\right)=\frac{f-v_{h}}{w_{h}-c} .
$$


Similarly, when $y_{+}=y_{m}$ is optimal,

$$
\frac{d J_{l h}}{d y_{-}}=\left(f-v_{l}\right)+\left(c-w_{l}\right) \bar{F}\left(y_{-}\right)
$$

hence $w_{l} \leq c$ and

$$
\bar{F}\left(y_{-}\right)=\frac{v_{l}-f}{c-w_{l}} .
$$

We summarize these results in the next theorem.

Theorem 2 Given a border distribution, assume that, for a supplier with costs $(c, f)$, the optimal bid belongs to some unique region $A_{l h}, l>0$, where there is no active supplier between $l$ and $h$. Define $y_{m}$ as in Equation (13) and hence having $(w, v) \in A_{l h}$ is equivalent, for some $y_{0}, y_{3}$, to $y_{0} \leq y_{-} \leq y_{m} \leq y_{+} \leq y_{3}$. Define $y_{1}$ and $y_{2}$ as follows,

$$
\begin{aligned}
& \bar{F}\left(y_{1}\right)=\frac{v_{l}-f}{c-w_{l}}, \\
& \bar{F}\left(y_{2}\right)=\frac{f-v_{h}}{w_{h}-c} .
\end{aligned}
$$

Then, one and only one case from the following is true.

- either $y_{0} \leq y_{1} \leq y_{m}$ and $y_{2}>y_{3}$, and $\left(w^{*}, v^{*}\right)=\left(w_{l}, v_{l}\right), y_{+}^{*}=y_{m}$ and $y_{-}^{*}=y_{1}$,

- or $y_{0}>y_{1}$ and $y_{m} \leq y_{2} \leq y_{3}$, and $\left(w^{*}, v^{*}\right)=\left(w_{h}, v_{h}\right), y_{-}^{*}=y_{m}$ and $y_{+}^{*}=y_{2}$,

- or $y_{0} \leq y_{1} \leq y_{m} \leq y_{2} \leq y_{3} ;\left(w^{*}, v^{*}\right)=\left(w_{l}, v_{l}\right), y_{+}^{*}=y_{m}$ and $y_{-}^{*}=y_{1}$, only if

$$
\frac{\int_{y_{1}}^{y_{m}}\left[\bar{F}\left(y_{1}\right)-\bar{F}(u)\right] d u}{\bar{F}\left(y_{1}\right)-\bar{F}\left(y_{m}\right)} \geq \frac{\int_{y_{m}}^{y_{2}}\left[\bar{F}(u)-\bar{F}\left(y_{2}\right)\right] d u}{\bar{F}\left(y_{m}\right)-\bar{F}\left(y_{2}\right)} ;
$$

$\left(w^{*}, v^{*}\right)=\left(w_{h}, v_{h}\right), y_{-}^{*}=y_{m}$ and $y_{+}^{*}=y_{2}$, only if

$$
\frac{\int_{y_{1}}^{y_{m}}\left[\bar{F}\left(y_{1}\right)-\bar{F}(u)\right] d u}{\bar{F}\left(y_{1}\right)-\bar{F}\left(y_{m}\right)} \leq \frac{\int_{y_{m}}^{y_{2}}\left[\bar{F}(u)-\bar{F}\left(y_{2}\right)\right] d u}{\bar{F}\left(y_{m}\right)-\bar{F}\left(y_{2}\right)} .
$$

Intuitively, the theorem shows that there are two candidate optimal bids when we know that the optimal bid is in a given region $A_{l h}$, and there are no active suppliers in between $i_{l}$ and $i_{h}$. First, the supplier may choose to set $\left(w^{*}, v^{*}\right)=\left(w_{l}, v_{l}\right)$, together with $y_{-}^{*}=y_{1}$. Since the supplier profit function is not well-defined when two suppliers submit identical bids, this means that the best strategy of the supplier is to place a bid arbitrarily close to $\left(w_{l}, v_{l}\right)$ while ensuring that $y_{-}=$ $y_{1}$. Thus, there is no optimal bid when defined through $(w, v)$, but there is an optimal bid when defined with $\left(y_{-}, y_{+}\right)$. In practice, this means that a sequence of bids such that $w^{\delta}=w_{l}+\delta$ and $v^{\delta}=v_{l}-\bar{F}\left(y_{1}\right) \delta$ approaches the highest expected profit, when $\delta \rightarrow 0$ and positive. Alternatively, the supplier may choose to set $\left(w^{*}, v^{*}\right)=\left(w_{h}, v_{h}\right)$, together with $y_{+}^{*}=y_{2}$. The profit function 
is not well defined in this case as well. Similarly, a sequence of bids such that $w^{\delta}=w_{h}-\delta$ and $v^{\delta}=v_{h}+\bar{F}\left(y_{1}\right) \delta$ approaches the highest expected profit, when $\delta \rightarrow 0$ and positive.

Interestingly, no other situations are possible at optimality, since the demand distribution satisfies the border property and there is no optimal solution outside $A_{l h}$ (thus discarding a strategy where $y_{-}=y_{0}$ or $\left.y_{+}=y_{3}\right)$.
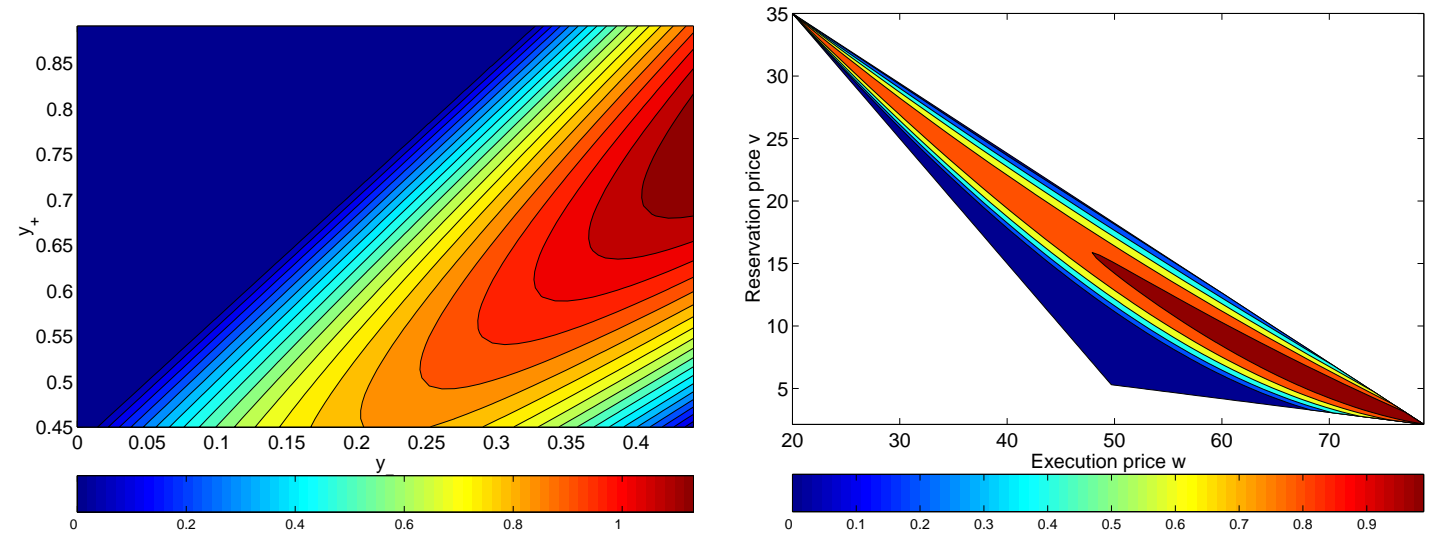

Figure 3: Expected supplier profit (only non-negative values are shown, for better readability), as a function of $\left(y_{-}, y_{+}\right)$(left figure), and as a function of $(w, v)$ (right figure). We use here a [0,1]-uniform demand, with competing bids $(0,55),(20,35),(80,2)$ and $(100,0)$. The supplier costs are $c=55$ and $f=8$. We calculate the expected profit obtained by bidding in $A_{23}$, i.e., between the second and third bids, $(20,35)$ and $(80,2)$.

Figure 3 illustrates the optimal bid discussed in the theorem. In the figure we show iso-profit curves as a function of both $\left(y_{-}, y_{+}\right)$, on the left, and of $(w, v)$, on the right, for a [0,1]-uniform demand, with competing bids $(0,55),(20,35),(80,2)$ and $(100,0)$. The supplier costs are $c=55$ and $f=8$. We calculate the expected profit obtained by bidding in $A_{23}$, i.e., between the second and third bids, $(20,35)$ and $(80,2)$, which implies $y_{0}=0, y_{m}=0.5$ and $y_{3}=0.9$. As one can see, the figure on the left shows that it is optimal to set $y_{-}^{*}=y_{m}=0.5$ and $y_{+}^{*}=y_{2}=0.76$, which yields higher profit than the other candidate bid $y_{-}=y_{1}=0.2286$ and $y_{+}=y_{m}=0.5$. This corresponds to placing a bid very close to $(w, v)=(80,2)$, as seen in the right figure. Note that the profit function is discontinuous at $(w, v)=(80,2)$, which implies that the optimal bid should be $w^{\delta}=80-\delta, v^{\delta}=2+\bar{F}(0.76) \delta=2+0.34 \delta$, for small $\delta>0$.

\section{Game Equilibria}

We analyze the game in which the suppliers compete for selling capacity. This section studies the equilibria of this game in pure strategies.

Consider first the following example. There are $N=2$ suppliers with costs $\left(c_{1}, f_{1}\right)=(0,60)$ and $\left(c_{2}, f_{2}\right)=(75,5)$. The demand is uniformly distributed in $[0,1]$. The selling price is $p=100$, and 
hence the dummy supplier posts a bid $(p, 0)$. Consider the situation when both suppliers submit two bids that are very close to $(60,12)$. Are these bids in equilibrium? In other words, does each supplier maximize its profit given the competitor's bid? As we demonstrate below, this is not the case.

Indeed, Figure 4 shows the profit functions of each supplier as a function of their bid $(w, v)$. As one can see, both are maximized by setting $(w, v)=(60,12)$. Since the profit maximizer is not well defined in $(w, v)$, as seen in the previous section, supplier 1's profit function is maximized by setting $y_{1-}^{*}=0$ and $y_{1+}^{*}=0.2$, and supplier 2's profit function is maximized by $y_{2-}^{*}=0.5333$ and $y_{2+}^{*}=0.8$. Hence, this cannot be an equilibrium, since the suppliers do not agree on a capacity allocation. However, if the suppliers' bids would yield $y_{1+}^{*}=y_{2-}^{*}$, both suppliers would have no incentive to modify their bids.
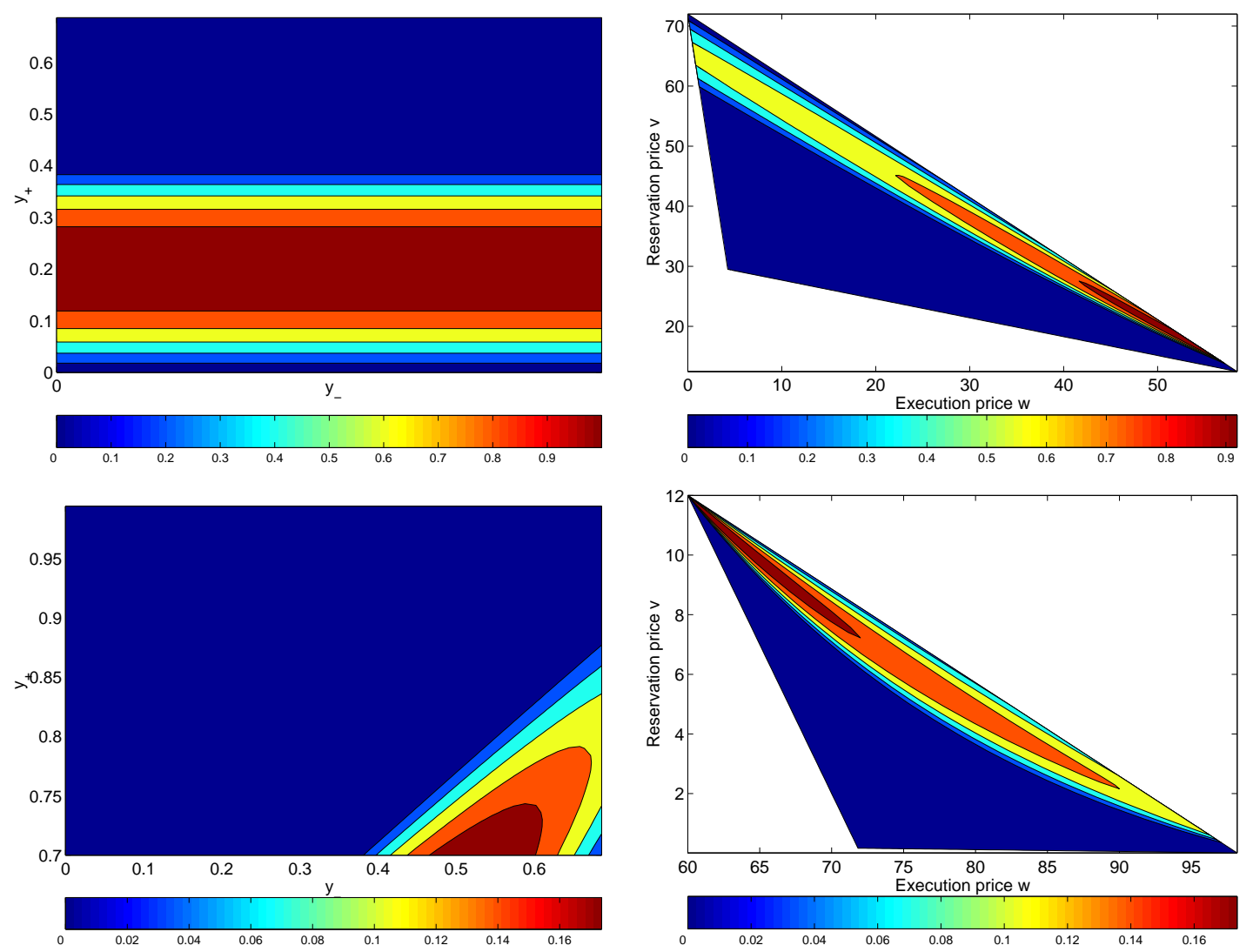

Figure 4: Expected supplier profit (only non-negative values are shown, for better readability), as a function of $\left(y_{-}, y_{+}\right)$(left figures), and as a function of $(w, v)$ (right figures). We use here a [0,1]-uniform demand. The upper figure represents the profit of supplier 1 , with cost $\left(c_{1}, f_{1}\right)=(0,60)$, with competing bids of $(60,12)$ (supplier 2) and (100,0) (dummy supplier). The lower figure represents the profit of supplier 2, with cost $\left(c_{2}, f_{2}\right)=(75,5)$, with competing bids of $(60,12)$ (supplier 1$)$ and $(100,0)$ (dummy supplier).

The example shows that the concept of equilibrium in this game is not well defined when two 
bids are identical. However, using the optimality equations, using $y_{-}$and $y_{+}$, we can determine when a bid situation with ties is stable.

\subsection{Equilibrium conditions}

When the strategies are defined only through $(w, v)$, no equilibria might exist since some supplier's problem may not have an optimal solution, as its profit function is discontinuous when two suppliers submit the same $\operatorname{bid}^{6}$.

To overcome this problem, we consider, instead of Nash equilibrium, the concept of $\epsilon$-equilibrium, as defined in Radner [16] or Fudenberg and Levine [9]. We say a set of pure strategies $\left(w_{i}, v_{i}\right)_{i=1, \ldots, N}$ is an $\epsilon$-equilibrium of the bidding game when, for each supplier,

$$
\Pi_{i}\left(w_{i}, v_{i}, \mathbf{w}_{-i}, \mathbf{v}_{-i}\right) \geq \sup _{(w, v)} \Pi_{i}\left(w, v, \mathbf{w}_{-i}, \mathbf{v}_{-i}\right)-\epsilon .
$$

For $\epsilon \rightarrow 0$, we characterize the limit of $\epsilon$-equilibria. In other words, we describe what sort of equilibria arises when suppliers choose bids that are very close to the optimum.

In what follows, we say that a set of pure strategies $\left(w_{i}, v_{i}\right)_{i=1, \ldots, N}$ is an equilibrium of the bidding game when there exists, for each supplier $i$, for each $\epsilon,\left(w_{i}^{\epsilon}, v_{i}^{\epsilon}\right)$, such that: (1) $\left(w_{i}^{\epsilon}, v_{i}^{\epsilon}\right) \rightarrow\left(w_{i}, v_{i}\right)$ when $\epsilon \rightarrow 0$, and (2) for each $\epsilon$,

$$
\Pi_{i}\left(w_{i}^{\epsilon}, v_{i}^{\epsilon}, \mathbf{w}_{-i}^{\epsilon}, \mathbf{v}_{-i}^{\epsilon}\right) \geq \sup _{(w, v)} \Pi_{i}\left(w, v, \mathbf{w}_{-i}^{\epsilon}, \mathbf{v}_{-i}^{\epsilon}\right)-\epsilon .
$$

Essentially, this definition of equilibrium circumvents the continuity problem of the supplier profit function, and hence makes unnecessary the use of a rationing rule in case of a tie.

In this section, we provide necessary conditions for equilibrium. We do not analyze the existence of pure strategy equilibria, although these can be shown to exist under fairly general assumptions. Usual proof methods may not work because a given supplier's pay-off function is discontinuous, when its bid is equal to some other supplier's bid ${ }^{7}$. Fortunately, we are able to show existence by explicitly constructing an equilibrium, see Martínez-de-Albéniz [12] for the algorithmic details. An example of the algorithm is provided in Section 6.2.

Using the results of the previous section, we can characterize a crucial necessary condition for equilibrium, arising from Proposition 2.

Proposition 2 Consider a border distribution. In any equilibrium, if $\left(w_{i}, v_{i}\right)=\left(w_{j}, v_{j}\right)$ and $\Pi_{i}, \Pi_{j}>0$ (both suppliers are active), then $\left(w_{i}, v_{i}\right)$ belongs in the segment $\left[\left(c_{i}, f_{i}\right) ;\left(c_{j}, f_{j}\right)\right]$.

\footnotetext{
${ }^{6}$ As a consequence, the buyer's problem has multiple optimal solutions and hence it is not clear how demand is allocated to the two suppliers. It can be shown that in general, when the splitting is pre-determined exogenously, e.g., split the capacity and the allocation $50-50 \%$, no equilibrium exists.

${ }^{7}$ To our knowledge, the best result that we can hope for with a more general approach is existence of mixed-strategy equilibria, following Dasgupta and Maskin [6].
} 
This is a direct consequence of Proposition 2. Intuitively, if two suppliers $i$ and $j$, submit the same bid and are at equilibrium, then it must be true that the quantities that they desire, their optimal $y_{i+}^{*}$ and $y_{j-}^{*}$, must coincide. This results on having the equilibrium bid in the cost segment.

\subsection{Equilibria with efficient suppliers only}

We start by defining the concept of efficiency which leads to a natural and desirable property of equilibria.

Definition 3 We say that supplier $i$ is efficient when $\left(c_{i}, f_{i}\right)$ is a winning point in the set $\left\{\left(c_{1}, f_{1}\right)\right.$, $\left.\ldots,\left(c_{N}, f_{N}\right),(p, 0)\right\}$.

Proposition 3 Assume that supplier $i$ is efficient. Then, in every equilibrium, $\Pi_{i}>0$.

This implies that efficiency guarantees any supplier to be active in any equilibrium outcome. That is, the supplier will receive a positive share of capacity and make some strictly positive profit.

Proposition 4 Given a border distribution, assume that all suppliers are efficient. Then, in every equilibrium, for every pair $(i, j)$, if $c_{i}<c_{j}$ then $w_{i} \leq w_{j}$.

Proposition 4 implies that if all suppliers are efficient, supplier $i, i=1, \ldots, N$, bids in region $A_{i-1} i+1$ in every equilibrium. More importantly, this result confirms the intuition on the suppliers' bidding behavior. No supplier will bid an execution fee, $w$, lower than a competitor's execution fee if the competitor's execution cost is smaller. Put differently, the smaller a supplier's execution cost, $c$, the lower this supplier's execution bid, $w$.

Combining Theorem 2 and Propositions, 2, 3 and 4, we can characterize strong necessary conditions on the equilibria.

Theorem 3 For a border distribution, assume that all the suppliers are efficient. Define $c_{N+1}=$ $w_{N+1}=p, f_{N+1}=v_{N+1}=0$. Then, in every equilibrium, supplier $i, i=2, \ldots, N$, places its bid:

- either $\left(w_{i}, v_{i}\right)=\left(w_{i-1}, v_{i-1}\right)$, and then this bid falls in the segment $\left[\left(c_{i-1}, f_{i-1}\right) ;\left(c_{i}, f_{i}\right)\right]$;

- or $\left(w_{i}, v_{i}\right)=\left(w_{i+1}, v_{i+1}\right)$, and then this bid falls in the segment $\left[\left(c_{i}, f_{i}\right) ;\left(c_{i+1}, f_{i+1}\right)\right]$.

When $i=1$, only the second case is possible, i.e., $\left(w_{1}, v_{1}\right)=\left(w_{2}, v_{2}\right)$, and this common bid falls in the segment $\left[\left(c_{1}, f_{1}\right) ;\left(c_{2}, f_{2}\right)\right]$.

The theorem builds on the optimal behavior of each supplier: when all suppliers are efficient, it is optimal for supplier $i$ either to place a bid equal to the bid of $i-1$ or that of $i+1$, from Theorem 2. This implies that supplier bids will be clustered in groups of two or three suppliers. This is true since according to the theorem either two suppliers bid somewhere in the segment connecting their true cost parameters, or one supplier bids its true costs and two other suppliers place a similar bid 
to this one. Thus, in practice, one will observe less bids than the number of suppliers, roughly half of them. We call this cluster competition, since in equilibrium the market is divided into stable clusters. An example of the clustering is provided in Figure 5.

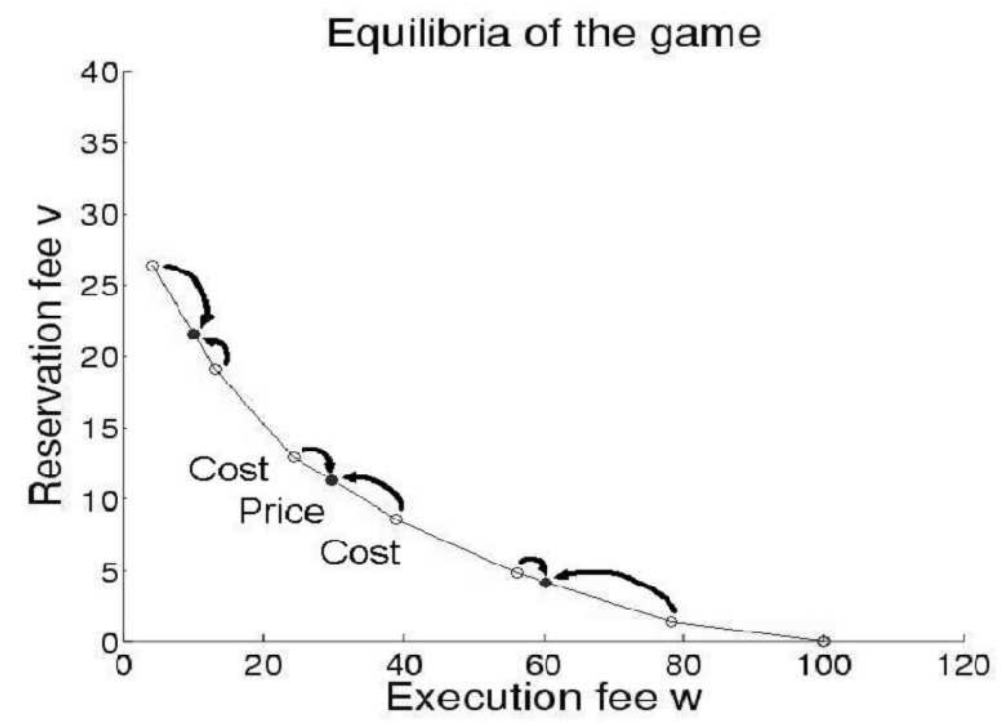

Figure 5: Plot of the costs of six different suppliers, plus dummy supplier at $(c, f)=(100,0)$. Three clusters are formed, with suppliers 1 and 2, 3 and 4, and 5 and 6 placing identical bids.

The type of competition described in this result has some interesting properties. The most striking feature is that more than one supplier will be offering the same bid. One may then wonder whether any supplier in that position should instead reduce its bid a little bit so that it puts its rival out of the market. The answer provided by the theorem is that this is not the case, that is, the additional profit the supplier will receive by reducing its bid is negative. Put differently, all suppliers in the same cluster, i.e., offering the same bid, are better off staying in the cluster rather than trying to outbid the other members of the cluster.

The theorem also suggests that every supplier is competing directly with one of its rival suppliers, i.e., with the supplier who has the next smaller or the next larger execution cost $c$. An important insight from this observation is that, in equilibrium, each supplier's bid will be most sensitive to the bid of its closest competitor, and not to the rest of the bids. This implies that in equilibrium, competition is no longer done on a global basis (among all suppliers) but rather locally (between two or three competing suppliers).

In addition, Theorem 3 can be used to construct equilibria. If an equilibrium exists, supplier $i$ bids the same as $i-1$ or $i+1$. To construct an equilibrium, where supplier $i$ bids the same as supplier $i-1$ (resp. $i+1)$, in the segment $\left[\left(c_{i-1}, f_{i-1}\right) ;\left(c_{i}, f_{i}\right)\right]$ (resp. $\left[\left(c_{i}, f_{i}\right) ;\left(c_{i+1}, f_{i+1}\right)\right]$ ), one must ensure that the supplier realizes a higher profit than bidding the same as $i+1$ (resp. $i-1$ ). 
Consider for example the case where $N=3$, all suppliers are efficient, and $c_{1}<c_{2}<c_{3}<c_{4}=p$. When suppliers 1 and 2 bid their true cost (4, the dummy supplier, also bids its true cost), let $e(3)$ be the supplier whose bid supplier 3 prefers to imitate. That is, $e(3)=2$ if supplier 3 is better off by placing a bid close to the cost of supplier 2 ; and $e(3)=4$ if it is better to place it close to the cost of the dummy supplier, 4 . Then, if $e(3)=2,\left(w_{1}, v_{1}\right)=\left(w_{2}, v_{2}\right)=\left(w_{3}, v_{3}\right)=\left(c_{2}, f_{2}\right)$ is an equilibrium; if $e(3)=4,\left(w_{1}, v_{1}\right)=\left(w_{2}, v_{2}\right)=\left(c_{2}, f_{2}\right),\left(w_{3}, v_{3}\right)=(p, 0)$ is an equilibrium. This approach can be extended to arbitrary $N$, where $e(i)$ is determined for all $i$, and then suppliers are matched so that the proposed bids form an equilibrium. The details can be found in Martínez-de-Albéniz [12].

Finally, observe that the theorem does not rule out the existence of multiple equilibria, and in general the set of equilibria contains multiple possibilities. In any case, this result shows that the possible equilibria belong to the lower envelope of the true suppliers' costs. Such equilibria should satisfy the optimality conditions in Equations (16) and (17). The following example (the one presented at the beginning of this section) illustrates the multiplicity of equilibria.

Example 1 Assume that customer demand is uniformly distributed in $[0,1]$. Let $N=2$ and the true costs be $\left(c_{1}, f_{1}\right)=(0,60),\left(c_{2}, f_{2}\right)=(75,5), p=100$. Both suppliers are efficient. For any $w \in[50,75]$, the following bids form different equilibria:

$$
\left(w_{1}, v_{1}\right)=\left(w_{2}, v_{2}\right)=\left(w, 60-\frac{55}{75} w\right), \quad y_{1}=\frac{20}{75}, \quad y_{2}=\frac{4}{15}+\frac{40}{3(100-w)} .
$$

We should point out that in any of these equilibria, the buyer's expected profit is equal to $\frac{8(150-w)^{2}}{225(100-w)} \geq 64 / 9$. On the other hand, an Expected Vickrey-Clark-Groves (EVCG) auction, which is supply-chain-efficient (see Schummer and Vohra [17] for details), would allocate supplier 1 a profit of $32 / 3-8=8 / 3$, supplier 2 a profit of $32 / 3-8=8 / 3$ and the buyer an expected profit of $32 / 3-8 / 3-8 / 3=16 / 3<64 / 9$. Thus, in this example, the first-price competition environment is preferred by the buyer to the supply-chain-efficient EVCG auction.

The profit functions of suppliers, buyer and the entire supply chain are plotted in Figure 6. As we can see, the profit of supplier 1 is increasing in $w$, the profit of supplier 2 is decreasing in $w$. Thus, among all equilibria, there is no single one that both suppliers prefer: supplier 1 would always prefer an equilibrium with high $w$, while supplier 2 would prefer small $w$. Finally, the buyer's profit is increasing in $w$ (but could be decreasing in other examples), and the total supply chain profit is increasing in $w$.

Finally, to conclude this section, we provide a bound on the inefficiencies created by suppliers' competition. We define the total surplus as follows:

$$
\left.U=(\text { PROFIT OF BUYER })+\sum_{i=1}^{N} \text { (PROFIT OF SUPPLIER } i\right) .
$$




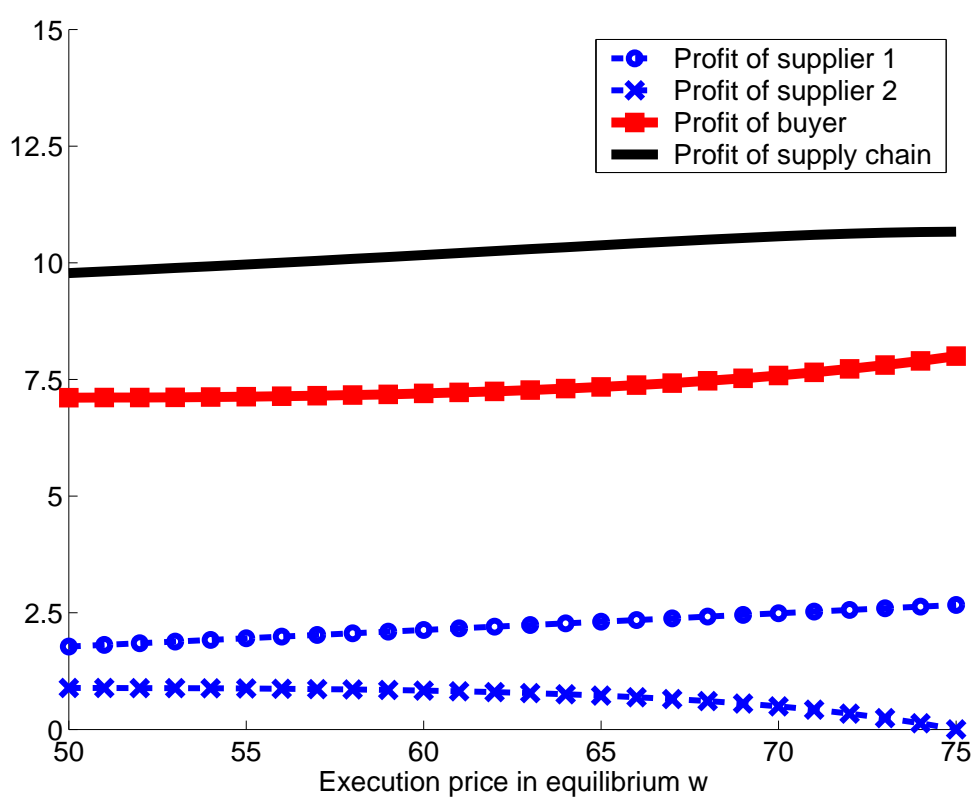

Figure 6: Expected profit of suppliers, buyer and the entire supply chain, for each possible equilibrium of the bidding game, as a function of $w$ (execution price of both suppliers in equilibrium), from Example 1.

The payments between buyer and suppliers will cancel out, and this quantity will only capture the true revenue from customers minus the costs of production. Thus, we can express the total supply chain surplus as

$$
\begin{aligned}
U & =p \int_{0}^{y_{N}} \bar{F}(u) d u-\sum_{i=1}^{N} f_{i}\left(y_{i}-y_{i-1}\right)-\sum_{i=1}^{N} c_{i} \int_{y_{i-1}}^{y_{i}} \bar{F}(u) d u \\
& =\sum_{i=1}^{N} \Delta c_{i} \int_{0}^{y_{i}}\left[\bar{F}(u)-\bar{F}\left(y_{i}^{*}\right)\right] d u .
\end{aligned}
$$

where $\bar{F}\left(y_{i}^{*}\right)=\frac{f_{i}-f_{i+1}}{c_{i+1}-c_{i}}$ and $\Delta c_{i}=c_{i+1}-c_{i}$. These quantities are well-defined when all the suppliers are efficient. The social surplus is maximized when $y_{i}=y_{i}^{*}, i=1, \ldots, N$. In this case, the optimal surplus is

$$
U^{*}=\sum_{i=1}^{N} \Delta c_{i} \int_{0}^{y_{i}^{*}}\left[\bar{F}(u)-\bar{F}\left(y_{i}^{*}\right)\right] d u .
$$

When the suppliers compete, the allocation of capacities, $y_{i}, i=1, \ldots, N$, is not necessarily efficient, in the sense that it is possible that $y_{i} \neq y_{i}^{*}$ for some $i$. The loss in surplus, due to the suppliers' competition, is equal to

$$
\Delta U=\sum_{i=1}^{N} \Delta c_{i} \int_{y_{i}}^{y_{i}^{*}}\left[\bar{F}(u)-\bar{F}\left(y_{i}^{*}\right)\right] d u .
$$


Theorem 4 Given a border demand distribution and efficient suppliers, in every equilibrium, the allocation of capacities obtains at least $50 \%$ of the optimal total surplus, i.e.,

$$
\frac{\Delta U}{U^{*}} \leq \frac{1}{2}
$$

This is the best bound available for general border distributions. However, this bound can be improved when we include additional conditions on the demand distribution, as shown next.

Theorem 5 Given a log-concave demand distribution and efficient suppliers, in every equilibrium, the allocation of capacities obtains at least $75 \%$ of the optimal total surplus, i.e.,

$$
\frac{\Delta U}{U^{*}} \leq \frac{1}{4}
$$

This bound is tight for two suppliers and uniform demand distribution.

The theorem thus implies that for uniform, exponential or normal demand distributions (which belong to the log-concave class) the loss of efficiency due to competition is no more than $25 \%$.

Interestingly, the loss of efficiency is due exclusively to supplier-supplier interactions, since, if all suppliers were integrated (or colluded), they would be able to extract all the supply chain profit ${ }^{8}$.

\subsection{Equilibria with inefficient suppliers}

The previous results, characterizing equilibrium, are obtained under the assumption that all suppliers are efficient. We now investigate the case in which not all suppliers are efficient.

Interestingly, as we demonstrate below, it might happen that a non-efficient supplier is active at equilibrium. This occurs because bids are only partially linked to the true costs, and a non-efficient supplier may capture market share by positioning itself in a segment of the market with no, or low, competition.

Example 2 Assume that customer demand is uniformly distributed in $[0,1]$. Let $N=3$ and the true costs be

$$
\left(c_{1}, f_{1}\right)=(0,40), \quad\left(c_{2}, f_{2}\right)=(40,20), \quad\left(c_{3}, f_{3}\right)=(70,11), \quad p=100 .
$$

Clearly, supplier 3 is not efficient. If this was a centralized system, in which the true costs are considered, we would have $y_{1}^{*}=0.5, y_{2}^{*}=0.666$ and $y_{3}^{*}=0.666$, and so the buyer would purchase capacities $x_{1}^{*}=0.5, x_{2}^{*}=0.166$ and $x_{3}^{*}=0$.

The following bids form an equilibrium:

$$
\left(w_{1}, v_{1}\right)=\left(w_{2}, v_{2}\right)=(20,30), \quad\left(w_{3}, v_{3}\right)=(100,0), \quad y_{1}=0.5, \quad y_{2}=0.625, \quad y_{3}=0.633 .
$$

Thus, a non-efficient supplier captures capacity and makes positive profit.

\footnotetext{
${ }^{8}$ For example, they could offer the contracts $w_{n}=p-\epsilon$ and $v_{n}=\bar{F}\left(y_{n}^{*}\right) \epsilon$, and for $i<n, w_{i}=w_{i+1}-\epsilon, v_{i}=v_{i+1}+\bar{F}\left(y_{i}^{*}\right) \epsilon$, for $\epsilon$ arbitrarily close to zero.
} 
The example suggests that the presence of inefficient suppliers can lead to counter-intuitive situations.

The next theorem depicts the behavior of the suppliers at equilibrium.

Theorem 6 For a border distribution, let $\left\{\left(w_{1}, v_{1}\right), \ldots,\left(w_{N}, v_{N}\right),(p, 0)\right\}$ be the bids of the suppliers in a equilibrium. Assume that supplier $i$ is active. Then we must have that:

- either there is $j=1, \ldots, N+1$ such that supplier $j$ is active, $\left(w_{i}, v_{i}\right)=\left(w_{j}, v_{j}\right)$ and moreover $\left(w_{i}, v_{i}\right)$ belongs in the segment $\left[\left(c_{i}, f_{i}\right) ;\left(c_{j}, f_{j}\right)\right]$;

- or there are $j, k=1, \ldots, N+1$ such that supplier $k$ is inactive, supplier $j$ is active and $\left(w_{i}, v_{i}\right)=\left(w_{k}, v_{k}\right)+\theta\left(w_{k}-w_{j}, v_{k}-v_{j}\right)$ for some $\theta \geq 0$.

This theorem adds a new case to what was presented in Theorem 3. This new situation arises when an inactive supplier sets the price of some active supplier: the reaction of the active supplier keeps the inactive (and inefficient) supplier out of the market by making its entry non-profitable.

Another example of this phenomenon can be found in the Bertrand model with asymmetric players. Although it is commonly argued that the only equilibrium in pure strategies is such that the most competitive producer captures all the market at a price equal to the second most competitive cost, as in Tirole [19] p.211, this equilibrium is not unique. As noted by Erlei [8], all the prices between the smallest and the second smallest costs are Nash equilibria of the system. This is true since an inefficient player can impact the market price by placing absurd bids knowing that it will not capture any market share. This is illustrated by the next example.

Example 3 Assume that customer demand is uniformly distributed in $[0,1]$. Let $N=4$ and the true costs be

$$
\left(c_{1}, f_{1}\right)=(0,40), \quad\left(c_{2}, f_{2}\right)=(40,20), \quad\left(c_{3}, f_{3}\right)=(70,6), \quad\left(c_{4}, f_{4}\right)=(80,6), \quad p=100 .
$$

Supplier 4 is not efficient, the rest are. The following bids form an equilibrium

$$
\begin{array}{cc}
\left(w_{1}, v_{1}\right)=\left(w_{2}, v_{2}\right)=(20,30), & \left(w_{3}, v_{3}\right)=\left(w_{4}, v_{4}\right)=(80,4) \\
y_{1}=0.5, y_{2}=0.567, & y_{3}=0.8, y_{4}=0.8 .
\end{array}
$$

Supplier 4, by placing a bid with which it would never make a positive profit, sets the price of supplier 3, who is efficient and must react to the threat of supplier 4.

Similarly to the Bertrand model with asymmetric players, it may be possible to discard some of the equilibria proposed by Theorem 6 , by eliminating for instance dominated strategies. Indeed, in Example 3, supplier 4 is bidding below cost, which is dominated by the strategy of bidding exactly the cost. 


\section{Discussion}

In this research, we have analyzed the procurement process between a single buyer and multiple suppliers. Suppliers compete on price and flexibility, two attributes that are important to the buyer. Specifically, each supplier is offering a different option contract and the buyer reserves capacities at each supplier so as to maximize expected profit. We have modeled the process as a single-shot game where the suppliers submit an offer with a reservation and an execution fee.

Under the assumption of the demand distribution having the border property, satisfied for instance by any log-concave distribution, e.g., uniform, exponential or normal distributions, we characterize optimality conditions for suppliers' bids and provide necessary conditions for equilibrium bids.

Interestingly, equilibria in pure strategies give rise to what we call cluster competition. This provides several insights.

1 It pays to be efficient. No matter how the competitors bid, when a supplier is efficient, it will capture orders from the buyer and will have a non-negative expected profit.

In other words, being an efficient supplier means capturing market share, and no other supplier can push an efficient supplier out of business. Notice that our definition of efficiency allows having multiple efficient technologies, because the cost space is two-dimensional. This implies that an inefficient supplier may become efficient by reaching the efficient frontier defined by the lower envelope of the true costs of the other suppliers. Hence, this inefficient supplier does not necessarily have to change technology and copy the same exact cost as other suppliers; what is needed is a local improvement of its costs so as to move to the efficient frontier.

2 Suppliers compete with suppliers with similar cost structure. When all suppliers are efficient, a supplier will compete against another supplier with similar technology, either the one with next lower or next higher execution cost.

Indeed, in equilibrium, a supplier's bid is most sensitive to the bid of another supplier with similar technology. This leads to our third insight.

3 Competition preserves diversity and segments the market. At a market equilibrium with efficient suppliers, the suppliers are clustered into small groups of no more than three suppliers and no less than two suppliers. All suppliers within each group offer the same option and share the order from the buyer.

The market will thus be segmented by groups of similar technologies. Competition will diminish technological variety but will not eliminate it. This is in contrast to market behavior in the priceonly competition. Thus, in our model, if at some point a supplier "kills" its competitors in a given 
niche, i.e., a given cluster, and its competitors exit the market, this supplier will increase its market share by moving to a different niche.

4 Prices are directly related to true cost. The equilibrium prices of the different options offered by the suppliers lie in the lower envelope of the costs of the system. That is, the reservation and execution equilibrium prices are linked to the true reservation and execution costs and no inflation of prices is stable.

This insight shows the link between the costs of the system and the option prices available in the market. Specifically, if all suppliers are efficient, this implies a range of possible bids, each of which is along the lower envelope of the true suppliers' costs. However, many equilibria are possible, and hence it is not possible to predict the option prices.

5 Competition leads to a loss of supply chain profit. While suppliers' prices are related to their true costs, the allocation of capacity can be quite different from the one achieved in a centralized system. However, our analysis indicates that the loss of system profit is no more than $50 \%$ of the maximum possible, and $25 \%$ for the class of log-concave distributions, a class that includes commonly used distributions such as the normal, uniform and exponential.

Finally, this paper will be incomplete if we do not mention important extensions of our model. One possible direction is to allow buyers to purchase products at a spot market in addition to using the contracts signed with the suppliers. In such a model, suppliers and buyers negotiate contracts knowing that additional supply or demand are available in the spot market. Such a model would generalize not only the model in the current paper but also the models presented in Wu et al.[21]. Another extension is to develop a multi-attribute competition for other factors such as quality or lead time, where the optimal portfolio for the buyer would be found endogenously. All these extensions present significant technical challenges.

\section{References}

[1] Barnes-Schuster D., Y. Bassok and R. Anupindi 2002. "Coordination and Flexibility in Supply Contracts with Options." Manufacturing \& Service Operations Management, 4(3), pp.171-207.

[2] Beil D. and L.M. Wein 2002. "An Inverse-Optimization-Based Auction Mechanism to Support a Multiattribute RFQ Process." Management Science, 49(11), pp. 1529-1545.

[3] Bertsekas D.P. 1995. Nonlinear Programming. Athena Scientific, Belmont, Massachusetts.

[4] Cachon G.P. 2002. "Supply Coordination with Contracts." forthcoming in Handbooks in Operations Research and Management Science, edited by Steve Graves and Ton de Kok, published by North-Holland. 
[5] Che Y.K. 1993. "Design Competition through Multidimensional Auctions." RAND Journal of Economics, 24(4), pp. 668-680.

[6] Dasgupta P. and E. Maskin 1986. "The Existence of Equilibrium in Discontinuous Economic Games, I: Theory." The Review of Economic Studies, 53(1), pp. 1-26.

[7] Eppen G. and A. Iyer 1997. "Backup Agreements in Fashion Buying - The Value of Upstream Flexibility." Management Science, 43(11), pp. 1469-1484.

[8] Erlei M. "Some Forgotten Equilibria of the Bertrand Duopoly." Note.

[9] Fudenberg D. and D. Levine 1986. "Limit Games and Limit Equilibria." Journal of Economic Theory, 38, pp. 251-268.

[10] Golovachkina N. and J. R. Bradley 2002. "Supplier-Manufacturer Relationships Under Forced Compliance Contracts." Working Paper, Cornell University.

[11] Lariviere M. 1999. "Supply Chain Contracting and Coordination with Stochastic Demand." S. Tayur, R. Ganeshan and M. Magazine, eds. Quantitative Models for Supply Chain Management, Kluwer Academic Publisher, Boston.

[12] Martínez-de-Albéniz V. 2004. "Portfolio Strategies in Supply Contracts." Ph.D. dissertation, OR Center, MIT.

[13] Martínez-de-Albéniz V. and D. Simchi-Levi 2005. "A Portfolio Approach to Procurement Contracts." Production and Operations Management, 14(1), pp. 90-114.

[14] Myerson R. 1981. "Optimal Auction Design." Mathematics of Operations Research, 6, pp. 58-73.

[15] Pasternack B. A. 1985. "Optimal Pricing and Returns Policies for Perishable Commodities." Marketing Science, 4(2), pp. 166-176.

[16] Radner R. 1980. "Collusive Behavior in Non-Cooperative Epsilon Equilibria of Oligopolies with Long but Finite Lives." Journal of Economic Theory, 22, pp. 136-154.

[17] Schummer J. and R.V. Vohra 2003. "Auctions for Procuring Options." Operations Research, 51(1), pp. 41-51.

[18] Spinler S., A. Huchzermeier, and P. R. Kleindorfer 2002. "The Valuation of Options on Capacity." Working paper, the Wharton School, University of Pennsylvania.

[19] Tirole J. 1988. The Theory of Industrial Organization. MIT Press, Cambridge, Massachusetts.

[20] Wu D.J. and P.R. Kleindorfer 2005. "Competitive Options, Supply Contracting, and Electronic Markets" Management Science, 51(3), pp. 452-466.

[21] Wu D.J., P.R. Kleindorfer, and Y. Sun 2005. "Optimal Capacity Expansion in the Presence of Capacity Options." Decision Support Systems, 40(3), pp. 553-561. 
[22] Wu D.J., P.R. Kleindorfer, and J.E. Zhang 2002. "Optimal Bidding and Contracting Strategies for Capital-Intensive Goods." European Journal of Operations Research, 137(3), pp. 657-676. 


\section{Competition in the Supply Option Market}

Victor Martínez-de-Albéniz and David Simchi-Levi

\section{Appendix: Proofs}

\section{Proposition 1}

Proof. To obtain the optimal portfolio we maximize function $V(\cdot)$ over the feasible region $P$ defined in Equation (3). From Equation (2), we observe that function $V(\cdot)$ is the sum of strictly concave functions of $y_{i}, i=1, \ldots, N$. Hence, it is strictly concave jointly in $\left(y_{1}, \ldots, y_{N}\right)$. The feasible region is a polyhedral cone with non-empty interior. This implies that the Slater conditions hold for this problem and that the Karush-Kuhn-Tucker conditions are necessary and sufficient at optimality (see Bertsekas [3] for details).

Define for every constraint $y_{i-1}-y_{i} \leq 0, i=1, \ldots, N$, the associate Lagrange multiplier $\lambda_{i} \geq 0$. The KKT optimality conditions are, for $i=1, \ldots, N$, assuming $\lambda_{N+1}=0$ :

$$
\begin{gathered}
\left(v_{i+1}-v_{i}\right)+\left(w_{i+1}-w_{i}\right) \bar{F}\left(y_{i}\right)=\lambda_{i+1}-\lambda_{i} \\
\lambda_{i}\left(y_{i-1}-y_{i}\right)=0 \\
y_{i-1}-y_{i} \leq 0 \\
\lambda_{i} \geq 0
\end{gathered}
$$

Let $\left\{i_{1}, \ldots, i_{k}\right\}$ be the winning set of $\left\{\left(w_{1}, v_{1}\right), \ldots,\left(w_{N+1}, v_{N+1}\right)\right\}$. Define $y_{i_{1}}, \ldots, y_{i_{k-1}}$ such that

$$
\bar{F}\left(y_{i_{j}}\right)=\frac{v_{i_{j}}-v_{i_{j+1}}}{w_{i_{j+1}}-w_{i_{j}}}
$$

$\bar{F}\left(y_{i_{k}}\right)=0$ and for the other variables $y_{i}=y_{i-1}$ (remember from Equation (1) that $\left.y_{0}=0\right)$. Note that it can happen that $y_{i}=\infty$ for some $i$. Define also $\lambda_{i_{1}}=\ldots=\lambda_{i_{k}}=0$ together with:

(i) for $1 \leq i<i_{1}, \lambda_{i}=\left(v_{i}-v_{i_{1}}\right)+\left(w_{i}-w_{i_{1}}\right)$,

(ii) for $j=1, \ldots, k-1$, for $i_{j}<i<i_{j+1}, \lambda_{i}=\left(v_{i}-v_{i_{j}}\right)+\left(w_{i}-w_{i_{j}}\right) \bar{F}\left(y_{i_{j}}\right)$,

(iii) for $i_{k}<i, \lambda_{i}=\left(v_{i}-v_{i_{k}}\right)$.

It is now sufficient to verify that this solution satisfies the KKT conditions, Equation (18). Evidently, the first three requirements in (18) are satisfied by construction. It remains to verify that $\lambda_{i} \geq 0$ for all $i=1, \ldots, N$. To see this, we analyze three different cases:

(i) for $1 \leq i<i_{1}, \lambda_{i}=\left(v_{i}-v_{i_{1}}\right)+\left(w_{i}-w_{i_{1}}\right) \geq 0$ from part (b) of Definition 2;

(ii) for $j=1, \ldots, k-1$, for $i_{j}<i<i_{j+1}, \lambda_{i}=\left(v_{i}-v_{i_{j}}\right)+\left(w_{i}-w_{i_{j}}\right) \frac{v_{i_{j}}-v_{i_{j+1}}}{w_{i_{j+1}}-w_{i_{j}}} \geq 0$ from part (c);

(iii) for $i_{k}<i, \lambda_{i}=\left(v_{i}-v_{i_{k}}\right) \geq 0$ from part (d). 
Finally, we see that no inactive point can be winning since this would imply that one of the inactive points is on the segment joining two other points. This would contradict the minimality of the winning set in Definition 2.

\section{Proof of Theorem 1}

Proof. It is easy to see that, in order to prove the result, one can show that the profit, as a function of $\left(y_{-}, y_{+}\right)$, does not achieve a local interior maximum, and this holds for all cost and rival bids parameters. For this purpose, assume that for a given set of parameters, we have a strict local maximum of the profit function.

Take $y_{m} \geq 0$, define

$$
\begin{aligned}
\alpha_{1} & =\frac{v^{l}-f}{c-w^{l}}, \\
\alpha_{2} & =\frac{f-v^{h}}{w^{h}-c},
\end{aligned}
$$

and $\alpha_{m}=\bar{F}\left(y_{m}\right)$. Assume, with no loss of generality, that the feasible region is $0 \leq y_{-} \leq y_{m} \leq y_{+}$. Let $\left(y_{-}, y_{+}\right)$be a strict local maximum of the function

$$
\Phi\left(y_{-}, y_{+}\right)=\frac{\alpha_{1}-\alpha_{m}}{\alpha_{1}-\alpha_{2}} \int_{y_{-}}^{y_{+}}\left(\bar{F}(u)-\alpha_{2}\right) d u-\frac{\bar{F}\left(y_{-}\right)-\alpha_{m}}{\bar{F}\left(y_{-}\right)-\bar{F}\left(y_{+}\right)} \int_{y_{-}}^{y_{+}}\left(\bar{F}(u)-\bar{F}\left(y_{+}\right)\right) d u,
$$

which corresponds to the profit divided by $w_{h}-w_{l}$. Let $\alpha_{-}=\bar{F}\left(y_{-}\right)$and $\alpha_{+}=\bar{F}\left(y_{+}\right)$.

Since this is a strict interior maximum, the first order conditions are, after recombining the different terms,

$$
\begin{aligned}
& 0=\frac{d \Phi}{d y_{-}}=-\frac{\left(\alpha_{m}-\alpha_{2}\right)\left(\alpha_{1}-\alpha_{-}\right)}{\left(\alpha_{1}-\alpha_{2}\right)}+f\left(y_{-}\right) \frac{\left(\alpha_{m}-\alpha_{+}\right)}{\left(\alpha_{-}-\alpha_{+}\right)^{2}}\left\{\int_{y_{-}}^{y_{+}}\left(\bar{F}(u)-\alpha_{+}\right) d u\right\} \\
& 0=\frac{d \Phi}{d y_{+}}=\frac{\left(\alpha_{1}-\alpha_{m}\right)\left(\alpha_{+}-\alpha_{2}\right)}{\left(\alpha_{1}-\alpha_{2}\right)}+f\left(y_{+}\right) \frac{\left(\alpha_{-}-\alpha_{m}\right)}{\left(\alpha_{-}-\alpha_{+}\right)^{2}}\left\{\int_{y_{-}}^{y_{+}}\left(\alpha_{-}-\bar{F}(u)\right) d u\right\} .
\end{aligned}
$$

Let

$$
A=\left(\frac{f(x)}{\bar{F}(x)-\bar{F}(y)}\right)\left(\frac{\int_{x}^{y}(\bar{F}(u)-\bar{F}(y)) d u}{\bar{F}(x)-\bar{F}(y)}\right)
$$

and

$$
B=\left(\frac{f(y)}{\bar{F}(x)-\bar{F}(y)}\right)\left(\frac{\int_{x}^{y}(\bar{F}(x)-\bar{F}(u)) d u}{\bar{F}(x)-\bar{F}(y)}\right) .
$$

The first order conditions are equivalent to

$$
\begin{aligned}
& A=\frac{\left(\alpha_{m}-\alpha_{2}\right)\left(\alpha_{1}-\alpha_{-}\right)}{\left(\alpha_{1}-\alpha_{2}\right)\left(\alpha_{m}-\alpha_{+}\right)}, \\
& B=\frac{\left(\alpha_{1}-\alpha_{m}\right)\left(\alpha_{+}-\alpha_{2}\right)}{\left(\alpha_{1}-\alpha_{2}\right)\left(\alpha_{-}-\alpha_{m}\right)},
\end{aligned}
$$


or equivalently,

$$
\begin{aligned}
& {\left[\frac{\alpha_{-}-\alpha_{+}}{\alpha_{1}-\alpha_{-}}-\frac{1-A}{A}\right]\left[\frac{\alpha_{-}-\alpha_{+}}{\alpha_{+}-\alpha_{2}}-\frac{1-B}{B}+\frac{1}{B}\right]=1+\frac{1}{A} \frac{\alpha_{-}-\alpha_{m}}{\alpha_{m}-\alpha_{+}},} \\
& {\left[\frac{\alpha_{-}-\alpha_{+}}{\alpha_{+}-\alpha_{2}}-\frac{1-B}{B}\right]\left[\frac{\alpha_{-}-\alpha_{+}}{\alpha_{1}-\alpha_{-}}-\frac{1-A}{A}+\frac{1}{A}\right]=1+\frac{1}{B} \frac{\alpha_{m}-\alpha_{+}}{\alpha_{-}-\alpha_{m}} .}
\end{aligned}
$$

We can see that when $f$ is log-concave, then

$$
\int_{x}^{y}(\bar{F}(x)-\bar{F}(u)) d u
$$

is log-concave in $y$. This implies that $0 \leq B \leq 1$. Similarly, $0 \leq A \leq 1$ when $f$ is log-concave. Thus under this assumption, $\alpha_{-} \geq \alpha_{m} \geq \alpha_{+}$implies that the first order conditions can only be satisfied when $\alpha_{+}-\alpha_{2} \geq 0$ and $\alpha_{1}-\alpha_{-} \geq 0$ or $\alpha_{+}-\alpha_{2} \leq 0$ and $\alpha_{1}-\alpha_{-} \leq 0$.

Let

$$
\begin{gathered}
a=\frac{\alpha_{-}-\alpha_{+}}{\alpha_{1}-\alpha_{-}}-\frac{1-A}{A} \\
b=\frac{\alpha_{-}-\alpha_{+}}{\alpha_{+}-\alpha_{2}}-\frac{1-B}{B} \\
c=\frac{\alpha_{-}-\alpha_{m}}{\alpha_{m}-\alpha_{+}} .
\end{gathered}
$$

Equation (21) can thus be expressed as

$$
\begin{aligned}
& a\left(b+\frac{1}{B}\right)-1=\frac{1}{A} c, \\
& b\left(a+\frac{1}{A}\right)-1=\frac{1}{B} \frac{1}{c} .
\end{aligned}
$$

By multiplying these two equations, one obtains

$$
\left[a\left(b+\frac{1}{B}\right)-1\right]\left[b\left(a+\frac{1}{A}\right)-1\right]=\frac{1}{A B},
$$

or equivalently

$$
[a b-1]\left[a b+\frac{a}{B}+\frac{b}{A}+\frac{1}{A B}-1\right]=0 .
$$

We have two possible cases:

1. In the first case, we have $a, b \geq 0, a b=1$, and thus $\alpha_{1} \geq \alpha_{-} \geq \alpha_{m} \geq \alpha_{+} \geq \alpha_{2}$. Thus Equation (21) becomes

$$
\begin{aligned}
& \frac{\alpha_{-}-\alpha_{+}}{\alpha_{1}-\alpha_{-}}-\frac{1-A}{A}=\frac{B}{A}\left[\frac{\alpha_{-}-\alpha_{m}}{\alpha_{m}-\alpha_{+}}\right] \\
& \frac{\alpha_{-}-\alpha_{+}}{\alpha_{+}-\alpha_{2}}-\frac{1-B}{B}=\frac{A}{B}\left[\frac{\alpha_{m}-\alpha_{+}}{\alpha_{-}-\alpha_{m}}\right] .
\end{aligned}
$$

2. In the second case, we have $a, b \leq 0$, and $a b=1-\frac{a}{B}-\frac{b}{A}-\frac{1}{A B}$. Thus, Equation (21) becomes

$$
\begin{aligned}
-\frac{\alpha_{-}-\alpha_{+}}{\alpha_{1}-\alpha_{-}}-1 & =\frac{\alpha_{m}-\alpha_{+}}{\alpha_{-}-\alpha_{m}}, \\
-\frac{\alpha_{-}-\alpha_{+}}{\alpha_{+}-\alpha_{2}}-1 & =\frac{\alpha_{-}-\alpha_{m}}{\alpha_{m}-\alpha_{+}} .
\end{aligned}
$$


The second order condition for having an interior local maximum is that the Hessian of $\Phi$ is negative semi-definite. It is straightforward to see that the Hessian being negative semi-definite is equivalent to having that $H$, defined as follows, is negative semi-definite.

$$
H=\left(\begin{array}{cc}
\frac{1}{A} \frac{d A}{d y_{-}}+\frac{f\left(y_{-}\right)}{\alpha_{1}-\alpha_{-}} & \frac{1}{A} \frac{d A}{d y_{+}}+\frac{f\left(y_{+}\right)}{\alpha_{m}-\alpha_{+}} \\
-\frac{1}{B} \frac{d B}{d y_{-}}+\frac{f\left(y_{-}\right)}{\alpha_{-}-\alpha_{m}} & -\frac{1}{B} \frac{d B}{d y_{+}}-\frac{f\left(y_{+}\right)}{\alpha_{+}-\alpha_{2}}
\end{array}\right)
$$

We compute the quantities that define $H$ in the following equations, evaluated at $\left(y_{-}, y_{+}\right)$. We have

$$
\begin{aligned}
\frac{1}{A} \frac{d A}{d y_{-}} & =\frac{f^{\prime}\left(y_{-}\right)}{f\left(y_{-}\right)}+\left[\frac{f\left(y_{-}\right)}{\bar{F}\left(y_{-}\right)-\bar{F}\left(y_{+}\right)}\right]\left[2-\frac{1}{A}\right], \\
\frac{1}{A} \frac{d A}{d y_{+}} & =\left[\frac{f\left(y_{+}\right)}{\bar{F}\left(y_{-}\right)-\bar{F}\left(y_{+}\right)}\right]\left[1+\frac{f\left(y_{-}\right) B}{f\left(y_{+}\right) A}\right]-2\left[\frac{f\left(y_{+}\right)}{\bar{F}\left(y_{-}\right)-\bar{F}\left(y_{+}\right)}\right] \\
\frac{1}{B} \frac{d B}{d y_{-}} & =-\left[\frac{f\left(y_{-}\right)}{\bar{F}\left(y_{-}\right)-\bar{F}\left(y_{+}\right)}\right]\left[1+\frac{f\left(y_{+}\right) A}{f\left(y_{-}\right) B}\right]+2\left[\frac{f\left(y_{-}\right)}{\bar{F}\left(y_{-}\right)-\bar{F}\left(y_{+}\right)}\right], \\
\frac{1}{B} \frac{d B}{d y_{+}} & =\frac{f^{\prime}\left(y_{+}\right)}{f\left(y_{+}\right)}-\left[\frac{f\left(y_{+}\right)}{\bar{F}\left(y_{-}\right)-\bar{F}\left(y_{+}\right)}\right]\left[2-\frac{1}{B}\right] .
\end{aligned}
$$

Thus, $H$ can be expressed as

$$
\begin{aligned}
H= & \left(\begin{array}{cc}
\frac{f^{\prime}\left(y_{-}\right)}{f\left(y_{-}\right)} & 0 \\
0 & -\frac{f^{\prime}\left(y_{+}\right)}{f\left(y_{+}\right)}
\end{array}\right) \\
& +\left(\begin{array}{cc}
{\left[\frac{f\left(y_{-}\right)}{\alpha_{-}-\alpha_{+}}\right]\left[2-\frac{1}{A}-\frac{\alpha_{-}-\alpha_{+}}{\alpha_{1}-\alpha_{-}}\right]} & \frac{B}{A}\left[\frac{f\left(y_{-}\right)}{\alpha_{-}-\alpha_{+}}\right]+\left[\frac{f\left(y_{+}\right)}{\alpha_{-}-\alpha_{+}}\right]\left[\frac{\alpha_{-}-\alpha_{m}}{\alpha_{m}-\alpha_{+}}\right] \\
\frac{A}{B}\left[\frac{f\left(y_{+}\right)}{\alpha_{-}-\alpha_{+}}\right]+\left[\frac{f\left(y_{-}\right)}{\alpha_{-}-\alpha_{+}}\right]\left[\frac{\alpha_{m}-\alpha_{+}}{\alpha_{-}-\alpha_{m}}\right] & {\left[\frac{f\left(y_{+}\right)}{\alpha_{-}-\alpha_{+}}\right]\left[2-\frac{1}{B}-\frac{\alpha_{-}-\alpha_{+}}{\alpha_{+}-\alpha_{2}}\right]}
\end{array}\right)
\end{aligned}
$$

In case (2) defined above, we can take a look at $H_{11}$, using Equation (23):

$$
\frac{f^{\prime}\left(y_{-}\right)}{f\left(y_{-}\right)}+\left[\frac{f\left(y_{-}\right)}{\alpha_{-}-\alpha_{+}}\right]\left[2-\frac{1}{A}-\frac{\alpha_{-}-\alpha_{+}}{\alpha_{1}-\alpha_{-}}\right] \geq \frac{f^{\prime}\left(y_{-}\right)}{f\left(y_{-}\right)}+\left[\frac{f\left(y_{-}\right)}{\alpha_{-}-\alpha_{+}}\right]\left[3-\frac{1}{A}\right] .
$$

This quantity is the derivative of $\bar{A}=\frac{A}{\bar{F}\left(y_{-}\right)-\bar{F}\left(y_{+}\right)}$with respect to $y_{-}$. We have that

$$
\frac{1}{\bar{A}} \frac{d \bar{A}}{d y_{-}}=\left\{\begin{array}{l}
\frac{f^{\prime}\left(y_{-}\right)}{f\left(y_{-}\right)}+\frac{f\left(y_{-}\right)}{\bar{F}\left(y_{-}\right)-\bar{F}\left(y_{+}\right)} \\
+\left[\frac{f\left(y_{-}\right)}{\bar{F}\left(y_{-}\right)-\bar{F}\left(y_{+}\right)}\right]\left[2-\frac{1}{A}\right]
\end{array}\right.
$$

Since

$$
\frac{1}{A} \frac{d A}{d y_{-}}=\frac{f^{\prime}\left(y_{-}\right)}{f\left(y_{-}\right)}+\left[\frac{f\left(y_{-}\right)}{\bar{F}\left(y_{-}\right)-\bar{F}\left(y_{+}\right)}\right]\left[2-\frac{1}{A}\right]
$$


when $A$ is increasing at $y_{-}$, then $\bar{A}$ also is. On the other hand, when $A$ is decreasing at $y_{-}$, and since $A\left(y_{+}, y_{+}\right)=1 / 2$, it must be that $A \geq 1 / 2$. Log-concavity of $f$ implies that

$$
\frac{f^{\prime}\left(y_{-}\right)}{f\left(y_{-}\right)}+\frac{f\left(y_{-}\right)}{\bar{F}\left(y_{-}\right)-\bar{F}\left(y_{+}\right)} \geq 0
$$

and thus $\bar{A}$ is again non-decreasing in $y_{-}$. Thus, $\frac{A}{\bar{F}\left(y_{-}\right)-\bar{F}\left(y_{+}\right)}$is non-decreasing, which implies that $H_{11}$ is non-negative. The same is true for $H_{22}$. Thus, in case (2), the matrix $H$ cannot be negative semi-definite.

In case (1), using that $c=\frac{\alpha_{-}-\alpha_{m}}{\alpha_{m}-\alpha_{+}}$,

$$
\begin{aligned}
H= & \left(\begin{array}{cc}
\frac{f^{\prime}\left(y_{-}\right)}{f\left(y_{-}\right)} & 0 \\
0 & -\frac{f^{\prime}\left(y_{+}\right)}{f\left(y_{+}\right)}
\end{array}\right) \\
& +\left(\begin{array}{cc}
{\left[\frac{f\left(y_{-}\right)}{\alpha_{-}-\alpha_{+}}\right]\left[3-\frac{2}{A}-\frac{B c}{A}\right]} & \frac{B}{A}\left[\frac{f\left(y_{-}\right)}{\alpha_{-}-\alpha_{+}}\right]+c\left[\frac{f\left(y_{+}\right)}{\alpha_{-}-\alpha_{+}}\right] \\
\frac{A}{B}\left[\frac{f\left(y_{+}\right)}{\alpha_{-}-\alpha_{+}}\right]+\frac{1}{c}\left[\frac{f\left(y_{-}\right)}{\alpha_{-}-\alpha_{+}}\right] & {\left[\frac{f\left(y_{+}\right)}{\alpha_{-}-\alpha_{+}}\right]\left[3-\frac{2}{B}-\frac{A}{B c}\right]}
\end{array}\right)
\end{aligned}
$$

To get rid of $c$, we examine 


$$
\begin{aligned}
& \left(\begin{array}{ll}
\frac{A}{f\left(y_{-}\right)} & \frac{B}{f\left(y_{+}\right)}
\end{array}\right) H\left(\begin{array}{c}
\frac{A}{f\left(y_{-}\right)} \\
\frac{B}{f\left(y_{+}\right)}
\end{array}\right) \\
& =\left\{\begin{array}{l}
\frac{f^{\prime}\left(y_{-}\right)}{f\left(y_{-}\right)} \frac{A^{2}}{f\left(y_{-}\right)^{2}} \\
-\frac{f^{\prime}\left(y_{+}\right)}{f\left(y_{+}\right)} \frac{B^{2}}{f\left(y_{+}\right)^{2}} \\
+\frac{2 A(2 A-1)}{f\left(y_{-}\right)\left(\alpha_{-}-\alpha_{+}\right)}+\frac{2 B(2 B-1)}{f\left(y_{+}\right)\left(\alpha_{-}-\alpha_{+}\right)}
\end{array}\right. \\
& =\frac{1}{\left(\alpha_{-}-\alpha_{+}\right)^{2}}\left\{\begin{array}{l}
\frac{f^{\prime}\left(y_{-}\right)}{f\left(y_{-}\right)}\left\{\int_{y_{-}}^{y_{+}}\left(\frac{\bar{F}(u)-\alpha_{+}}{\alpha_{-}-\alpha_{+}}\right) d u\right\}^{2} \\
-\frac{f^{\prime}\left(y_{+}\right)}{f\left(y_{+}\right)}\left\{\int_{y_{-}}^{y_{+}}\left(\frac{\alpha_{-}-\bar{F}(u)}{\alpha_{-}-\alpha_{+}}\right) d u\right\}^{2} \\
+2(2 A-1)\left\{\int_{y_{-}}^{y_{+}}\left(\frac{\bar{F}(u)-\alpha_{+}}{\alpha_{-}-\alpha_{+}}\right) d u\right\} \\
+2(2 B-1)\left\{\int_{y_{-}}^{y_{+}}\left(\frac{\alpha_{-}-\bar{F}(u)}{\alpha_{-}-\alpha_{+}}\right) d u\right\}
\end{array}\right.
\end{aligned}
$$

Using that

$$
\Delta=y_{+}-y_{-}=\int_{y_{-}}^{y_{+}}\left(\frac{\bar{F}(u)-\alpha_{+}}{\alpha_{-}-\alpha_{+}}\right) d u+\int_{y_{-}}^{y_{+}}\left(\frac{\alpha_{-}-\bar{F}(u)}{\alpha_{-}-\alpha_{+}}\right) d u
$$

and defining

$$
z=\int_{y_{-}}^{y_{+}}\left(\frac{\bar{F}(u)-\alpha_{+}}{\alpha_{-}-\alpha_{+}}\right) d u
$$

we can express the terms in the last bracket as

$$
E=\frac{f^{\prime}\left(y_{-}\right)}{f\left(y_{-}\right)} z^{2}-\frac{f^{\prime}\left(y_{+}\right)}{f\left(y_{+}\right)}(\Delta-z)^{2}+4 \frac{f\left(y_{-}\right)}{\alpha_{-}-\alpha_{+}} z^{2}+4 \frac{f\left(y_{+}\right)}{\alpha_{-}-\alpha_{+}}(\Delta-z)^{2}-2 \Delta .
$$

By minimizing this expression in terms of $z$, we obtain a lower bound on this expression, i.e. 


$$
\begin{aligned}
E \geq & \Delta\left\{\frac{\left[\frac{f^{\prime}\left(y_{-}\right)}{f\left(y_{-}\right)}+4 \frac{f\left(y_{-}\right)}{\alpha_{-}-\alpha_{+}}\right]\left[-\frac{f^{\prime}\left(y_{+}\right)}{f\left(y_{+}\right)}+4 \frac{f\left(y_{+}\right)}{\alpha_{-}-\alpha_{+}}\right] \Delta}{\left[\frac{f^{\prime}\left(y_{-}\right)}{f\left(y_{-}\right)}+4 \frac{f\left(y_{-}\right)}{\alpha_{-}-\alpha_{+}}-\frac{f^{\prime}\left(y_{+}\right)}{f\left(y_{+}\right)}+4 \frac{f\left(y_{+}\right)}{\alpha_{-}-\alpha_{+}}\right]}-2\right\} \\
= & \left\{\frac{1}{f^{\prime}\left(y_{-}\right)}\right. \\
& \left\{\left[\frac{f\left(y_{-}\right)}{f\left(y_{-}\right)} \Delta+4 \frac{f\left(y_{-}\right)}{\alpha_{-}-\alpha_{+}}-\frac{f^{\prime}\left(y_{+}\right)}{f\left(y_{-}\right)}+4 \frac{f\left(y_{+}\right)}{\alpha_{-}-\alpha_{+}}-2\right]\left[-\frac{f^{\prime}\left(y_{+}\right)}{f\left(y_{+}\right)} \Delta+4 \frac{f\left(y_{+}\right) \Delta}{\alpha_{-}-\alpha_{+}}-2\right]-4\right\}
\end{aligned}
$$

We thus focus on the last term of the product,

$$
F=\left(\frac{f^{\prime}\left(y_{-}\right)}{f\left(y_{-}\right)} \Delta+4 \frac{f\left(y_{-}\right) \Delta}{\alpha_{-}-\alpha_{+}}-2\right)\left(-\frac{f^{\prime}\left(y_{+}\right)}{f\left(y_{+}\right)} \Delta+4 \frac{f\left(y_{+}\right) \Delta}{\alpha_{-}-\alpha_{+}}-2\right)-4 .
$$

Over all log-concave distribution functions, $F$ defined in Equation (25) is minimized when $\alpha_{-}-\alpha_{+}$ is maximized. This occurs when, after defining $\theta$ by $\beta_{-} \theta+\beta_{+}(1-\theta)=\beta_{0}$,

$$
f(t)= \begin{cases}f\left(y_{-}\right) e^{\beta_{-}\left(t-y_{-}\right) / \Delta} & \text { when } y_{-} \leq t \leq y_{-}+\theta \Delta \\ f\left(y_{+}\right) e^{-\beta_{+}\left(y_{+}-t\right) / \Delta} & \text { when } y_{+}-(1-\theta) \Delta \leq t \leq y_{+}\end{cases}
$$

We thus know the structure of the worst-case log-concave distribution. By re-scaling the problem, $F$ can be expressed using only $\beta_{-}, \beta_{+}$(with $\beta_{+} \leq \beta_{-}$) and $\theta \in[0,1]$. To obtain the following expression, we scale $\Delta$ to 1 and the break-point value of the distribution $f\left(y_{-}+\theta \Delta\right)$ to 1 . After defining, for $k \geq 0$,

$$
P_{k}(z)=\frac{e^{z}-1-z-\ldots-z^{k-1} /(k-1) !}{z^{k}}
$$

we obtained the following scaled quantities

$$
\begin{aligned}
& f\left(y_{-}\right)=e^{-\beta_{-} \theta}=P_{0}\left(-\beta_{-} \theta\right) \\
& f\left(y_{+}\right)=e^{\beta_{+}(1-\theta)}=P_{0}\left(\beta_{+}(1-\theta)\right) \\
& \alpha_{-}-\alpha_{+}=\frac{e^{-\beta_{-} \theta}-1}{-\beta_{-}}+\frac{e^{\beta_{+}(1-\theta)}-1}{\beta_{+}}=\theta P_{1}\left(-\beta_{-} \theta\right)+(1-\theta) P_{1}\left(\beta_{+}(1-\theta)\right) .
\end{aligned}
$$

Define

$$
\begin{aligned}
& R_{1}\left(\beta_{-}, \beta_{+}\right)=\frac{P_{0}\left(-\beta_{-} \theta\right)}{\theta P_{1}\left(-\beta_{-} \theta\right)+(1-\theta) P_{1}\left(\beta_{+}(1-\theta)\right)}, \\
& G_{1}\left(\beta_{-}, \beta_{+}\right)=\beta_{-}-2+4 R_{1}\left(\beta_{-}, \beta_{+}\right) \geq 0,
\end{aligned}
$$

and

$$
\begin{aligned}
& R_{2}\left(\beta_{-}, \beta_{+}\right)=\frac{P_{0}\left(\beta_{+}(1-\theta)\right)}{\theta P_{1}\left(-\beta_{-} \theta\right)+(1-\theta) P_{1}\left(\beta_{+}(1-\theta)\right)}, \\
& G_{2}\left(\beta_{-}, \beta_{+}\right)=-\beta_{+}-2+4 R_{2}\left(\beta_{-}, \beta_{+}\right) \geq 0 .
\end{aligned}
$$


Thus, $F$ can be expressed as $G_{1} G_{2}-4$.

Notice that, since

$$
P_{1}(z)^{\prime}=P_{1}(z)-P_{2}(z)=P_{0}(z) P_{2}(-z)
$$

we have that

$$
\begin{aligned}
& \frac{d R_{1}}{d \beta_{-}}=R_{1}\left(-\theta+\theta^{2} P_{2}\left(\beta_{-} \theta\right) R_{1}\right) \\
& \frac{d R_{1}}{d \beta_{+}}=R_{1}\left(-(1-\theta)^{2} P_{2}\left(-\beta_{+}(1-\theta)\right) R_{2}\right),
\end{aligned}
$$

and

$$
\begin{aligned}
& \frac{d R_{2}}{d \beta_{-}}=R_{2}\left(\theta^{2} P_{2}\left(\beta_{-} \theta\right) R_{1}\right) \\
& \frac{d R_{2}}{d \beta_{+}}=R_{2}\left((1-\theta)-(1-\theta)^{2} P_{2}\left(-\beta_{+}(1-\theta)\right) R_{2}\right),
\end{aligned}
$$

In order to obtain a lower bound on $F$, we examine two different cases: either the minimal value of $F$ subject to $\theta \in[0,1]$ and $\beta_{-} \geq \beta_{+}$is reached in an interior point, or it is reached at the border of the region, i.e., $\theta=0, \theta=1$ or $\beta_{-}=\beta_{+}$; in any of the latter cases, the distribution turns out to be an exponential distribution.

To analyze the first case, let's examine the critical points of $F$ with respect to $\theta, \beta_{-}$and $\beta_{+}$. That is, assume that

$$
\begin{aligned}
& \frac{d F}{d \theta}=0=-\left[4 R_{1}\left(\beta_{-}-\left(R_{2}-R_{1}\right)\right)\right] G_{2}+G_{1}\left[4 R_{2}\left(\left(R_{2}-R_{1}\right)-\beta_{+}\right)\right], \\
& \frac{d F}{d \beta_{-}}=0=\left[1+4 R_{1} \theta\left(-1+\theta P_{2}\left(\beta_{-} \theta\right) R_{1}\right)\right] G_{2}+G_{1}\left[4 R_{1} R_{2} \theta^{2} P_{2}\left(\beta_{-} \theta\right)\right],
\end{aligned}
$$

and

$$
\frac{d F}{d \beta_{+}}=0=\left[-4 R_{1} R_{2}(1-\theta)^{2} P_{2}\left(-\beta_{+}(1-\theta)\right)\right] G_{2}+G_{1}\left[-1+4 R_{2}(1-\theta)\left(1-(1-\theta) P_{2}\left(-\beta_{+}(1-\theta)\right) R_{2}\right)\right] .
$$

Notice that we can express

$$
\begin{aligned}
R_{2}-R_{1} & =\frac{P_{0}\left(\beta_{+}(1-\theta)\right)-P_{0}\left(-\beta_{-} \theta\right)}{\theta P_{1}\left(-\beta_{-} \theta\right)+(1-\theta) P_{1}\left(\beta_{+}(1-\theta)\right)} \\
& =\beta_{+}+\frac{\theta\left(\beta_{-}-\beta_{+}\right) P_{1}\left(-\beta_{-} \theta\right)}{\theta P_{1}\left(-\beta_{-} \theta\right)+(1-\theta) P_{1}\left(\beta_{+}(1-\theta)\right)} \\
& =\beta_{-}-\frac{(1-\theta)\left(\beta_{-}-\beta_{+}\right) P_{1}\left(\beta_{+}(1-\theta)\right)}{\theta P_{1}\left(-\beta_{-} \theta\right)+(1-\theta) P_{1}\left(\beta_{+}(1-\theta)\right)}
\end{aligned}
$$


Thus, Equation (27) can be rewritten as

$$
\frac{R_{1} / G_{1}}{R_{2} / G_{2}}=\frac{\left(R_{2}-R_{1}\right)-\beta_{+}}{\beta_{-}-\left(R_{2}-R_{2}\right)}=\frac{\theta}{1-\theta} \frac{P_{1}\left(-\beta_{-} \theta\right)}{P_{1}\left(\beta_{+}(1-\theta)\right)} .
$$

Equation (28) can be expressed as

$$
4 \theta \frac{R_{1}}{G_{1}}=\frac{1}{G_{1}}+4 \theta^{2} P_{2}\left(\beta \_\theta\right) R_{1}\left[\frac{R_{1}}{G_{1}}+\frac{R_{2}}{G_{2}}\right] .
$$

Using the inequality $(a+b)^{2} \geq 4 a b$ for any $a, b \in \mathbb{R}$, we obtain

$$
16 \theta^{2}\left[\frac{R_{1}}{G_{1}}\right]^{2} \geq 16 \theta^{2} P_{2}\left(\beta_{-} \theta\right) \frac{R_{1}}{G_{1}}\left[\frac{R_{1}}{G_{1}}+\frac{R_{2}}{G_{2}}\right],
$$

and hence

$$
\frac{R_{1}}{G_{1}} \geq P_{2}\left(\beta_{-} \theta\right)\left[\frac{R_{1}}{G_{1}}+\frac{R_{2}}{G_{2}}\right]
$$

Similarly, Equation (29) yields

$$
\frac{R_{2}}{G_{2}} \geq P_{2}\left(-\beta_{+}(1-\theta)\right)\left[\frac{R_{1}}{G_{1}}+\frac{R_{2}}{G_{2}}\right],
$$

Adding these two last equations, the term $R_{1} / G_{1}+R_{2} / G_{2}$ cancels out, and thus

$$
1 \geq P_{2}\left(\beta_{-} \theta\right)+P_{2}\left(-\beta_{+}(1-\theta)\right) .
$$

Notice that $P_{2}$ is convex, since $P_{2}^{\prime \prime}=P_{2}-4 P_{3}+6 P_{4} \geq 0$. We can therefore apply the convexity inequality

$$
\frac{1}{2} P_{2}\left(\beta_{-} \theta\right)+\frac{1}{2} P_{2}\left(-\beta_{+}(1-\theta)\right) \geq P_{2}\left(\frac{1}{2} \beta_{-} \theta-\frac{1}{2} \beta_{+}(1-\theta)\right) .
$$

Thus, since $P_{2}(z) \leq 1 / 2$ if and only if $z \leq 0$, combining the two inequalities, we obtain that

$$
\beta_{-} \theta \leq \beta_{+}(1-\theta)
$$

Also, since $\beta_{-} \geq \beta_{+}$by construction, we have that

$$
\beta_{-} \theta \leq \beta_{+}(1-\theta) \leq \beta_{-}(1-\theta)
$$

This implies, that for any critical point, we must have:

- either $\beta_{-} \geq \beta_{+} \geq 0$ and $\theta \leq \frac{1}{2}$;

- or $0 \geq \beta_{-} \geq \beta_{+}$and $\theta \geq \frac{1}{2}$.

When $\theta<1 / 2$, Equation (31) yields

$$
\frac{R_{1}}{G_{1}} \geq \frac{1}{2}\left[\frac{R_{1}}{G_{1}}+\frac{R_{2}}{G_{2}}\right],
$$


and thus

$$
\frac{R_{1}}{G_{1}} \geq \frac{R_{2}}{G_{2}}
$$

On the other hand, Equation (30) implies that, since $\theta /(1-\theta)<1, P_{1}\left(-\beta_{-} \theta\right) \leq P_{1}(0)=1$ and $P_{1}\left(\beta_{+}(1-\theta)\right) \geq P_{1}(0)=1$,

$$
\frac{R_{1} / G_{1}}{R_{2} / G_{2}}=\frac{\theta}{1-\theta} \frac{P_{1}\left(-\beta_{-} \theta\right)}{P_{1}\left(\beta_{+}(1-\theta)\right)}<1
$$

This is a contradiction.

Similarly, when $\theta>1 / 2$, we have again a contradiction using Equation (32) to show

$$
\frac{R_{1}}{G_{1}} \leq \frac{R_{2}}{G_{2}}
$$

and Equation (30) to show

$$
\frac{R_{1} / G_{1}}{R_{2} / G_{2}}>1
$$

Thus, the only feasible case is $\theta=1 / 2$ which implies $\beta_{-}=\beta_{+}=0$, and in this case $G_{1}=G_{2}=2$, so that $F \geq 0$.

Thus, there are no critical points in the interior of $\theta \in[0,1]$ and $\beta_{-} \geq \beta_{+}$such that $F<0$.

The remaining case is when the minimum of $F$ is reached at the border of the feasible set, i.e., the distribution is exponential, with parameter $\gamma$. In this case, we can express $F$ as $g(\gamma) g(-\gamma)-4$, with, for $z \in \mathbb{R}$,

$$
g(z)=z-2+\frac{4}{P_{1}(z)} .
$$

Note that $g(z)-g(-z)=-2 z$ and

$$
\begin{aligned}
g(z)+g(-z) & =4 \frac{z\left(1-e^{-z}\right)+z\left(e^{z}-1\right)-\left(e^{z}-1\right)\left(e^{-z}-1\right)}{\left(e^{z}-1\right)\left(e^{-z}-1\right)} \\
& =4\left\{\frac{P_{1}(-z)+P_{1}(z)}{P_{2}(-z)+P_{2}(z)}-1\right\} .
\end{aligned}
$$

Hence,

$$
\begin{aligned}
g(z) g(-z)-4 & =\frac{1}{4}(g(z)+g(-z))^{2}-\frac{1}{4}(g(z)-g(-z))^{2}-4 \\
& =4 \frac{\left\{P_{1}(-z)+P_{1}(z)\right\}^{2}}{\left\{P_{2}(-z)+P_{2}(z)\right\}^{2}}-8 \frac{\left\{P_{1}(-z)+P_{1}(z)\right\}}{\left\{P_{2}(-z)+P_{2}(z)\right\}}-z^{2}
\end{aligned}
$$

After putting all terms under a common denominator, and observing that

$$
\begin{aligned}
& \left\{\begin{array}{l}
\left.P_{1}(-z)+P_{1}(z)\right\}^{2}=4\left\{P_{2}(-2 z)+P_{2}(2 z)\right\}, \\
\left.P_{1}(-z)+P_{1}(z)\right\}\left\{P_{2}(-z)+P_{2}(z)\right\}=2\left\{P_{1}(-2 z)+P_{1}(2 z)-P_{1}(-z)-P_{1}(z)\right\} / z^{2},
\end{array}\right. \\
& \left\{P_{2}(-z)+P_{2}(z)\right\}^{2}=4\left\{P_{2}(-2 z)+P_{2}(2 z)-P_{2}(-z)-P_{2}(z)\right\},
\end{aligned}
$$


the numerator can be expressed as

$12\left\{P_{2}(-2 z)+P_{2}(2 z)\right\}+4\left\{P_{2}(-z)+P_{2}(z)\right\}-16\left\{P_{1}(-2 z)+P_{1}(2 z)\right\} / z^{2}+16\left\{P_{1}(-z)+P_{1}(z)\right\} / z^{2}$.

We can use a series expansion to show that this final term is non-negative:

$$
2 \sum_{k=0}^{\infty}\left\{\frac{12 \cdot 2^{2 k} z^{2 k}}{(2 k+2) !}+\frac{4 z^{2 k}}{(2 k+2) !}-\frac{16 \cdot 2^{2 k+2} z^{2 k}}{(2 k+3) !}+\frac{16 z^{2 k}}{(2 k+3) !}\right\}
$$

The coefficient of $z^{2 k}$ in the series is

$$
\frac{12(2 k+3) 4^{k}+4(2 k+3)-64 \cdot 4^{k}+16}{(2 k+3) !} \geq 0
$$

for all $k \geq 0$. This shows that $F \geq 0$, in all cases.

This completes the proof, since we have found that the second order maximality condition cannot be satisfied.

\section{Theorem 2}

Proof. We have explained previously that under the assumptions of the proposition, either $y_{+}^{*}=y_{m}$ and $y_{-}^{*}=y_{1}$ or $y_{+}^{*}=y_{2}$ and $y_{-}^{*}=y_{m}$. Otherwise, it would be optimal to bid in some other region $A_{l^{\prime} h^{\prime}}$ in addition to $A_{l h}$. Since this is a contradiction to the hypothesis, it implies that the two possible optimal bids are either $\left(w_{l}, v_{l}\right)$ or $\left(w_{h}, v_{h}\right)$.

If $y_{1}>y_{m}$ or $y_{2}<y_{m}$, from Equation (12) it is clear that it is not optimal for the supplier to bid in this particular region $A_{l h}$, because it has an incentive to bid in $A_{O U T}$ instead of $A_{l h}$. Similarly, if $y_{1}<y_{0}$ and $y_{2}>y_{3}$, neither one of the bids is admissible, and therefore there is an optimum outside $A_{l h}$. We can now partition the remaining possibilities into the three cases presented in the proposition.

In the two first cases, since only one of the two bids is admissible, it must be optimal. In the third case, it implies that $(c, f) \in A_{l h}$. Bidding $\left(w_{h}, v_{h}\right)$ is better than $\left(w_{l}, v_{l}\right)$ when

$$
\Pi_{2}=\left(v_{h}-f\right)\left(y_{2}-y_{m}\right)+\left(w_{h}-c\right) \int_{y_{m}}^{y_{2}} \bar{F}(u) d u \geq \Pi_{1}=\left(v_{l}-f\right)\left(y_{m}-y_{1}\right)+\left(w_{l}-c\right) \int_{y_{1}}^{y_{m}} \bar{F}(u) d u .
$$

Using Equations (14) and (15), this is equivalent to

$$
\left(w_{h}-c\right) \int_{y_{m}}^{y_{2}}\left[\bar{F}(u)-\bar{F}\left(y_{2}\right)\right] d u \geq\left(c-w_{l}\right) \int_{y_{1}}^{y_{m}}\left[\bar{F}\left(y_{1}\right)-\bar{F}(u)\right] d u .
$$

But also, we have that, similarly to Equation (9),

$$
c=w_{h}-\left(w_{h}-w_{l}\right) \frac{\bar{F}\left(y_{1}\right)-\bar{F}\left(y_{m}\right)}{\bar{F}\left(y_{1}\right)-\bar{F}\left(y_{2}\right)}=w_{l}+\left(w_{h}-w_{l}\right) \frac{\bar{F}\left(y_{m}\right)-\bar{F}\left(y_{2}\right)}{\bar{F}\left(y_{1}\right)-\bar{F}\left(y_{2}\right)} .
$$


Therefore, we can rewrite the previous condition as

$$
\frac{\bar{F}\left(y_{1}\right)-\bar{F}\left(y_{m}\right)}{\bar{F}\left(y_{1}\right)-\bar{F}\left(y_{2}\right)} \int_{y_{m}}^{y_{2}}\left[\bar{F}(u)-\bar{F}\left(y_{2}\right)\right] d u \geq \frac{\bar{F}\left(y_{m}\right)-\bar{F}\left(y_{2}\right)}{\bar{F}\left(y_{1}\right)-\bar{F}\left(y_{2}\right)} \int_{y_{1}}^{y_{m}}\left[\bar{F}\left(y_{1}\right)-\bar{F}(u)\right] d u .
$$

After simplifying this expression, we obtain Equations (16) and (17).

\section{Proposition 2}

Proof. Consider, in an equilibrium, that $i$ and $j$ submit the same bid $(w, v)$. That is, for each $\epsilon>0$, there are $\left(w_{i}^{\epsilon}, v_{i}^{\epsilon}\right),\left(w_{j}^{\epsilon}, v_{j}^{\epsilon}\right)$ that converge to $(w, v)$ and such that

$$
\Pi_{i}\left(w_{i}^{\epsilon}, v_{i}^{\epsilon}, \mathbf{w}_{-i}^{\epsilon}, \mathbf{v}_{-i}^{\epsilon}\right) \geq \sup _{(w, v)} \Pi_{i}\left(w, v, \mathbf{w}_{-i}^{\epsilon}, \mathbf{v}_{-i}^{\epsilon}\right)-\epsilon
$$

and

$$
\Pi_{j}\left(w_{j}^{\epsilon}, v_{j}^{\epsilon}, \mathbf{w}_{-j}^{\epsilon}, \mathbf{v}_{-j}^{\epsilon}\right) \geq \sup _{(w, v)} \Pi_{i}\left(w, v, \mathbf{w}_{-j}^{\epsilon}, \mathbf{v}_{-j}^{\epsilon}\right)-\epsilon .
$$

Since in the limit $\epsilon \rightarrow 0$, both suppliers are making positive profits, then it must be true that either (1) for $\epsilon$ sufficiently small, $\left(w_{i}^{\epsilon}, v_{i}^{\epsilon}\right)$ must be in some region $A_{l j}^{i}$; or (2) (1) for $\epsilon$ sufficiently small, $\left(w_{i}^{\epsilon}, v_{i}^{\epsilon}\right)$ must be in some region $A_{j h}^{i}$. Similarly, $\left(w_{j}^{\epsilon}, v_{j}^{\epsilon}\right)$ must be for $\epsilon$ small in some region $A_{l^{\prime} i}^{j}$ or $A_{i h^{\prime}}^{j}$. Indeed, if $i$ (resp. $j$ ) bids in a different region, then $i$ (resp. $j$ ) makes $j$ (resp. $i$ ) inactive, which would yield zero profit for one of the suppliers (this is ruled out by the assumption of the proposition).

We can now apply Theorem 2. Assume that $i$ bids in $A_{l j}^{i}$. Then $j$ must bid in $A_{i h^{\prime}}^{j}$. By using the optimality equations (14) and (15), $y_{i-}^{\epsilon} \approx y_{m}=\bar{F}^{-1}\left(\frac{v_{l}-v}{w-w_{l}}\right)$ and $y_{i+}^{\epsilon}=\bar{F}^{-1}\left(\frac{v_{i}^{\epsilon}-v_{j}^{\epsilon}}{w_{j}^{\epsilon}-w_{i}^{\epsilon}}\right) \approx$ $y_{i 2}=\bar{F}^{-1}\left(\frac{f_{i}-v}{w-c_{i}}\right)$, and hence we have that the slope between $\left(c_{i}, f_{i}\right)$ and $\left(w_{i}^{\epsilon}, v_{i}^{\epsilon}\right)$, and $\left(w_{i}^{\epsilon}, v_{i}^{\epsilon}\right)$ and $\left(w_{j}^{\epsilon}, v_{j}^{\epsilon}\right)$ must be very close. The same applies to the slope between $\left(c_{j}, f_{j}\right)$ and $\left(w_{j}^{\epsilon}, v_{j}^{\epsilon}\right)$, and $\left(w_{j}^{\epsilon}, v_{j}^{\epsilon}\right)$ and $\left(w_{i}^{\epsilon}, v_{i}^{\epsilon}\right)$. Hence, taking the limit as $\epsilon \rightarrow 0$, it must be true that $\left(c_{i}, f_{i}\right),(w, v)$ and $\left(c_{j}, f_{j}\right)$ are aligned.

Assume now the other possible case: $i$ bids in $A_{j h}^{i}$ then $j$ bids in $A_{l^{\prime} i}^{j}$. A similar analysis yields that $\left(c_{j}, f_{j}\right),(w, v)$ and $\left(c_{i}, f_{i}\right)$ are aligned. In both cases, we have that $(w, v)$ belongs to the segment $\left[\left(c_{i}, f_{i}\right) ;\left(c_{j}, f_{j}\right)\right]$.

\section{Proposition 3}

Proof. Assume that supplier $i$ is not active in an equilibrium of the game, that its profit is 0 . Define the function $Z^{\left(\mathbf{w}_{-i}, \mathbf{v}_{-i}\right)}(\cdot)$ as in Equation (4), the lower envelope made of all bids except $i$ 's. If $f_{i}<Z^{\left(\mathbf{w}_{-i}, \mathbf{v}_{-i}\right)}\left(c_{i}\right)$, then by bidding $\left(c_{i}+\epsilon, f_{i}+\epsilon\right)$, supplier $i$ achieves some positive profit for $\epsilon$ small enough. This contradicts the previous hypothesis and therefore we must have $f_{i} \geq Z^{\left(\mathbf{w}_{-i}, \mathbf{v}_{-i}\right)}\left(c_{i}\right)$. 
Construct the lower envelope $C^{-i}(\cdot)$ of the costs $\left(c_{1}, f_{1}\right), \ldots,\left(c_{i-1}, f_{i-1}\right),\left(c_{i+1}, f_{i+1}\right), \ldots,\left(c_{N}, f_{N}\right)$, $(p, 0)$. That is, $C^{-i}(\cdot)=Z^{\left(\mathbf{c}_{-i}, \mathbf{f}_{-i}\right)}(\cdot)$. Assume that $C^{-i}(\cdot)$ is not a lower bound on the function $Z^{\left(\mathbf{w}_{-i}, \mathbf{v}_{-i}\right)}(\cdot)$. This implies that there is an active bid $\left(w_{j}, v_{j}\right)$ such that $v_{j}<C^{-i}\left(w_{j}\right) . j \neq i$ since $i$ is not active and is not defining the function $Z^{\left(\mathbf{w}_{-i}, \mathbf{v}_{-i}\right)}(\cdot)$. We claim that if supplier $j$ bids in some region $A_{l h}$, it cannot be at equilibrium. Indeed, we can use Equation (12), in particular,

$$
\begin{gathered}
\frac{d J_{l h}}{d y_{j-}} \geq\left(f_{j}-v_{l}\right)+\left(c_{j}-w_{l}\right) \bar{F}\left(y_{j-}\right), \\
\frac{d J_{l h}}{d y_{j+}} \leq\left(v_{h}-f_{j}\right)+\left(w_{h}-c_{j}\right) \bar{F}\left(y_{j+}\right) .
\end{gathered}
$$

If this is an equilibrium, then $j$ 's bid in $A_{l h}$ must be such that $\left(f_{j}-v_{l}\right)+\left(c_{j}-w_{l}\right) \bar{F}\left(y_{j-}\right) \leq 0$ and $\left(v_{h}-f_{j}\right)+\left(w_{h}-c_{j}\right) \bar{F}\left(y_{j+}\right) \geq 0$. This is equivalent to

$$
f_{j} \leq v_{l}+\left(v_{j}-v_{l}\right) \frac{c_{j}-w_{l}}{w_{j}-w_{l}}
$$

or if $l=0, f_{j}+c_{j} \leq v_{k}+w_{k}$ for some $k$, and

$$
f_{j} \leq v_{h}+\left(v_{j}-v_{h}\right) \frac{w_{h}-c_{j}}{w_{h}-w_{j}} .
$$

But if all this feasible area is not strictly below $C^{-i}(\cdot)$, we can find some other supplier $k$ bidding next to $j$ that also satisfies $v_{k}<C^{-i}\left(w_{k}\right)$. By repeating the argument, we must find a third supplier $l$ satisfying $v_{l}<C^{-i}\left(w_{l}\right)$ that is not $j$ (so no cycling possible). When we reach the supplier with the smallest $w$ or with the biggest $w$ (the dummy supplier, $N+1$ ), we reach a contradiction: for the smallest $w$, we cannot find a different supplier satisfying the condition, for the dummy supplier $v_{N+1}=f_{N+1}=0=C^{-i}\left(c_{N+1}\right)=C^{-i}\left(w_{N+1}\right)=C^{-i}(p)$. Hence $j$ cannot be in equilibrium, and this is a contradiction.

Therefore, the function $C^{-i}(\cdot)$ lies below the function $Z^{\left(\mathbf{w}_{-i}, \mathbf{v}_{-i}\right)}(\cdot)$. This implies that $i$ cannot be efficient, since it is not needed to define the function $C^{-i}(\cdot)$, and thus is not a winning point of $\left\{\left(c_{1}, f_{1}\right), \ldots,\left(c_{N}, f_{N}\right),(p, 0)\right\}$.

\section{Proposition 4}

Proof. Using Proposition 3, we know that every supplier is active in equilibrium.

If the proposition was false, we could find suppliers $i$ and $j$ such that $c_{i}<c_{j}$ and $w_{i}>w_{j}$. We may furthermore assume without loss of generality that these are consecutive bidders, i.e. there is no bid $(w, v)$ with $w_{j}<w<w_{i}$. To see this, assume that the active suppliers are indexed such that $w_{1} \leq \ldots \leq w_{t}$ and in case of a tie, sorted by increasing execution cost $c$.

Select a pair $(i, j)$ such that $i+1<j$ with $w_{i}<w_{j}$ and $c_{i}>c_{j}$. One of the following three cases is possible. 
- The pair $(i, i+1)$ satisfies $w_{i}<w_{i+1}$ and $c_{i}>c_{i+1}$ and then $(i, i+1)$ are consecutive bidders.

- $w_{i}<w_{i+1}$ and $c_{i} \leq c_{i+1}$. Then, it is the pair $(i+1, j)$ that satisfies $c_{i+1}>c_{j}$ and $w_{i+1}<w_{j}$.

Hence, we can iterate this argument until we find consecutive bidders $i$ and $j$ such that $c_{i}<c_{j}$ and $w_{i}>w_{j}$.

- $w_{i}=w_{i+1}$ but then, by construction, $c_{i} \leq c_{i+1}$. Hence, similarly to the previous case, we iterate the argument with the pair $(i+1, j)$.

Since $w_{i}>w_{j}$ and $i$ and $j$ are consecutive bidders, the bid of supplier $j$ must be in the border of some region $A_{l i}$ (where there is no active supplier between $l$ and $i$ because if there was one it would not be active), where supplier $l$ is active. Also, $w_{i}>w_{j}$ implies that $w_{j}=w_{l}$ and $v_{j}=v_{l}$ is optimal, from Theorem 2. But applying Proposition 2 yields that $\left(w_{j}, v_{j}\right)$ belongs in the segment $\left[\left(c_{l}, f_{l}\right) ;\left(c_{j}, f_{j}\right)\right]$. Similarly, supplier $i$ bids in some region $A_{j h}^{\left(\mathbf{w}_{-i}, \mathbf{v}_{-i}\right)}$, with supplier $h$ active and no active suppliers between $j$ and $h$. With the same argument as before, we have that $\left(w_{i}, v_{i}\right)=\left(w_{h}, v_{h}\right)$ and this bid belongs in the segment $\left[\left(c_{i}, f_{i}\right) ;\left(c_{h}, f_{h}\right)\right]$.

Define,

$$
\begin{aligned}
& \bar{F}\left(y_{m}\right)=\frac{v_{j}-v_{i}}{w_{i}-w_{j}}, \\
& \bar{F}\left(y_{j 1}\right)=\frac{v_{j}-f_{j}}{c_{j}-w_{j}}, \\
& \bar{F}\left(y_{i 2}\right)=\frac{v_{i}-f_{i}}{c_{i}-w_{i}},
\end{aligned}
$$

and we have that $y_{j 1}<y_{m}<y_{i 2}$ because $i$ and $j$ are active.

We can also define

$$
\begin{aligned}
& \bar{F}\left(y_{i 1}\right)=\frac{v_{j}-f_{i}}{c_{i}-w_{j}}, \\
& \bar{F}\left(y_{j 2}\right)=\frac{f_{j}-v_{i}}{w_{i}-c_{j}} .
\end{aligned}
$$

Since $c_{i}<c_{j}$, and supplier $i$ is efficient, we must have that $f_{i} \leq f_{j}+\left(c_{j}-c_{i}\right) \bar{F}\left(y_{j 1}\right)$ because $\bar{F}\left(y_{j 1}\right)$ is the slope of the line joining $\left(c_{l}, f_{l}\right)$ to $\left(c_{j}, f_{j}\right)$. Similarly, $f_{j} \leq f_{i}-\left(c_{j}-c_{i}\right) \bar{F}\left(y_{i 2}\right)$. This implies that $y_{i 1} \leq y_{j 1}<y_{m}<y_{i 2} \leq y_{j 2}$ as can be seen from Figure 7 .

Finally, we apply Theorem 2. For this purpose, define the functions

$$
\phi\left(y_{1}\right)=\frac{\int_{y_{1}}^{y_{m}}\left[\bar{F}\left(y_{1}\right)-\bar{F}(u)\right] d u}{\bar{F}\left(y_{1}\right)-\bar{F}\left(y_{m}\right)}
$$

and

$$
\psi\left(y_{2}\right)=\frac{\int_{y_{m}}^{y_{2}}\left[\bar{F}(u)-\bar{F}\left(y_{2}\right)\right] d u}{\bar{F}\left(y_{m}\right)-\bar{F}\left(y_{2}\right)} .
$$




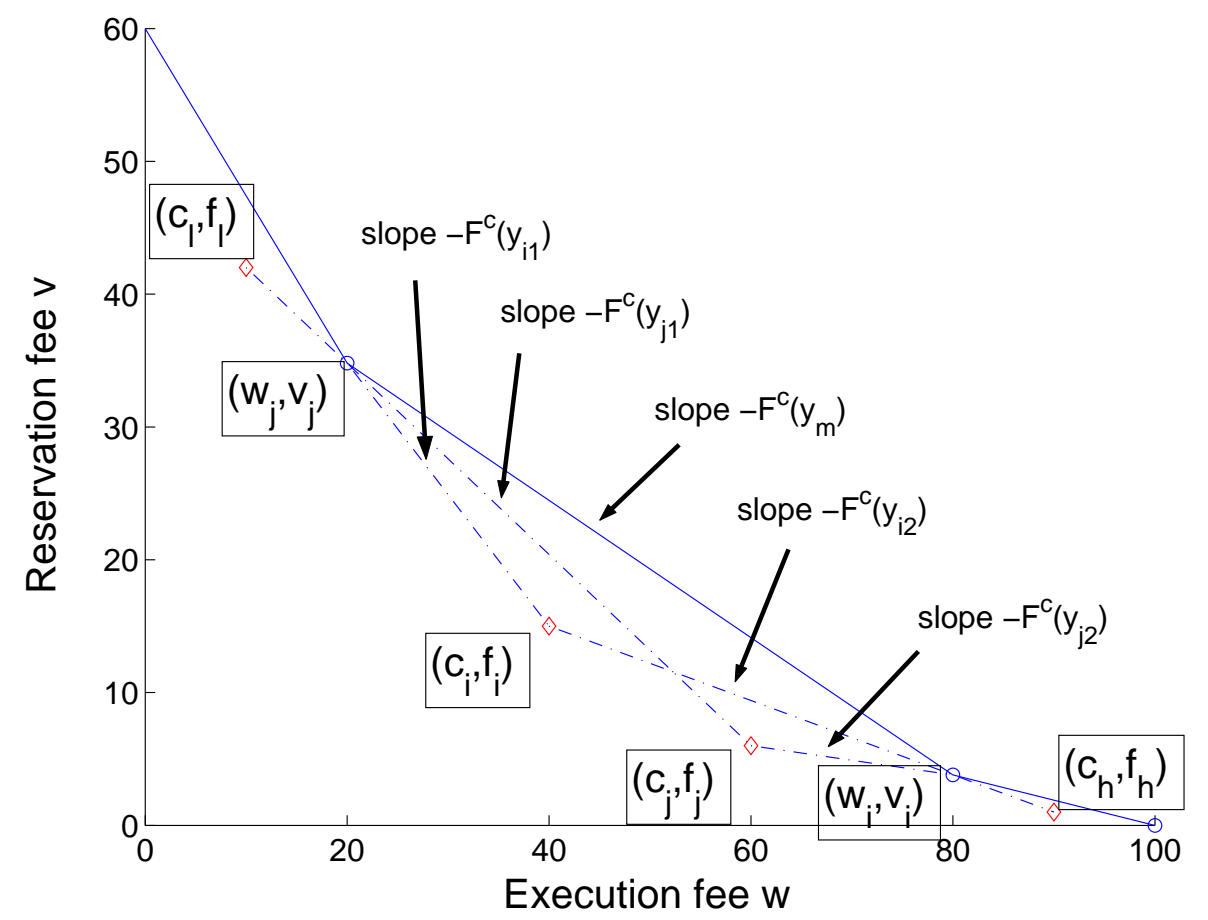

Figure 7: Geometric situation of costs $\left(c_{i}, f_{i}\right)$ and $\left(c_{j}, f_{j}\right)$ in region $A_{l h}$

Taking derivatives, we have, for $y_{1}>y_{m}$ and $y_{2}<y_{m}$,

$$
\begin{aligned}
\phi^{\prime}\left(y_{1}\right) & =-f\left(y_{1}\right) \frac{\int_{y_{1}}^{y_{m}}\left[\bar{F}(u)-\bar{F}\left(y_{m}\right)\right] d u}{\left[\bar{F}\left(y_{1}\right)-\bar{F}\left(y_{m}\right)\right]^{2}}<0 \\
\psi^{\prime}\left(y_{2}\right) & =f\left(y_{2}\right) \frac{\int_{y_{m}}^{y_{2}}\left[\bar{F}\left(y_{m}\right)-\bar{F}(u)\right] d u}{\left[\bar{F}\left(y_{m}\right)-\bar{F}\left(y_{2}\right)\right]^{2}}>0 .
\end{aligned}
$$

Hence, $\phi(\cdot)$ is non-increasing and $\psi(\cdot)$ is non-decreasing.

We now apply the last case of Theorem 2. Since supplier $i$ bids $\left(w_{i}, v_{i}\right)$ and not $\left(w_{j}, v_{j}\right)$, we have $\phi\left(y_{i 1}\right) \leq \psi\left(y_{i 2}\right)$. Similarly, for $j, \phi\left(y_{j 1}\right) \geq \psi\left(y_{j 2}\right) . \quad y_{i 1} \leq y_{j 1}<y_{m}<y_{i 2} \leq y_{j 2}$ yields $\phi\left(y_{i 1}\right) \geq \phi\left(y_{j 1}\right) \geq \psi\left(y_{j 2}\right) \geq \psi\left(y_{i 2}\right)$, and hence $\phi\left(y_{i 1}\right) \leq \psi\left(y_{i 2}\right)$ implies that all inequalities are in fact equalities. Therefore $c_{i}=c_{j}$ which is a contradiction.

\section{Theorem 3}

Proof. Consider supplier $1<i \leq N$. From Propositions 3 and 4 , we know that at equilibrium it will be bidding in region $A_{i-1} i+1$ because otherwise one of the suppliers would be inactive or they would not be sorted in the correct order. Let $l=i-1$ and $h=i+1$. Supplier $i$ will in particular bid in the border of this region, with $y_{i-}=y_{m}$ or $y_{i+}=y_{m}$ as established in Theorem 2. $y_{i-}=y_{m}$ is equivalent to saying that it is bidding $w_{i}=w_{i+1}$ and $v_{i}=v_{i+1}$ and $\bar{F}\left(y_{i+}\right)=\frac{f_{i}-v_{i+1}}{w_{i+1}-c_{i}}$. In 
this case, applying Proposition 2 yields that $\left(v_{i}, w_{i}\right)$ belongs in the segment $\left[\left(c_{i}, f_{i}\right) ;\left(c_{i+1}, f_{i+1}\right)\right]$. Similarly, $y_{i+}=y_{m}$ implies that $\left(v_{i}, w_{i}\right)$ belongs in the segment $\left[\left(c_{i-1}, f_{i-1}\right) ;\left(c_{i}, f_{i}\right)\right]$, and this is of course possible only if $i>1$. For $i=1$, only the first case can occur, i.e., $w_{1}=w_{2}, v_{1}=v_{2}, y_{1-}=0$ and $y_{1+}$ such that $\bar{F}\left(y_{1+}\right)=\frac{f_{1}-v_{2}}{w_{2}-c_{1}}$. Again, Proposition 2 implies that $\left(v_{1}, w_{1}\right)$ belongs in the segment $\left[\left(c_{1}, f_{1}\right) ;\left(c_{2}, f_{2}\right)\right]$.

\section{Theorem 4}

In the following proofs, let, for each demand distribution, for $x \leq y$,

$$
L(x, y)=\frac{\int_{x}^{y}[\bar{F}(x)-\bar{F}(u)] d u}{\bar{F}(x)-\bar{F}(y)},
$$

and

$$
R(x, y)=\frac{\int_{x}^{y}[\bar{F}(u)-\bar{F}(y)] d u}{\bar{F}(x)-\bar{F}(y)}=(y-x)-L(x, y) .
$$

Observe that $L(x, y)$ is non-increasing in $x$ and $R(x, y)$ is non-decreasing in $y$.

Proof. The loss in surplus occurs for every supplier $i$ when $\left(w_{i}, v_{i}\right)=\left(w_{i-1}, v_{i-1}\right)$ and $\left(w_{i}, v_{i}\right) \neq$ $\left(w_{i+1}, v_{i+1}\right)$. For all other cases, we have that $y_{i}=y_{i}^{*}$. We have two different possible cases.

(A) The market allocation is such that $y_{i}<y_{i}^{*}$.

(B) The market allocation is such that $y_{i}>y_{i}^{*}$.

In case $(\mathrm{A})$, Equation $(16)$ holds since $\left(w_{i}, v_{i}\right)=\left(w_{i-1}, v_{i-1}\right)$. Therefore, using $y_{1}=y_{i-1}^{*}, y_{m}=y_{i}$ and $y_{2} \geq y_{i}^{*}$, and the notation of Equations (33) and (34),

$$
L\left(y_{i-1}^{*}, y_{i}\right) \geq R\left(y_{i}, y_{2}\right) .
$$

Since the function $R(x, y)$ is non-decreasing in $y$ and $y_{2} \geq y_{i}^{*}$, and $L(x, y)$ is non-increasing in $x$ and $0 \leq y_{i-1}^{*}$,

$$
R\left(y_{i}, y_{2}\right) \geq R\left(y_{i}, y_{i}^{*}\right) \text { and } L\left(0, y_{i}\right) \geq L\left(y_{i-1}^{*}, y_{i}\right) .
$$

Thus,

$$
L\left(0, y_{i}\right) \geq R\left(y_{i}, y_{i}^{*}\right)
$$

Examine now the loss created by supplier $i$.

$$
\begin{aligned}
\int_{0}^{y_{i}^{*}}\left[\bar{F}(u)-\bar{F}\left(y_{i}^{*}\right)\right] d u & =\int_{0}^{y_{i}}\left[\bar{F}(u)-\bar{F}\left(y_{i}^{*}\right)\right] d u+\int_{y_{i}}^{y_{i}^{*}}\left[\bar{F}(u)-\bar{F}\left(y_{i}^{*}\right)\right] d u \\
& \geq\left(\bar{F}\left(y_{i}\right)-\bar{F}\left(y_{i}^{*}\right)\right) y_{i}+\left(\bar{F}\left(y_{i}\right)-\bar{F}\left(y_{i}^{*}\right)\right) R\left(y_{i}, y_{i}^{*}\right) \\
& \geq\left(\bar{F}\left(y_{i}\right)-\bar{F}\left(y_{i}^{*}\right)\right)\left(L\left(0, y_{i}\right)+R\left(y_{i}, y_{i}^{*}\right)\right) \\
& \geq\left(\bar{F}\left(y_{i}\right)-\bar{F}\left(y_{i}^{*}\right)\right) 2 R\left(y_{i}, y_{i}^{*}\right), \\
& =2 \int_{y_{i}}^{y_{i}^{*}}\left[\bar{F}(u)-\bar{F}\left(y_{i}^{*}\right)\right] d u .
\end{aligned}
$$


Hence, we have that

$$
\Delta c_{i} \int_{y_{i}}^{y_{i}^{*}}\left[\bar{F}(u)-\bar{F}\left(y_{i}^{*}\right)\right] d u \leq \frac{1}{2} \Delta c_{i} \int_{0}^{y_{i}^{*}}\left[\bar{F}(u)-\bar{F}\left(y_{i}^{*}\right)\right] d u .
$$

In case $(\mathrm{B})$, it must be that $i<N$. Since $\left(w_{i}, v_{i}\right) \neq\left(w_{i+1}, v_{i+1}\right)$, Theorem 3 implies that $\left(w_{i+1}, v_{i+1}\right)=\left(w_{i+2}, v_{i+2}\right)$, and this means that $w_{i} \leq c_{i} \leq c_{i+1} \leq w_{i+1} \leq c_{i+2}, y_{i+1}=y_{i+1}^{*}$ and $y_{i} \leq y_{i+1}^{*}$. We can now use Equation (17) for supplier $i+1$ in order to derive a bound on the loss. Here, $y_{m}=y_{i}, y_{2}=y_{i+1}^{*}$ and $y_{1} \leq y_{i}^{*}$,

$$
R\left(y_{i}, y_{i+1}^{*}\right) \geq L\left(y_{1}, y_{i}\right)
$$

This implies that

$$
R\left(y_{i}, y_{i+1}^{*}\right) \geq L\left(y_{i}^{*}, y_{i}\right)
$$

Now, note that

$$
\begin{aligned}
\left(w_{i+1}-w_{i}\right) \bar{F}\left(y_{i}\right) & =\left(c_{i+1}-w_{i}\right) \bar{F}\left(y_{1}\right)+\left(w_{i+1}-c_{i+1}\right) \bar{F}\left(y_{i+1}^{*}\right) \\
& \geq\left(c_{i+1}-w_{i}\right) \bar{F}\left(y_{i}^{*}\right)+\left(w_{i+1}-c_{i+1}\right) \bar{F}\left(y_{i+1}^{*}\right),
\end{aligned}
$$

where the inequality is justified by $c_{i+1} \geq w_{i}$ and $y_{1} \leq y_{i}^{*}$. This, together with $\Delta c_{i} \leq c_{i+1}-w_{i}$ and $w_{i+1}-c_{i+1} \leq \Delta c_{i+1}$, implies that

$$
\begin{aligned}
\Delta c_{i}\left[\bar{F}\left(y_{i}^{*}\right)-\bar{F}\left(y_{i}\right)\right] & \leq\left(c_{i+1}-w_{i}\right)\left[\bar{F}\left(y_{i}^{*}\right)-\bar{F}\left(y_{i}\right)\right] \\
& \leq\left(w_{i+1}-c_{i+1}\right)\left[\bar{F}\left(y_{i}\right)-\bar{F}\left(y_{i+1}^{*}\right)\right] \\
& \leq \Delta c_{i+1}\left[\bar{F}\left(y_{i}\right)-\bar{F}\left(y_{i+1}^{*}\right)\right] .
\end{aligned}
$$

Since

$$
\begin{aligned}
\int_{0}^{y_{i+1}^{*}}\left[\bar{F}(u)-\bar{F}\left(y_{i+1}^{*}\right)\right] d u & =\int_{0}^{y_{i}}\left[\bar{F}(u)-\bar{F}\left(y_{i+1}^{*}\right)\right] d u+\int_{y_{i}}^{y_{i+1}^{*}}\left[\bar{F}(u)-\bar{F}\left(y_{i+1}^{*}\right)\right] d u \\
& \geq\left(\bar{F}\left(y_{i}\right)-\bar{F}\left(y_{i+1}^{*}\right)\right)\left(L\left(y_{i}^{*}, y_{i}\right)+R\left(y_{i}, y_{i+1}^{*}\right)\right) \\
& \geq\left(\bar{F}\left(y_{i}\right)-\bar{F}\left(y_{i+1}^{*}\right)\right) 2 L\left(y_{i}^{*}, y_{i}\right),
\end{aligned}
$$

we have that

$$
\Delta c_{i+1} \int_{0}^{y_{i+1}^{*}}\left[\bar{F}(u)-\bar{F}\left(y_{i+1}^{*}\right)\right] d u \geq \Delta c_{i}\left(\bar{F}\left(y_{i}^{*}\right)-\bar{F}\left(y_{i}\right)\right) 2 L\left(y_{i}^{*}, y_{i}\right)
$$

and hence

$$
\Delta c_{i} \int_{y_{i}}^{y_{i}^{*}}\left[\bar{F}(u)-\bar{F}\left(y_{i}^{*}\right)\right] d u \leq \frac{1}{2} \Delta c_{i+1} \int_{0}^{y_{i+1}^{*}}\left[\bar{F}(u)-\bar{F}\left(y_{i+1}^{*}\right)\right] d u .
$$

Since $y_{i+1}=y_{i+1}^{*}$, this completes the proof of the bound for any border distribution. 


\section{Proof of Theorem 5}

Let $\mathcal{F}$ be the set of log-concave distributions. We present two lemmas which, combined, provide the proof of the theorem.

Lemma 1 When all suppliers are efficient, then in every equilibrium of the game

$$
\Delta U \leq 25 \% U^{*}
$$

provided that for each $0 \leq x \leq y \leq z$, such that $L(x, y) \geq R(y, z)$, where the functions $L$ and $R$ are defined in Equations (33) and (34) respectively,

$$
[\bar{F}(y)-\bar{F}(z)][2 L(x, y)-R(x, y)]-[\bar{F}(x)-\bar{F}(y)] R(x, y) \leq 0
$$

Proof. Assume that the condition defined in Equation (35) is satisfied for all $0 \leq x \leq y \leq z$, such that $L(x, y) \geq R(y, z)$.

The loss in surplus $U$ occurs when $y_{i} \neq y_{i}^{*}$. That happens when a supplier $i$ bids $\left(w_{i}, v_{i}\right)=$ $\left(w_{i-1}, v_{i-1}\right)$ and $\left(w_{i}, v_{i}\right) \neq\left(w_{i+1}, v_{i+1}\right)$. When $\left(w_{i}, v_{i}\right) \neq\left(w_{i-1}, v_{i-1}\right)$ and $\left(w_{i}, v_{i}\right)=\left(w_{i+1}, v_{i+1}\right)$, $y_{i}=y_{i}^{*}$.

For the situation when loss is created, since bidding with supplier $i-1$ yields the maximum profit for supplier $i$, then by Theorem 2 , we have that

$$
L\left(y_{i-1}^{*}, y_{i}\right) \geq R\left(y_{i}, y_{2}\right)
$$

where $y_{2}$ is defined by $\bar{F}\left(y_{2}\right)=\frac{f_{i}-v_{i+1}}{w_{i+1}-c_{i}}$. We have two different possible cases.

(A) The market allocation is such that $y_{i}<y_{i}^{*}$.

(B) The market allocation is such that $y_{i}>y_{i}^{*}$.

In case $(\mathrm{A})$, since $y_{2} \geq y_{i}^{*}$ and $y_{i-1}^{*} \geq 0$, Equation (36) yields that $L\left(0, y_{i}\right) \geq R\left(y_{i}, y_{i}^{*}\right)$.

We claim that in this case $(\mathrm{A})$, we have

$$
\int_{y_{i}}^{y_{i}^{*}}\left[\bar{F}(u)-\bar{F}\left(y_{i}^{*}\right)\right] d u \leq \frac{1}{4} \int_{0}^{y_{i}^{*}}\left[\bar{F}(u)-\bar{F}\left(y_{i}^{*}\right)\right] d u .
$$

This is equivalent to saying that

$$
\left[\bar{F}\left(y_{i}\right)-\bar{F}\left(y_{i}^{*}\right)\right] R\left(y_{i}, y_{i}^{*}\right) \leq \frac{1}{4}\left\{\begin{array}{l}
{\left[1-\bar{F}\left(y_{i}\right)\right] R\left(0, y_{i}\right)} \\
+\left[\bar{F}\left(y_{i}\right)-\bar{F}\left(y_{i}^{*}\right)\right]\left[R\left(0, y_{i}\right)+L\left(0, y_{i}\right)\right] \\
\left.+\bar{F}\left(y_{i}\right)-\bar{F}\left(y_{i}^{*}\right)\right] R\left(y_{i}, y_{i}^{*}\right)
\end{array}\right\}
$$

or put differently,

$$
\left[\bar{F}\left(y_{i}\right)-\bar{F}\left(y_{i}^{*}\right)\right]\left[3 R\left(y_{i}, y_{i}^{*}\right)-R\left(0, y_{i}\right)-L\left(0, y_{i}\right)\right] \leq\left[1-\bar{F}\left(y_{i}\right)\right] R\left(0, y_{i}\right) .
$$


Since $R\left(y_{i}, y_{i}^{*}\right) \leq L\left(0, y_{i}\right)$, to prove Equation (37), it is sufficient to show that

$$
\left[\bar{F}\left(y_{i}\right)-\bar{F}\left(y_{i}^{*}\right)\right]\left[2 L\left(0, y_{i}\right)-R\left(0, y_{i}\right)\right] \leq\left[1-\bar{F}\left(y_{i}\right)\right] R\left(0, y_{i}\right),
$$

which is exactly the condition that we assumed true. This concludes the proof of case (A), i.e.,

$$
\int_{y_{i}}^{y_{i}^{*}}\left[\bar{F}(u)-\bar{F}\left(y_{i}^{*}\right)\right] d u \leq \frac{1}{4} \int_{0}^{y_{i}^{*}}\left[\bar{F}(u)-\bar{F}\left(y_{i}^{*}\right)\right] d u .
$$

In case $(\mathrm{B})$, it must be that $i<N$. Since $\left(w_{i}, v_{i}\right) \neq\left(w_{i+1}, v_{i+1}\right)$, Theorem 3 implies that $\left(w_{i+1}, v_{i+1}\right)=\left(w_{i+2}, v_{i+2}\right)$, and this means that $w_{i} \leq c_{i} \leq c_{i+1} \leq w_{i+1} \leq c_{i+2}, y_{i+1}=y_{i+1}^{*}$ and $y_{i} \leq y_{i+1}^{*}$. We can now use Theorem 2, for supplier $i+1$, to yield

$$
R\left(y_{i}, y_{i+1}^{*}\right) \geq L\left(y_{1}, y_{i}\right) \geq L\left(y_{i}^{*}, y_{i}\right),
$$

where $y_{1}$ is defined by $\bar{F}\left(y_{1}\right)=\frac{v_{i}-f_{i+1}}{c_{i+1}-w_{i}}$, which implies that $y_{1} \leq y_{i}^{*}$.

We claim that when $R\left(y_{i}, y_{i+1}^{*}\right) \geq L\left(y_{i}^{*}, y_{i}\right)$, then

$$
\Delta c_{i} \int_{y_{i}^{*}}^{y_{i}}\left[\bar{F}\left(y_{i}^{*}\right)-\bar{F}(u)\right] d u \leq \frac{1}{4} \Delta c_{i+1} \int_{y_{i}^{*}}^{y_{i+1}^{*}}\left[\bar{F}(u)-\bar{F}\left(y_{i+1}^{*}\right)\right] d u .
$$

Since the right-hand side is non-decreasing in $y_{i+1}^{*}$, it is sufficient to show that when $R\left(y_{i}, y_{i+1}^{*}\right)=$ $L\left(y_{i}^{*}, y_{i}\right)$, Equation (38) is satisfied.

We must first note that

$$
\begin{aligned}
\left(w_{i+1}-w_{i}\right) \bar{F}\left(y_{i}\right) & =\left(c_{i+1}-w_{i}\right) \bar{F}\left(y_{1}\right)+\left(w_{i+1}-c_{i+1}\right) \bar{F}\left(y_{i+1}^{*}\right) \\
& \geq\left(c_{i+1}-w_{i}\right) \bar{F}\left(y_{i}^{*}\right)+\left(w_{i+1}-c_{i+1}\right) \bar{F}\left(y_{i+1}^{*}\right),
\end{aligned}
$$

where the inequality is justified by $c_{i+1} \geq w_{i}$ and $y_{1} \leq y_{i}^{*}$. This, together with $\Delta c_{i} \leq c_{i+1}-w_{i}$ and $w_{i+1}-c_{i+1} \leq \Delta c_{i+1}$, implies that

$$
\begin{aligned}
\Delta c_{i}\left[\bar{F}\left(y_{i}^{*}\right)-\bar{F}\left(y_{i}\right)\right] & \leq\left(c_{i+1}-w_{i}\right)\left[\bar{F}\left(y_{i}^{*}\right)-\bar{F}\left(y_{i}\right)\right] \\
& \leq\left(w_{i+1}-c_{i+1}\right)\left[\bar{F}\left(y_{i}\right)-\bar{F}\left(y_{i+1}^{*}\right)\right] \\
& \leq \Delta c_{i+1}\left[\bar{F}\left(y_{i}\right)-\bar{F}\left(y_{i+1}^{*}\right)\right] .
\end{aligned}
$$

Thus, in order to prove Equation (38), it is sufficient to show that

$$
\frac{\int_{y_{i}^{*}}^{y_{i}}\left[\bar{F}\left(y_{i}^{*}\right)-\bar{F}(u)\right] d u}{\left[\bar{F}\left(y_{i}^{*}\right)-\bar{F}\left(y_{i}\right)\right]} \leq \frac{1}{4} \frac{\int_{y_{i}^{*}}^{y_{i+1}^{*}}\left[\bar{F}(u)-\bar{F}\left(y_{i+1}^{*}\right)\right] d u}{\bar{F}\left(y_{i}\right)-\bar{F}\left(y_{i+1}^{*}\right)},
$$

or equivalently,

$$
\left[\bar{F}\left(y_{i}\right)-\bar{F}\left(y_{i+1}^{*}\right)\right] L\left(y_{i}^{*}, y_{i}\right) \leq \frac{1}{4}\left\{\begin{array}{l}
{\left[\bar{F}\left(y_{i}\right)-\bar{F}\left(y_{i+1}^{*}\right)\right] R\left(y_{i}, y_{i+1}^{*}\right)} \\
+\left[\bar{F}\left(y_{i}\right)-\bar{F}\left(y_{i+1}^{*}\right)\right]\left[L\left(y_{i}^{*}, y_{i}\right)+R\left(y_{i}^{*}, y_{i}\right)\right] \\
\left.+\bar{F}\left(y_{i}^{*}\right)-\bar{F}\left(y_{i}\right)\right] R\left(y_{i}^{*}, y_{i}\right)
\end{array}\right\} .
$$


Using that $L\left(y_{i}^{*}, y_{i}\right)=R\left(y_{i}, y_{i+1}^{*}\right)$, it is sufficient to show that

$$
\left[\bar{F}\left(y_{i}\right)-\bar{F}\left(y_{i+1}^{*}\right)\right]\left[2 L\left(y_{i}^{*}, y_{i}\right)-R\left(y_{i}^{*}, y_{i}\right)\right] \leq\left[\bar{F}\left(y_{i}^{*}\right)-\bar{F}\left(y_{i}\right)\right] R\left(y_{i}^{*}, y_{i}\right) .
$$

Again, using the condition defined in Equation (35), this is non-positive. This implies that in case (B), for all $y_{i}^{*}, y_{i}, y_{i+1}^{*}$ such that $R\left(y_{i}, y_{i+1}^{*}\right) \geq L\left(y_{i}^{*}, y_{i}\right)$,

$$
\Delta c_{i} \int_{y_{i}}^{y_{i}^{*}}\left[\bar{F}(u)-\bar{F}\left(y_{i}^{*}\right)\right] d u \leq \frac{1}{4} \Delta c_{i+1} \int_{0}^{y_{i+1}^{*}}\left[\bar{F}(u)-\bar{F}\left(y_{i+1}^{*}\right)\right] d u .
$$

Finally, putting together cases (A) and (B), we have

$$
\begin{aligned}
\Delta U & =\sum_{i=1}^{N} \Delta c_{i} \int_{y_{i}}^{y_{i}^{*}}\left[\bar{F}(u)-\bar{F}\left(y_{i}^{*}\right)\right] d u \leq \frac{1}{4} \sum_{i=1}^{N} \Delta c_{i} \int_{0}^{y_{i}^{*}}\left[\bar{F}(u)-\bar{F}\left(y_{i}^{*}\right)\right] d u \\
& \leq \frac{1}{4} U^{*} .
\end{aligned}
$$

Lemma 2 When $f$ is log-concave, then for each $0 \leq x \leq y \leq z$, such that $L(x, y) \geq R(y, z)$,

$$
[\bar{F}(y)-\bar{F}(z)][2 L(x, y)-R(x, y)]-[\bar{F}(x)-\bar{F}(y)] R(x, y) \leq 0 .
$$

Proof. Let's examine the worst-case scenario. For a fixed $x$, we claim that

$$
\sup _{x \leq y \leq z, F \in \mathcal{F}}\left\{\begin{array}{c}
{[\bar{F}(y)-\bar{F}(z)][2 L(x, y)-R(x, y)]} \\
-[\bar{F}(x)-\bar{F}(y)] R(x, y)
\end{array}\right\} \leq 0
$$

Clearly, we only need to examine the case when $2 L(x, y) \geq R(x, y)$. The objective is then maximized for the largest $z$ feasible, given $y$ and $F$. This implies that at the maximum, $L(x, y)=$ $R(y, z)$, since $R(y, z)$ is non-decreasing in $z$.

Let's now examine the worst-case scenario in terms of distribution. We first need to define the following subclass of log-concave distributions.

Definition $4 A$ distribution is truncated exponential on $I \subset \mathbb{R}_{+}$if and only if there are $\beta, K, a, b$, $a<b$, such that for $t \in I$,

$$
f(t)=K e^{\beta t} \mathbf{1}_{[a, b]}(t) .
$$

Without loss of generality, we can assume that $x=0$, since for any other $x \geq 0$ we could prove the lemma with a shifted distribution.

Claim 1 In the problem posed by Equation (39), given optimal $x=0, y, z$, we claim that at the optimum $f$ must be truncated exponential in $[y, z]$, with a rate equal to $f^{\prime}(y) / f(y)$. 
Proof. We have two possible cases. Either $\bar{F}(z)=0$ or not. If $\bar{F}(z)=0, \bar{F}(y)>0$, otherwise there is nothing to show. Assume that $f$ is not truncated exponential. Define the distribution equal to $f$ on $[0, y]$ and to the truncated exponential

$$
g_{\gamma}(t)=f(y) e^{f^{\prime}(y) / f(y)(t-y)} \mathbf{1}[y, \gamma]
$$

on $[y, \infty)$. Since $f$ is log-concave, then $f(t) \leq g_{\gamma}(t)$ for $y \leq t \leq \gamma$.

Define $\overline{G_{\gamma}}$ such that

$$
\overline{G_{\gamma}}(t)=\bar{F}(y)-\int_{y}^{t} g_{\gamma}(u) d u=\int_{y}^{z} f(u) d u-\int_{y}^{t} g_{\gamma}(u) d u
$$

This is clearly increasing in $\gamma$. We have $\overline{G_{y}}(z)>0$ and $\overline{G_{z}}(z)<0$. We can thus find $\gamma$ such that $\overline{G_{\gamma}}(z)=0$, and hence for this particular $\gamma$,

$$
\bar{F}(y)-\bar{F}(z)=\int_{y}^{z} g_{\gamma}(u) d u
$$

Moreover, $L(0, y)=R(y, z)<\frac{\int_{y}^{z}\left[\overline{G_{\gamma}}(u)-\overline{G_{\gamma}}(z)\right] d u}{\overline{G_{\gamma}}(y)-\overline{G_{\gamma}}(z)}$. This implies that for the log-concave distribution $g_{\gamma}$, we can decrease $z$ to $z^{\prime}$ with $g_{\gamma}\left(z^{\prime}\right)>0$, while still satisfying the feasibility constraint, thus increasing $\bar{F}(y)-\bar{F}(z)$ to a larger quantity $\overline{G_{\gamma}}(y)-\overline{G_{\gamma}}\left(z^{\prime}\right)$. Thus $f$ cannot be the worst-case distribution. The only remaining possibility is that $f$ is truncated exponential on $[y, \infty)$, with rate $f^{\prime}(y) / f(y)$.

Finally, if $\bar{F}(z)>0, f(z)>0$ and $\bar{F}(y)>0$. Assume that $f$ is not exponential. Define, for $f^{\prime}(y) / f(y) \geq \gamma \geq f^{\prime}(z) / f(z)$, the distribution equal to $f$ on $[0, y]$ and $[z, \infty)$, and to

$$
g_{\gamma}(t)=\min \left\{f(y) e^{f^{\prime}(y) / f(y)(t-y)}, f(z) e^{\gamma(t-z)}\right\}
$$

on $[y, z]$. This is clearly log-concave. Fix $\gamma$ such that

$$
\bar{F}(y)-\bar{F}(z)=\int_{y}^{z} g_{\gamma}(u) d u
$$

This implies that

$$
\overline{G_{\gamma}}(t)=\bar{F}(z)+\int_{t}^{z} g_{\gamma}(u) d u
$$

is always greater than $\bar{F}(t)$. Thus $L(0, y)=R(y, z) \leq \frac{\int_{y}^{z}\left[\overline{G_{\gamma}}(u)-\overline{G_{\gamma}}(z)\right] d u}{\overline{G_{\gamma}}(y)-\overline{G_{\gamma}}(z)}$. Hence, for the $\log$-concave distribution $g_{\gamma}$, we can decrease $z$ while still satisfying the feasibility constraint, thus increasing $\bar{F}(y)-\bar{F}(z)$ to a larger quantity. Thus $f$ cannot be the worst-case distribution. The only possibility is that $f$ is exponential, with rate $f^{\prime}(y) / f(y)$.

In any case, we have showed that for the worst case distribution must be truncated exponential in $[y, z]$. 
Claim 2 In the problem posed by Equation (39), given optimal x,y, z, we claim that at the optimum $f$ must be truncated exponential in $[x, y]$.

Proof. Equation (39) can be rewritten as

$$
\sup _{x \leq y \leq z, F \in \mathcal{F}}\left\{\begin{array}{c}
{[\bar{F}(y)-\bar{F}(z)] 2 R(y, z)} \\
-[\bar{F}(x)-\bar{F}(z)] R(x, y)
\end{array}\right\} \leq 0
$$

The proof is similar to the proof of the previous claim.

We have two cases to address: either $f(x)=0$ or not. When $f(x)=0$, assume that $f$ is not truncated exponential. Define the distribution equal to $f$ on $[y, \infty)$ and to the truncated exponential

$$
g_{\gamma}(t)=f(y) e^{f^{\prime}(y) / f(y)(t-y)} \mathbf{1}[\gamma, y]
$$

on $[0, y]$. Since $f$ is log-concave, then $f(t) \leq g_{\gamma}(t)$ for $\gamma \leq t \leq y$.

Define $\overline{G_{\gamma}}$ such that

$$
\overline{G_{\gamma}}(t)=\bar{F}(y)+\int_{t}^{y} g_{\gamma}(u) d u .
$$

This is clearly decreasing in $\gamma$. We have $\overline{G_{y}}(x)<1$ and $\overline{G_{x}}(x)>1$. We can thus find $\gamma$ such that $\overline{G_{\gamma}}(x)=1$, and hence for this particular $\gamma$,

$$
1-\bar{F}(y)=\bar{F}(x)-\bar{F}(y)=\int_{x}^{y} g_{\gamma}(u) d u .
$$

Moreover, $R(y, z)=L(x, y)>\frac{\int_{x}^{y}\left[\overline{G_{\gamma}}(x)-\overline{G_{\gamma}}(u)\right] d u}{\overline{G_{\gamma}}(x)-\overline{G_{\gamma}}(y)}$. This implies that for the log-concave distribution $g_{\gamma}$, we can increase $x$ to $x^{\prime}$ with $g_{\gamma}\left(x^{\prime}\right)>0$, while still satisfying the feasibility constraint, thus decreasing $[\bar{F}(x)-\bar{F}(z)] R(x, y)$ to a smaller quantity. This is true because $\bar{F}(x)=1$ goes down to $\overline{G_{\gamma}}\left(x^{\prime}\right)$ and $R(x, y)=(y-x)-L(x, y)=(y-x)-R(y, z)$ goes down as well. Thus $f$ cannot be the worst-case distribution. The only remaining possibility is that $f$ is truncated exponential on $[x, y]$.

The last case to consider is that $f(x)>0$. Assume that $f$ is not exponential. Define, for $f^{\prime}(x) / f(x) \geq \gamma \geq f^{\prime}(y) / f(y)$, the distribution equal to $f$ on $[0, x]$ and $[y, \infty)$, and to

$$
g_{\gamma}(t)=\min \left\{f(y) e^{f^{\prime}(y) / f(y)(t-y)}, f(x) e^{\gamma(t-x)}\right\}
$$

on $[x, y]$. This is clearly log-concave. Fix $\gamma$ such that

This implies that

$$
\bar{F}(y)-\bar{F}(z)=\int_{x}^{y} g_{\gamma}(u) d u
$$

$$
\overline{G_{\gamma}}(t)=\bar{F}(y)+\int_{t}^{y} g_{\gamma}(u) d u
$$


is always greater than $\bar{F}(t)$. Thus $R(y, z)=L(x, y)>\frac{\int_{x}^{y}\left[\overline{G_{\gamma}}(x)-\overline{G_{\gamma}}(u)\right] d u}{\overline{G_{\gamma}}(x)-\overline{G_{\gamma}}(y)}$. Hence, for the log-concave distribution $g_{\gamma}$, we can increase $x$ while still satisfying the feasibility constraint, thus decreasing $[\bar{F}(x)-\bar{F}(z)] R(x, y)$ to a smaller quantity. Thus $f$ cannot be the worst-case distribution. The only possibility is that $f$ is exponential.

In any case, we have showed that for the worst case distribution must be truncated exponential in $[x, y]$.

Having proved these two claims, we are ready to complete the proof. The worst-case is obtained for a truncated exponential distribution. We have three different cases to address:

(i) The rate is negative, i.e. $f(t)=K e^{-\beta t} \mathbf{1}_{[a, b]}(t)$ for some parameters $K, a, b, \beta$ with $a<b$ and $\beta>0$.

(ii) The rate is positive, i.e. $f(t)=K e^{\beta t} \mathbf{1}_{[a, b]}(t)$ for some parameters $K, a, b, \beta$ with $a<b$ and $\beta>0$.

(iii) The rate is zero, in which case the distribution is uniform.

We will start with the analysis of case (i). Hence, assume that $f(t)=K e^{-\beta t} \mathbf{1}_{[a, b]}(t)$ for $a<b$ and $\beta>0$. It is clear that for $x \leq a<y<b, L(x, y)=L(a, y)$ and that for $a<x<b \leq y$, $R(x, y)=R(x, b)$. Thus, we can without loss of generality consider the case where $a=0 \leq y_{i} \leq$ $y_{i}^{*} \leq b$.

For this distribution, for all $a \leq x \leq y \leq b$,

$$
L(x, y)=\frac{(y-x) e^{-\beta x}}{e^{-\beta x}-e^{-\beta y}}-\frac{1}{\beta},
$$

and

$$
R(x, y)=\frac{1}{\beta}-\frac{(y-x) e^{-\beta y}}{e^{-\beta x}-e^{-\beta y}} .
$$

Define the following functions

$$
P_{1}(t)=\frac{e^{t}-1}{t} \text { and } P_{2}(t)=\frac{e^{t}-1-t}{t^{2}} .
$$

It is easy to show that these are analytical functions on $\mathbb{R}$, infinitely differentiable, increasing and convex. Using this notation, we can express

$$
L(x, y)=\frac{1}{\beta}\left[\frac{1}{P_{1}(-\beta(y-x))}-1\right],
$$

and

$$
R(x, y)=\frac{1}{\beta}\left[1-\frac{1}{P_{1}(\beta(y-x))}\right],
$$

By writing $\Delta_{1}=\beta(y-x)$ and $\Delta_{2}=\beta(z-y)$, the constraint $L(x, y)=R(y, z)$ thus becomes

$$
\frac{1}{P_{1}\left(-\Delta_{1}\right)}+\frac{1}{P_{1}\left(\Delta_{2}\right)}=2
$$


On the other hand, the objective becomes

$$
\begin{aligned}
& {[\bar{F}(y)-\bar{F}(z)] 2 R(y, z)-[\bar{F}(x)-\bar{F}(z)] R(x, y) } \\
= & \frac{e^{-\beta y}}{\beta^{2}}\left[\begin{array}{l}
\left(1-e^{-\Delta_{2}}\right)\left(\frac{2}{P_{1}\left(-\Delta_{1}\right)}+\frac{1}{P_{1}\left(\Delta_{1}\right)}-3\right) \\
-\left(e^{\Delta_{1}}-1\right)\left(1-\frac{1}{P_{1}\left(\Delta_{1}\right)}\right)
\end{array}\right]
\end{aligned}
$$

We need to show that for $\Delta_{1}, \Delta_{2} \geq 0$ satisfying the constraint, we have

$$
\frac{\left(1-e^{-\Delta_{2}}\right)}{\left(e^{\Delta_{1}}-1\right)}\left(\frac{2}{P_{1}\left(-\Delta_{1}\right)}+\frac{1}{P_{1}\left(\Delta_{1}\right)}-3\right)-\left(1-\frac{1}{P_{1}\left(\Delta_{1}\right)}\right) \leq 0 .
$$

Notice first that since $1 / P_{1}$ is convex, we have that $\Delta_{2} \geq \Delta_{1}$. Note also that

$$
1-\frac{1}{P_{1}\left(\Delta_{2}\right)}=\frac{\Delta_{2} P_{2}\left(\Delta_{2}\right)}{P_{1}\left(\Delta_{2}\right)}=\frac{\left(1-e^{-\Delta_{2}}\right) P_{2}\left(\Delta_{2}\right)}{P_{1}\left(-\Delta_{2}\right) P_{1}\left(\Delta_{2}\right)}
$$

and

$$
\frac{1}{P_{1}\left(-\Delta_{1}\right)}-1=\frac{\Delta_{1} P_{2}\left(-\Delta_{1}\right)}{P_{1}\left(-\Delta_{1}\right)}=\frac{\left(e^{\Delta_{1}}-1\right) P_{2}\left(-\Delta_{1}\right)}{P_{1}\left(-\Delta_{1}\right) P_{1}\left(\Delta_{1}\right)}
$$

Finally, we remark that for all $t$,

$$
\frac{P_{2}(t)}{P_{1}(-t) P_{1}(t)}=\frac{P_{2}(t)}{P_{2}(-t)+P_{2}(t)}
$$

which is an increasing function, because $P_{2}$ is increasing.

The constraint on $\Delta_{1}, \Delta_{2}$, together with $\Delta_{2} \geq \Delta_{1}$, thus implies that

$$
\frac{\left(1-e^{-\Delta_{2}}\right) P_{2}\left(\Delta_{1}\right)}{P_{1}\left(-\Delta_{1}\right) P_{1}\left(\Delta_{1}\right)} \leq \frac{\left(1-e^{-\Delta_{2}}\right) P_{2}\left(\Delta_{2}\right)}{P_{1}\left(-\Delta_{2}\right) P_{1}\left(\Delta_{2}\right)}=\frac{\left(e^{\Delta_{1}}-1\right) P_{2}\left(-\Delta_{1}\right)}{P_{1}\left(-\Delta_{1}\right) P_{1}\left(\Delta_{1}\right)} .
$$

Thus $\left(1-e^{-\Delta_{2}}\right) /\left(e^{\Delta_{1}}-1\right) \leq P_{2}\left(-\Delta_{1}\right) / P_{2}\left(\Delta_{1}\right)$. Hence it is sufficient to show that for all $t \geq 0$,

$$
\frac{P_{2}(-t)}{P_{2}(t)}\left(\frac{2}{P_{1}(-t)}+\frac{1}{P_{1}(t)}-3\right) \leq 1-\frac{1}{P_{1}(t)},
$$

or equivalently, using that $P_{1}(-t)=e^{-t} P_{1}(t), e^{t}=1+t P_{1}(t)$ and $P_{1}(t)=1+t P_{2}(t)$,

$$
t^{4} P_{2}(-t)\left(2 P_{1}(t)-3 P_{2}(t)\right) \leq t^{4} P_{2}(t)^{2} .
$$

Since

$$
\begin{gathered}
t^{4} P_{2}(t)^{2}=e^{2 t}-2 e^{t}-2 t e^{t}+1+2 t+t^{2}=\sum_{k=4}^{\infty} \frac{t^{k}}{k !}\left[2^{k}-2-2 k\right], \\
t^{4} P_{2}(-t) P_{1}(t)=t\left(-e^{t}-e^{-t}+t e^{t}+2-t\right)=\sum_{k=4}^{\infty} \frac{t^{k}}{k !}\left[k(k-1)-k\left(1-(-1)^{k}\right)\right], \\
t^{4} P_{2}(-t) P_{2}(t)=-e^{t}-e^{-t}+t e^{t}-t e^{-t}+2-t^{2}=\sum_{k=4}^{\infty} \frac{t^{k}}{k !}\left[(k-1)\left(1+(-1)^{k}\right)\right],
\end{gathered}
$$


we have,

$$
\begin{aligned}
& -t^{4} P_{2}(-t)\left(2 P_{1}(t)-3 P_{2}(t)\right)+t^{4} P_{2}(t)^{2} \\
= & \sum_{k=4}^{\infty} \frac{t^{k}}{k !}\left[2^{k}-2-2 k+3(k-1)\left(1+(-1)^{k}\right)-2 k(k-1)+2 k\left(1-(-1)^{k}\right)\right] .
\end{aligned}
$$

The term under brackets is always non-negative for $k \geq 4$. Indeed, $1+(-1)^{k} \geq 0$ and $1-(-1)^{k} \geq 0$ for all $k$, and $2^{k}-2-2 k-2 k(k-1)=2^{k}-2-2 k^{2} \geq 0$ for $k \geq 7$. Moreover,

$$
2^{k}-2-2 k^{2}+3(k-1)\left(1+(-1)^{k}\right)+2 k\left(1-(-1)^{k}\right)= \begin{cases}0 & \text { for } k=4 \\ 0 & \text { for } k=5 \\ 20 & \text { for } k=6\end{cases}
$$

This shows that Equation (40) is satisfied for $t \geq 0$. Thus, when $F$ is a truncated exponential with negative rate, i.e. case (i),

$$
\sup _{x \leq y \leq z}\left\{\begin{array}{c}
{[\bar{F}(y)-\bar{F}(z)][2 L(x, y)-R(x, y)]} \\
-[\bar{F}(x)-\bar{F}(y)] R(x, y)
\end{array}\right\} \leq 0
$$

Case (ii) can be analyzed similarly. In this case, using the same notation, $\Delta_{1}=\beta(y-x)$ and $\Delta_{2}=\beta(z-y)$, where $\beta$ is now the positive rate of the exponential, the constraint is tight and hence equivalent to

$$
\frac{1}{P_{1}\left(\Delta_{1}\right)}+\frac{1}{P_{1}\left(-\Delta_{2}\right)}=2
$$

We must show now that

$$
\frac{\left(e^{\Delta_{2}}-1\right)}{\left(1-e^{-\Delta_{1}}\right)}\left(2 P_{1}\left(-\Delta_{1}\right)-3 P_{2}\left(-\Delta_{1}\right)\right) \leq P_{2}\left(-\Delta_{1}\right) .
$$

Now, $\Delta_{2} \leq \Delta_{1}$ and this implies that $\left(e^{\Delta_{2}}-1\right) /\left(1-e^{-\Delta_{1}}\right) \leq P_{2}\left(\Delta_{1}\right) / P_{2}\left(-\Delta_{1}\right)$. Hence, we must show that for all $t \geq 0$,

$$
P_{2}(t)\left(2 P_{1}(-t)-3 P_{2}(-t)\right) \leq P_{2}(-t)^{2}
$$

or equivalently, using that $P_{2}(-t) e^{t}=P_{1}(t)-P_{2}(t)$,

$$
t^{4} e^{t} P_{2}(t)\left(3 P_{2}(t)-P_{1}(t)\right) \leq t^{4}\left(P_{1}(t)-P_{2}(t)\right)^{2}
$$


Since

$$
\begin{gathered}
t^{4} P_{2}(t)^{2}=\sum_{k=4}^{\infty} \frac{t^{k}}{k !}\left[2^{k}-2-2 k\right], \\
t^{4} e^{t} P_{2}(t)^{2}=\sum_{k=4}^{\infty} \frac{t^{k}}{k !}\left[3^{k}-2^{k+1}-k 2^{k}+1+2 k+k(k-1)\right], \\
t^{4} P_{2}(t) P_{1}(t)=\sum_{k=4}^{\infty} \frac{t^{k}}{k !}\left[k 2^{k-1}-2 k-k(k-1)\right], \\
t^{4} e^{t} P_{2}(t) P_{1}(t)=\sum_{k=4}^{\infty} \frac{t^{k}}{k !}\left[k 3^{k-1}-k 2^{k}-k(k-1) 2^{k-2}+k+k(k-1)\right], \\
t^{4} P_{1}(t)^{2}=\sum_{k=4}^{\infty} \frac{t^{k}}{k !}\left[k(k-1) 2^{k-2}-2 k(k-1)\right],
\end{gathered}
$$

we must show that

$$
\sum_{k=4}^{\infty} \frac{t^{k}}{k !}\left[\begin{array}{l}
k(k-1) \cdot 2^{k-2}-2 k(k-1) \\
-2 k \cdot 2^{k-1}+4 k+2 k(k-1) \\
+2^{k}-2-2 k \\
+k \cdot 3^{k-1}-k \cdot 2^{k}-k(k-1) \cdot 2^{k-2}+k+k(k-1) \\
-3^{k+1}+3 \cdot 2^{k+1}+3 k \cdot 2^{k}-3-6 k-3 k(k-1)
\end{array}\right] \geq 0 .
$$

The coefficients in the brackets are equal to

$$
(k-9) 3^{k-1}+(k+7) 2^{k}+\left(-2 k^{2}-k-5\right)
$$

They are clearly non-negative for $k \geq 9$. For smaller values, we have

$$
(k-9) 3^{k-1}+(k+7) 2^{k}+\left(-2 k^{2}-k-5\right)= \begin{cases}0 & \text { for } k=4, \\ 0 & \text { for } k=5, \\ 20 & \text { for } k=6, \\ 224 & \text { for } k=7 \\ 1512 & \text { for } k=8 .\end{cases}
$$

Hence, for all $t \geq 0$,

$$
t^{4} e^{t} P_{2}(t)\left(3 P_{2}(t)-P_{1}(t)\right) \leq t^{4}\left(P_{1}(t)-P_{2}(t)\right)^{2},
$$

and thus, when $F$ is a truncated exponential with positive rate, i.e. case (ii),

$$
\sup _{x \leq y \leq z}\left\{\begin{array}{c}
{[\bar{F}(y)-\bar{F}(z)][2 L(x, y)-R(x, y)]} \\
-[\bar{F}(x)-\bar{F}(y)] R(x, y)
\end{array}\right\} \leq 0
$$

Case (iii) is straightforward. When the distribution is uniform, $L(x, y)=R(x, y)=(y-x) / 2$ for all $x \leq y$. Also the condition $L(x, y) \geq R(y, z)$ is equivalent to $z-y \leq y-x$. Thus,

$$
[\bar{F}(y)-\bar{F}(z)][2 L(x, y)-R(x, y)]-[\bar{F}(x)-\bar{F}(y)] R(x, y)=\frac{1}{2}(y-x)(z+x-2 y) \leq 0 .
$$




\section{Theorem 6}

Proof. Supplier $i$ is active in this equilibrium. Since the demand follows a border distribution, supplier $i$ bids in the boundary of some region $A_{l h}$ constructed with all the bids except $i$ 's. If the bid $\left(w_{i}, v_{i}\right)$ belongs to more than one region, choose $A_{l h}$ with $l$ and $h$ active. We must consider two cases, either there is no supplier in the lower envelope between the bids of $l$ and $h$, or there is one.

In the first case, there is $j, j$ being $l$ or $h$, such that $j$ is active, and $\left(w_{i}, v_{i}\right)=\left(w_{j}, v_{j}\right)$, from Theorem 2. From Proposition $2,\left(w_{i}, v_{i}\right)$ belongs in the segment $\left[\left(c_{i}, f_{i}\right) ;\left(c_{j}, f_{j}\right)\right]$.

In the second case, there is one supplier, $k$ on the lower envelope between $l$ and $h$ such that the bid $\left(w_{i}, v_{i}\right)$ is in the border of $A_{l h}$ and $A_{l k}$ or $A_{l h}$ and $A_{k h} . k$ is thus inactive because of supplier $i$, and either the bids of $l, k$ and $i$ are aligned, or those of $i, k$ and $h$. Such a situation is depicted in Figure 8. Hence, we find $j, j$ being $l$ or $h$, active, such that $\left(w_{i}, v_{i}\right)$ is equal to $\left(w_{j}, v_{j}\right)+\theta\left(w_{k}-w_{j}, v_{k}-v_{j}\right)$ for some non-negative $\theta$.

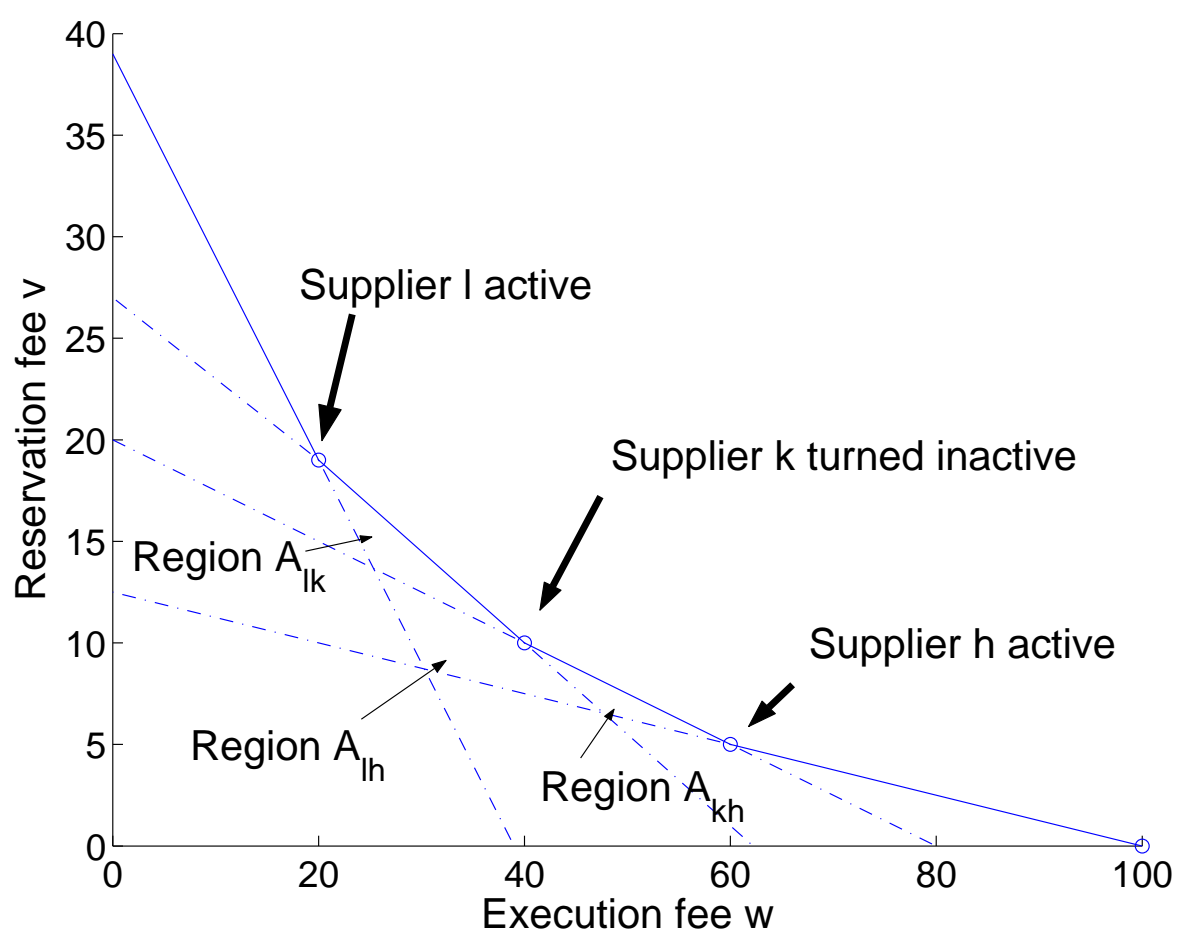

Figure 8: Suppliers $l$ and $h$ are active and supplier $k$ is turned inactive by supplier $i$ 's bid. 
